Pacific

Journal of

Mathematics

\title{
ON DEMAZURE AND LOCAL WEYL MODULES FOR AFFINE HYPERALGEBRAS
}

Angelo Bianchi, Tiago Macedo and Adriano Moura 


\title{
ON DEMAZURE AND LOCAL WEYL MODULES FOR AFFINE HYPERALGEBRAS
}

\author{
Angelo Bianchi, Tiago Macedo and Adriano Moura
}

\begin{abstract}
We establish the existence of Demazure flags for graded local Weyl modules for hyper current algebras in positive characteristic. If the underlying simple Lie algebra is simply laced, the flag has length one; that is, the graded local Weyl modules are isomorphic to Demazure modules. This extends to the positive characteristic setting results of Chari and Loktev, Fourier and Littelmann, and Naoi for current algebras in characteristic zero. Using this result, we prove that the character of local Weyl modules for hyper loop algebras depend only on the highest weight, but not on the (algebraically closed) ground field, and deduce a tensor product factorization for them.
\end{abstract}

\section{Introduction}

Let $\mathfrak{g}$ be a semisimple finite-dimensional Lie algebra over the complex numbers and, given an algebraically closed field $\mathbb{F}$, let $G_{\mathbb{E}}$ be a connected, simply connected, semisimple algebraic group over $\mathbb{F}$ of the same Lie type as $\mathfrak{g}$. The category of finite-dimensional $G_{\mathbb{F}}$-modules is equivalent to that of the hyperalgebra $U_{\mathbb{F}}(\mathfrak{g})$. The hyperalgebra is a Hopf algebra obtained from the universal enveloping algebra of $\mathfrak{g}$ by first choosing a certain integral form and then changing scalars to $\mathbb{F}$ (this process is often referred to as reduction modulo $p$ ). If the characteristic of $\mathbb{F}$ is positive, the category of finite-dimensional $G_{\mathbb{F}}$-modules is not semisimple, and the modules obtained by reduction modulo $p$ of simple g-modules - called Weyl modules provide examples of indecomposable, reducible modules. The Weyl modules have several interesting properties which are independent of $\mathbb{F}$ such as: a description in terms of generators and relations, being the universal highest-weight modules of the category of finite-dimensional $G_{\mathbb{F}}$-modules, their characters are given by the Weyl character formula.

Consider now the loop algebra $\tilde{\mathfrak{g}}=\mathfrak{g} \otimes \mathbb{C}\left[t, t^{-1}\right]$. The finite-dimensional representation theory of $\tilde{\mathfrak{g}}$ was initiated by Chari and Presley [1986], where the

Partially supported by FAPESP grants 2011/22322-4 (Bianchi) and 2009/05887-8 (Macedo), and CNPq grant 303667/2011-7 (Moura).

MSC2010: primary 17B10, 17B65; secondary 17B37, $20 \mathrm{G} 42$.

Keywords: Weyl modules, Demazure modules, hyperalgebras, affine Kac-Moody algebras, twisted affine algebras. 
simple modules were classified in terms of tensor products of evaluation modules. Differently from the category of finite-dimensional $\mathfrak{g}$-modules, the category of finite-dimensional $\tilde{\mathfrak{g}}$-modules is not semisimple. Therefore, it is natural to ask if there is a notion analogue to that of Weyl modules for $\tilde{\mathfrak{g}}$. Chari and Presley [2001] proved that the simple finite-dimensional $\tilde{\mathfrak{g}}$-modules are highest-weight in an appropriate sense and introduced the Weyl modules for $\tilde{\mathfrak{g}}$ in terms of generator and relations which are the natural analogues of the relations for the original Weyl modules. The highest-weight vector is now an eigenvector for the action of the loop algebra $\tilde{\mathfrak{h}}$ over the Cartan subalgebra $\mathfrak{h}$ of $\mathfrak{g}$. Because of this, it eventually became common practice to use the terms $\ell$-weight and highest- $\ell$-weight. In particular, it was shown in [Chari and Pressley 2001] that the just-introduced Weyl modules share a second property with their older relatives: they are the universal finite-dimensional highest- $\ell$-weight modules. These results were immediately quantized and, still in the same paper, the notion of Weyl modules for the quantum loop algebra $U_{q}(\tilde{\mathfrak{g}})$ was introduced. Chari and Presley conjectured (and proved for $\mathfrak{g}=\mathfrak{s l}_{2}$ ) that the Weyl modules for $\tilde{\mathfrak{g}}$ were classical limits of quantum Weyl modules. Moreover, all Weyl modules for $\tilde{\mathfrak{g}}$ could be obtained as classical limits of quantum Weyl modules which are actually irreducible. This can be viewed as the analogue of the property that the original Weyl modules are obtained by reduction modulo $p$ from simple $\mathfrak{g}$-modules.

Motivated by bringing the discussion of the last paragraph to the positive characteristic setting, [Jakelić and Moura 2007] initiated the study of the finite-dimensional representation theory of the hyperalgebras associated to $\tilde{\mathfrak{g}}$, which we refer to as hyper loop algebras. Several basic properties of the underlying abelian category were established and, in particular, the notion of Weyl modules was introduced. Moreover, it was shown that certain Weyl modules for $\tilde{\mathfrak{g}}$ can be reduced modulo $p$. In analogy with the previous paragraphs, it is natural to conjecture that the reduction modulo $p$ of a Weyl module is again a Weyl module (the difference is that now we cannot restrict attention to Weyl modules which are irreducible since there are too few of these).

In the meantime, two partial proofs of Chari and Presley's conjecture appeared [Chari and Loktev 2006; Fourier and Littelmann 2007]. Namely, it follows from a tensor product factorization of the Weyl modules for $\tilde{\mathfrak{g}}$ proved in [Chari and Pressley 2001] together with the fact that the irreducible quantum Weyl modules are tensor products of fundamental modules, that it suffices to compute the dimension of graded analogues of Weyl modules for the current algebra $\mathfrak{g}[t]=\mathfrak{g} \otimes \mathbb{C}[t]$. These graded analogues of Weyl modules were introduced in [Feigin and Loktev 2004] as a particular case of a class of modules (named local Weyl modules) for algebras of the form $\mathfrak{g} \otimes A$, where $A$ is a commutative associative algebra (see also [Chari et al. 2010; Fourier et al. 2012] and references therein for more on the recent 
development of the representation theory of such algebras). For $\mathfrak{g}$ of type $A$, the dimensions of the graded Weyl modules were computed in [Chari and Loktev 2006] by explicitly exhibiting a vector space basis. As a consequence, it was observed that they are isomorphic to certain Demazure modules. For a general simply-laced Lie algebra, this isomorphism was proved in [Fourier and Littelmann 2007] by using a certain presentation of Demazure modules by generators and relations as well as by studying fusion products. In particular, the dimension of the graded Weyl modules could be computed resulting in a proof of the conjecture. It was also shown in [Fourier and Littelmann 2007] that such isomorphisms do not exist in general in the non-simply laced case. It was pointed out by Nakajima that the general case could be deduced by using global bases theory (this proof remains unpublished, but a brief sketch is given in the introduction of [Fourier and Littelmann 2007]). The relation with Demazure modules in the nonsimply laced case was finally established in completely generality in [Naoi 2012] where it was shown that the graded Weyl modules for $\mathfrak{g}[t]$ admit Demazure flags, that is, filtrations whose quotients are Demazure modules. Such flags are actually obtained from results of Joseph [2003; 2006] (see also [Littelmann 1998]) on global bases for tensor products of Demazure modules. Therefore, in the nonsimply laced case, the relation between Weyl and Demazure modules is, so far, dependent on the theory of global bases, although in a different manner than Nakajima's proposed proof.

The goal of the present paper is to extend to the positive characteristic context the results of [Fourier and Littelmann 2007; Naoi 2012] and prove the conjecture of [Jakelić and Moura 2007] on reduction modulo $p$ of Weyl modules for hyper loop algebras. Moreover, we prove a tensor product factorization of Weyl modules the hyperalgebraic analogue of that proved in [Chari and Pressley 2001]. However, due to the extra technical difficulties which arise when dealing with hyperalgebras in positive characteristic, there are several differences in our proofs from those used in the characteristic zero setting. For instance, the tensor product factorization was originally used to restrict the study to computing the dimension of the graded Weyl modules for current algebras. In the positive characteristic setting, we actually deduce the tensor product factorization from the computation of the dimension. Also, for proving the existence of the Demazure flags, some arguments used in [Naoi 2012] do not admit a hyperalgebraic analogue. Our approach to overcome these issues actually makes use of the characteristic-zero version of the same statements. We also use the fact proved in [Mathieu 1988; 1989] that the characters of Demazure modules do not depend on the ground field. Different presentations of Demazure modules in terms of generator and relations are needed for different parts of the argument. For $\mathfrak{g}$ of type $G_{2}$, technical issues for proving one of these presentations require that we restrict ourselves to characteristic different than 2 and 3. Outside type $G_{2}$, there is no restriction in the characteristic of the ground field. 
While this paper was being finished, new ideas for studying Demazure, local Weyl modules, and Kirillov-Reshetikhin modules are introduced. In particular, several results of [Chari and Pressley 2001; Fourier and Littelmann 2007; Naoi 2012] are recovered and generalized. Moreover, new (and simpler) presentations in terms of generators and relations for Demazure modules are obtained. It will be interesting to study if the ideas and results of [Chari and Venkatesh 2014] can be brought to the positive characteristic setting as well.

The paper is organized as follows. We start Section 1 fixing the notation regarding finite and affine types, Kac-Moody algebras and reviewing the construction of the hyperalgebras. Next, using generators and relations, we define the Weyl modules for hyper loop algebras, their graded analogues for hyper current algebras, and the subclass of the class of Demazure modules which is relevant for us. We then state our main result (Theorem 1.5.2) and recall the precise statement (1.5.4) of the conjecture in [Jakelić and Moura 2007]. Theorem 1.5.2 is stated in 4 parts. Part (a) states the isomorphism between graded Weyl modules and Demazure modules for simply laced $\mathfrak{g}$. Part (b) states the existence of Demazure flags for graded Weyl modules. Part (c) establishes an isomorphism between a given graded Weyl module and a twist of certain Weyl module for the hyper loop algebra. Finally, part (d) is the aforementioned tensor product factorization. In Section 2, we fix some further notation and establish a few technical results needed in the proofs.

Section 3.1 brings a review of the finite-dimensional representation theory of the finite-type hyperalgebras while Section 3.2 gives a very brief account of the relevant results from [Jakelić and Moura 2007]. Section 3.3 is concerned with the category of finite-dimensional graded modules for the hyper current algebras. The main results of this subsection are Theorem 3.3.4, where the basic properties of the category are established, and Corollary 3.3.3 which states that the graded Weyl modules for $\mathfrak{g}[t]$ admit integral forms. Assuming Theorem 1.5.2(b), we prove (1.5.4) in Section 3.4. The proof actually makes use of the characteristic-zero version of all parts of Theorem 1.5.2 as well as [Naoi 2012, Corollary A] (stated here as Proposition 3.4.1). In Section 3.5, we prove a second presentation of Demazure modules in terms of generator and relations. It basically replaces a highest-weight generator by a lowest-weight one. This is the presentation which allows us to use the results of [Mathieu 1988; 1989] on the independence of the characters of Demazure modules on the ground field.

In the first three subsections of Section 4 we collect the results of [Joseph 2003; 2006] on crystal and global bases which we need to prove Theorem 4.4.1 which is an integral analogue of [Naoi 2012, Corollary 4.16] on the existence of higher level Demazure flags for Demazure modules when the underlying simple Lie algebra $\mathfrak{g}$ is simply laced. We remark that the proof of Theorem 4.4.1 is the only one where the theory of global bases is used. We further remark that, in order to prove 
Theorem 1.5.2(b), we only need the statement of Theorem 4.4.1 for $\mathfrak{g}$ of type $A$. We observe that the only other place quantum groups are being used here is in the proof of the characteristic-zero version of Theorem 1.5.2(c); [Fourier and Littelmann 2007, Lemmas 1 and 3 and Equation (15)].

Theorem 1.5.2 is proved in Section 5. In particular, in Section 5.2, we prove a positive characteristic analogue of [Naoi 2012, Proposition 4.1] which is a third presentation of Demazure modules in terms of generator and relations in the case that $\mathfrak{g}$ is not simply laced. This is where the restriction on the characteristic of the ground field for type $G_{2}$ appears. Parts (b) and (c) of Theorem 1.5.2 are proved in Sections 5.3 and 5.4, respectively. Finally, in Sections 5.5 and 5.6, we prove that the tensor product of finite-dimensional highest- $\ell$-weight modules for hyper loop algebras with relatively prime highest $\ell$-weights is itself a highest- $\ell$-weight module and deduce Theorem 1.5.2(d). As an application of Theorem 1.5.2, we end the paper proving that the graded Weyl modules are fusion products of Weyl modules with "smaller" highest weights (Proposition 5.7.1).

\section{The main results}

1.1. Finite-type data. Let $\mathfrak{g}$ be a finite-dimensional simple Lie algebra over $\mathbb{C}$ with a fixed Cartan subalgebra $\mathfrak{h} \subset \mathfrak{g}$. The associated root system will be denoted by $R \subset \mathfrak{h}^{*}$. We fix a simple system $\Delta=\left\{\alpha_{i}: i \in I\right\} \subset R$ and denote the corresponding set of positive roots by $R^{+}$. The Borel subalgebra associated to $R^{+}$will be denoted by $\mathfrak{b}^{+} \subset \mathfrak{g}$ and the opposite Borel subalgebra will be denoted by $\mathfrak{b}^{-} \subset \mathfrak{g}$. We fix a Chevalley basis of the Lie algebra $\mathfrak{g}$ consisting of $x_{\alpha}^{ \pm} \in \mathfrak{g}_{ \pm \alpha}$, for each $\alpha \in R^{+}$, and $h_{i} \in \mathfrak{h}$, for each $i \in I$. We also define $h_{\alpha} \in \mathfrak{h}, \alpha \in R^{+}$, by $h_{\alpha}=\left[x_{\alpha}^{+}, x_{\alpha}^{-}\right]$ (in particular, $h_{i}=h_{\alpha_{i}}, i \in I$ ) and set $R^{\vee}=\left\{h_{\alpha} \in \mathfrak{h}: \alpha \in R\right\}$. We often simplify notation and write $x_{i}^{ \pm}$in place of $x_{\alpha_{i}}^{ \pm}, i \in I$. Let (, ) denote the invariant symmetric bilinear form on $\mathfrak{g}$ such that $\left(h_{\theta}, h_{\theta}\right)=2$, where $\theta$ is the highest root of $\mathfrak{g}$. Let $v: \mathfrak{h} \rightarrow \mathfrak{h}^{*}$ be the linear isomorphism induced by (, ) and keep denoting by (, ) the nondegenerate bilinear form induced by $v$ on $\mathfrak{h}^{*}$. Notice that

$$
\left(x_{\alpha}^{+}, x_{\alpha}^{-}\right)=\frac{2}{(\alpha, \alpha)} \text { for all } \alpha \in R^{+}
$$

and

$$
(\alpha, \alpha)= \begin{cases}2 & \text { if } \alpha \text { is long, } \\ 2 / r^{\vee} & \text { if } \alpha \text { is short, }\end{cases}
$$

where $r^{\vee} \in\{1,2,3\}$ is the lacing number of $\mathfrak{g}$. For notational convenience, set

$$
r_{\alpha}^{\vee}=\frac{2}{(\alpha, \alpha)}= \begin{cases}1, & \text { if } \alpha \text { is long, } \\ r^{\vee}, & \text { if } \alpha \text { is short. }\end{cases}
$$


We shall need the following fact [Carter 1972, Section 4.2]. Given $\alpha \in R$, let $x_{\alpha}=x_{ \pm \alpha}^{ \pm}$according to whether $\alpha \in \pm R^{+}$. For $\alpha, \beta \in R$ let $p=\max \{n: \beta-n \alpha \in R\}$. Then there exists $\varepsilon \in\{-1,1\}$ such that

$$
\left[x_{\alpha}, x_{\beta}\right]=\varepsilon(p+1) x_{\alpha+\beta} .
$$

We define the weight lattice $P=\left\{\lambda \in \mathfrak{h}^{*}: \lambda\left(h_{\alpha}\right) \in \mathbb{Z}\right.$ for all $\left.\alpha \in R\right\}$, the subset of dominant weights $P^{+}=\left\{\lambda \in P: \lambda\left(h_{\alpha}\right) \in \mathbb{N}\right.$ for all $\left.\alpha \in R^{+}\right\}$, the coweight lattice $P^{\vee}=\{h \in \mathfrak{h}: \alpha(h) \in \mathbb{Z}$ for all $\alpha \in R\}$, and the subset of dominant coweights $P^{\vee+}=\left\{h \in P^{\vee}: \alpha(h) \in \mathbb{N}\right.$ for all $\left.\alpha \in R^{+}\right\}$. We denote the fundamental weights by $\omega_{i}, i \in I$, the root lattice of $\mathfrak{g}$ by $Q$, and we let $Q^{+}=\mathbb{Z}_{\geq 0} R^{+}$. We consider the usual partial order on $\mathfrak{h}^{*}: \mu \leq \lambda$ if and only if $\lambda-\mu \in Q^{+}$. The Weyl group $\mathcal{W}$ of $\mathfrak{g}$ is the subgroup of $\operatorname{Aut}_{\mathbb{C}}\left(\mathfrak{h}^{*}\right)$ generated by the simple reflections $s_{i}, i \in I$, defined by $s_{i}(\mu)=\mu-\mu\left(h_{i}\right) \alpha_{i}$ for all $\mu \in \mathfrak{h}^{*}$. As usual, $w_{0}$ denotes the longest element in $\mathcal{W}$.

1.2. Affine-type data. Consider the loop algebra $\tilde{\mathfrak{g}}=\mathfrak{g} \otimes \mathbb{C}\left[t, t^{-1}\right]$, with Lie bracket given by $\left[x \otimes t^{r}, y \otimes t^{s}\right]=[x, y] \otimes t^{r+s}$, for any $x, y \in \mathfrak{g}, r, s \in \mathbb{Z}$. We identify $\mathfrak{g}$ with the subalgebra $\mathfrak{g} \otimes 1$ of $\mathfrak{\mathfrak { g }}$. The subalgebra $\mathfrak{g}[t]=\mathfrak{g} \otimes \mathbb{C}[t]$ is the current algebra of $\mathfrak{g}$. If $\mathfrak{a}$ is a subalgebra of $\mathfrak{g}$, let $\tilde{\mathfrak{a}}=\mathfrak{a} \otimes \mathbb{C}\left[t, t^{-1}\right]$ and $\mathfrak{a}[t]=\mathfrak{a} \otimes \mathbb{C}[t]$. Let also $\mathfrak{a}[t]_{ \pm}:=\mathfrak{a} \otimes\left(t^{ \pm 1} \mathbb{C}\left[t^{ \pm 1}\right]\right)$. In particular, as vector spaces,

$$
\tilde{\mathfrak{g}}=\tilde{\mathfrak{n}}^{-} \oplus \tilde{\mathfrak{h}} \oplus \tilde{\mathfrak{n}}^{+} \quad \text { and } \quad \mathfrak{g}[t]=\mathfrak{n}^{-}[t] \oplus \mathfrak{h}[t] \oplus \mathfrak{n}^{+}[t] .
$$

The affine Kac-Moody algebra $\hat{\mathfrak{g}}$ is the 2-dimensional extension $\hat{\mathfrak{g}}:=\tilde{\mathfrak{g}} \oplus \mathbb{C} c \oplus \mathbb{C} d$ of $\tilde{\mathfrak{g}}$ with Lie bracket given by

$$
\begin{gathered}
{\left[x \otimes t^{r}, y \otimes t^{s}\right]=[x, y] \otimes t^{r+s}+r \delta_{r,-s}(x, y) c,} \\
{[c, \hat{\mathfrak{g}}]=\{0\}, \quad \text { and } \quad\left[d, x \otimes t^{r}\right]=r x \otimes t^{r}}
\end{gathered}
$$

for any $x, y \in \mathfrak{g}, r, s \in \mathbb{Z}$. Observe that if $\hat{\mathfrak{g}}^{\prime}=[\hat{\mathfrak{g}}, \hat{\mathfrak{g}}]$ is the derived subalgebra of $\hat{\mathfrak{g}}$, then $\hat{\mathfrak{g}}^{\prime}=\tilde{\mathfrak{g}} \oplus \mathbb{C} c$, and we have a nonsplit short exact sequence of Lie algebras $0 \rightarrow \mathbb{C} c \rightarrow \hat{\mathfrak{g}}^{\prime} \rightarrow \tilde{\mathfrak{g}} \rightarrow 0$.

Set $\hat{\mathfrak{h}}^{\prime}=\mathfrak{h} \oplus \mathbb{C} c$. Notice that $\mathfrak{g}, \mathfrak{g}[t]$, and $\mathfrak{g}[t]_{ \pm}$remain subalgebras of $\hat{\mathfrak{g}}$. Set

$$
\hat{\mathfrak{h}}=\mathfrak{h} \oplus \mathbb{C} c \oplus \mathbb{C} d, \quad \hat{\mathfrak{n}}^{ \pm}=\mathfrak{n}^{ \pm} \oplus \mathfrak{g}[t]_{ \pm}, \quad \text { and } \quad \hat{\mathfrak{b}}^{ \pm}=\hat{\mathfrak{n}}^{ \pm} \oplus \hat{\mathfrak{h}} .
$$

The root system, positive root system, and set of simple roots associated to the triangular decomposition $\hat{\mathfrak{g}}=\hat{\mathfrak{n}}^{-} \oplus \hat{\mathfrak{h}} \oplus \hat{\mathfrak{n}}^{+}$will be denoted by $\hat{R}, \hat{R}^{+}$and $\hat{\Delta}$, respectively. Let $\hat{I}=I \sqcup\{0\}$ and $h_{0}=c-h_{\theta}$, so that $\left\{h_{i}: i \in \hat{I}\right\} \cup\{d\}$ is a basis of $\hat{\mathfrak{h}}$. Identify $\mathfrak{h}^{*}$ with the subspace $\left\{\lambda \in \hat{\mathfrak{h}}^{*}: \lambda(c)=\lambda(d)=0\right\}$. Let also $\delta \in \hat{\mathfrak{h}}^{*}$ be such that $\delta(d)=1$ and $\delta\left(h_{i}\right)=0$ for all $i \in \hat{I}$ and define $\alpha_{0}=\delta-\theta$. Then $\hat{\Delta}=\left\{\alpha_{i}: i \in \hat{I}\right\}, \hat{R}^{+}=R^{+} \cup\left\{\alpha+r \delta: \alpha \in R \cup\{0\}, r \in \mathbb{Z}_{>0}\right\}$, and $\hat{\mathfrak{g}}_{\alpha+r \delta}=\mathfrak{g}_{\alpha} \otimes t^{r}$ if $\alpha \in R, r \in \mathbb{Z}$, and $\hat{\mathfrak{g}}_{r \delta}=\mathfrak{h} \otimes t^{r}$, if $r \in \mathbb{Z} \backslash\{0\}$. Observe that

$$
\alpha(c)=0 \text { for all } \alpha \in \hat{R} \text {. }
$$


A root $\gamma \in \hat{R}$ is called real if $\gamma=(\alpha+r \delta)$ with $\alpha \in R, r \in \mathbb{Z}$, and imaginary if $\gamma=r \delta$ with $r \in \mathbb{Z} \backslash\{0\}$. Set $x_{\alpha, r}^{ \pm}=x_{\alpha}^{ \pm} \otimes t^{r}, h_{\alpha, r}=h_{\alpha} \otimes t^{r}, \alpha \in R^{+}, r \in \mathbb{Z}$. We often simplify notation and write $x_{i, r}^{ \pm}$and $h_{i, r}$ in place of $x_{\alpha_{i}, r}^{ \pm}$and $h_{\alpha_{i}, r}, i \in I, r \in \mathbb{Z}$. Observe that $\left\{x_{\alpha, r}^{ \pm}, h_{i, r}: \alpha \in R^{+}, i \in I, r \in \mathbb{Z}\right\}$ is a basis of $\tilde{\mathfrak{g}}$. Given $\alpha \in R^{+}$and $r \in \mathbb{Z}_{>0}$, set

$$
x_{ \pm \alpha+r \delta}^{+}=x_{\alpha, r}^{ \pm}, \quad x_{ \pm \alpha+r \delta}^{-}=x_{\alpha,-r}^{\mp}, \quad h_{ \pm \alpha+r \delta}=\left[x_{ \pm \alpha+r \delta}^{+}, x_{ \pm \alpha+r \delta}^{-}\right]= \pm h_{\alpha}+r r_{\alpha}^{\vee} c .
$$

Define also $\Lambda_{i} \in \hat{\mathfrak{h}}^{*}, i \in \hat{I}$, by the requirement $\Lambda_{i}(d)=0, \Lambda_{i}\left(h_{j}\right)=\delta_{i j}$ for all $i, j \in \hat{I}$. Set $\hat{P}=\mathbb{Z} \delta \oplus \bigoplus_{i \in \hat{I}} \mathbb{Z} \Lambda_{i}, \hat{P}^{+}=\mathbb{Z} \delta \oplus \bigoplus_{i \in \hat{I}} \mathbb{N} \Lambda_{i}, \hat{P}^{\prime}=\bigoplus_{i \in \hat{I}} \mathbb{Z} \Lambda_{i}$, and $\hat{P}^{\prime+}=\hat{P}^{\prime} \cap \hat{P}^{+}$. Notice that

$$
\Lambda_{0}(h)=0 \quad \Longleftrightarrow \quad h \in \mathfrak{h} \oplus \mathbb{C} d \text { and } \Lambda_{i}-\omega_{i}=\omega_{i}\left(h_{\theta}\right) \Lambda_{0} \text { for all } i \in I .
$$

Hence, $\hat{P}=\mathbb{Z} \Lambda_{0} \oplus P \oplus \mathbb{Z} \delta$. Given $\Lambda \in \hat{P}$, the number $\Lambda(c)$ is called the level of $\Lambda$. By (1.2.1), the level of $\Lambda$ depends only on its class modulo the root lattice $\hat{Q}$. Set also $\hat{Q}^{+}=\mathbb{Z}_{\geq 0} \hat{R}^{+}$and let $\widehat{W}$ denote the affine Weyl group, which is generated by the simple reflections $s_{i}, i \in \hat{I}$. Finally, observe that $\left\{\Lambda_{0}, \delta\right\} \cup \Delta$ is a basis of $\hat{\mathfrak{h}}^{*}$.

1.3. Integral forms and hyperalgebras. We use the following notation. Given a $\mathbb{Q}$-algebra $U$ with unity, an element $x \in U$, and $k \in \mathbb{N}$, set

$$
x^{(k)}=\frac{1}{k !} x^{k} \quad \text { and } \quad\left(\begin{array}{l}
x \\
k
\end{array}\right)=\frac{1}{k !} x(x-1) \cdots(x-k+1) .
$$

In the case $U=U(\tilde{\mathfrak{g}})$, we also introduce elements $\Lambda_{x, \pm r} \in U(\tilde{\mathfrak{g}}), x \in \mathfrak{g}, r \in \mathbb{N}$, by the following identity of power series in the variable $u$ :

$$
\Lambda_{x}^{ \pm}(u):=\sum_{r \geq 0} \Lambda_{x, \pm r} u^{r}=\exp \left(-\sum_{s>0} \frac{x \otimes t^{ \pm s}}{s} u^{s}\right) .
$$

Most of the time we will work with such elements with $x=h_{\alpha}$ for some $\alpha \in R^{+}$. We then simplify notation and write $\Lambda_{\alpha}^{ \pm}(u)=\Lambda_{h_{\alpha}}^{ \pm}(u)$, and if $\alpha=\alpha_{i}$ for some $i \in I$, we simply write $\Lambda_{i}^{ \pm}(u)=\Lambda_{h_{i}}^{ \pm}(u)$. To shorten notation, we also set $\Lambda_{x}(u)=\Lambda_{x}^{+}(u)$.

Consider the $\mathbb{Z}$-subalgebra $U_{\mathbb{Z}}\left(\hat{\mathfrak{g}}^{\prime}\right)$ of $U\left(\hat{\mathfrak{g}}^{\prime}\right)$ generated by the set

$$
\left\{\left(x_{\alpha, r}^{ \pm}\right)^{(k)}: \alpha \in R^{+}, r \in \mathbb{Z}, k \in \mathbb{N}\right\} .
$$

By [Garland 1978, Theorem 5.8], it is a free $\mathbb{Z}$-submodule of $U\left(\hat{\mathfrak{g}}^{\prime}\right)$ and satisfies $\mathbb{C} \otimes_{\mathbb{Z}} U_{\mathbb{Z}}\left(\hat{\mathfrak{g}}^{\prime}\right)=U\left(\hat{\mathfrak{g}}^{\prime}\right)$. In other words, $U_{\mathbb{Z}}\left(\hat{\mathfrak{g}}^{\prime}\right)$ is an integral form of $U\left(\hat{\mathfrak{g}}^{\prime}\right)$. Moreover, the image of $U_{\mathbb{Z}}\left(\hat{\mathfrak{g}}^{\prime}\right)$ in $U(\tilde{\mathfrak{g}})$ is an integral form of $U(\tilde{\mathfrak{g}})$ denoted by $U_{\mathbb{Z}}(\tilde{\mathfrak{g}})$. For a Lie subalgebra $\mathfrak{a}$ of $\hat{\mathfrak{g}}^{\prime}$ set

$$
U_{\mathbb{Z}}(\mathfrak{a})=U(\mathfrak{a}) \cap U_{\mathbb{Z}}\left(\hat{\mathfrak{g}}^{\prime}\right),
$$


and similarly for subalgebras of $\tilde{\mathfrak{g}}$. The subalgebra $U_{\mathbb{Z}}(\mathfrak{g})$ coincides with the $\mathbb{Z}$ subalgebra of $U(\hat{\mathfrak{g}})$ generated by $\left\{\left(x_{\alpha}^{ \pm}\right)^{(k)}: \alpha \in R^{+}, k \in \mathbb{N}\right\}$. The subalgebra $U_{\mathbb{Z}}\left(\mathfrak{n}^{ \pm}\right)$ of $U_{\mathbb{Z}}(\mathfrak{g})$ is generated, as a $\mathbb{Z}$-subalgebra, by the set $\left\{\left(x_{\alpha}^{ \pm}\right)^{(k)}: \alpha \in R^{+}, k \in \mathbb{N}\right\}$ while $U_{\mathbb{Z}}(\mathfrak{h})$ is generated, as a $\mathbb{Z}$-subalgebra, by $\left\{\left(\begin{array}{c}h_{i} \\ k\end{array}\right): i \in I, k \in \mathbb{N}\right\}$. Similarly, the subalgebra $U_{\mathbb{Z}}\left(\mathfrak{n}^{ \pm}[t]\right)$ of $U_{\mathbb{Z}}(\mathfrak{g}[t])$ is generated, as a $\mathbb{Z}$-subalgebra, by the set $\left\{\left(x_{\alpha, r}^{ \pm}\right)^{(k)}: \alpha \in R^{+}, k \in \mathbb{N}, r \in \mathbb{Z}_{\geq 0}\right\}$ while $U_{\mathbb{Z}}\left(\mathfrak{h}[t]_{+}\right)$is generated by $\left\{\Lambda_{i, r}: i \in\right.$ $\left.I, r \in \mathbb{Z}_{>0}\right\}$. In fact, the latter is free commutative over the given set. The PoincaréBirkhoff-Witt (PBW) theorem implies that multiplication establishes isomorphisms of $\mathbb{Z}$-modules

$$
\begin{aligned}
U_{\mathbb{Z}}\left(\hat{\mathfrak{g}}^{\prime}\right) & \cong U_{\mathbb{Z}}\left(\hat{\mathfrak{n}}^{-}\right) \otimes U_{\mathbb{Z}}\left(\hat{\mathfrak{h}}^{\prime}\right) \otimes U_{\mathbb{Z}}\left(\hat{\mathfrak{n}}^{+}\right), \\
U_{\mathbb{Z}}(\tilde{\mathfrak{g}}) & \cong U_{\mathbb{Z}}\left(\tilde{\mathfrak{n}}^{-}\right) \otimes U_{\mathbb{Z}}(\tilde{\mathfrak{h}}) \otimes U_{\mathbb{Z}}\left(\tilde{\mathfrak{n}}^{+}\right), \\
U_{\mathbb{Z}}(\mathfrak{g}[t]) & \cong U_{\mathbb{Z}}\left(\mathfrak{n}^{-}[t]\right) \otimes U_{\mathbb{Z}}(\mathfrak{h}[t]) \otimes U_{\mathbb{Z}}\left(\mathfrak{n}^{+}[t]\right) .
\end{aligned}
$$

Moreover, restricted to $U_{\mathbb{Z}}(\tilde{\mathfrak{h}})$ this gives rise to an isomorphism of $\mathbb{Z}$-algebras

$$
U_{\mathbb{Z}}(\tilde{\mathfrak{h}}) \cong U_{\mathbb{Z}}\left(\mathfrak{h}[t]_{-}\right) \otimes U_{\mathbb{Z}}(\mathfrak{h}) \otimes U_{\mathbb{Z}}\left(\mathfrak{h}[t]_{+}\right) .
$$

In general, it may not be true that $U_{\mathbb{Z}}(\mathfrak{a})$ is an integral form of $U(\mathfrak{a})$. However, if $\mathfrak{a}$ has a basis consisting of real root vectors, an elementary use of the PBW theorem implies that this is true. We shall make use of algebras of this form later on.

Given a field $\mathbb{F}$, define the $\mathbb{F}$-hyperalgebra of $\mathfrak{a}$ by $U_{\mathbb{F}}(\mathfrak{a})=\mathbb{F} \otimes_{\mathbb{Z}} U_{\mathbb{Z}}(\mathfrak{a})$, where $\mathfrak{a}$ is any of the Lie algebras with $\mathbb{Z}$-forms defined above. Clearly, if the characteristic of $\mathbb{F}$ is zero, the algebra $U_{\mathbb{F}}(\tilde{\mathfrak{g}})$ is naturally isomorphic to $U\left(\tilde{\mathfrak{g}}_{\mathbb{F}}\right)$ where $\tilde{\mathfrak{g}}_{\mathbb{F}}=\mathbb{F} \otimes_{\mathbb{Z}} \tilde{\mathfrak{g}}_{\mathbb{Z}}$ and $\tilde{\mathfrak{g}}_{\mathbb{Z}}$ is the $\mathbb{Z}$-span of the Chevalley basis of $\tilde{\mathfrak{g}}$, and similarly for all algebras $\mathfrak{a}$ we have considered. For fields of positive characteristic we just have an algebra homomorphism $U\left(\mathfrak{a}_{\mathbb{F}}\right) \rightarrow U_{\mathbb{F}}(\mathfrak{a})$ which is neither injective nor surjective. We will keep denoting by $x$ the image of an element $x \in U_{\mathbb{Z}}(\mathfrak{a})$ in $U_{\mathbb{F}}(\mathfrak{a})$. Notice that we have $U_{\mathbb{F}}(\tilde{\mathfrak{g}})=U_{\mathbb{F}}\left(\tilde{\mathfrak{n}}^{-}\right) U_{\mathbb{F}}(\tilde{\mathfrak{h}}) U_{\mathbb{F}}\left(\tilde{\mathfrak{n}}^{+}\right)$.

Given an algebraically closed field $\mathbb{F}$, let $\mathbb{A}$ be a Henselian discrete valuation ring of characteristic zero having $\mathbb{F}$ as its residue field. Set $U_{\mathbb{A}}(\mathfrak{a})=\mathbb{A} \otimes_{\mathbb{Z}} U_{\mathbb{Z}}(\mathfrak{a})$. Clearly $U_{\mathbb{F}}(\mathfrak{a}) \cong \mathbb{E} \otimes_{\mathbb{A}} U_{\mathbb{A}}(\mathfrak{a})$. We shall also fix an algebraic closure $\mathbb{K}$ of the field of fractions of $\mathbb{A}$. For an explanation why we shall need to move from integral forms to A-forms, see Remark 1.5.5 (and [Jakelić and Moura 2007, Section 4C]). As mentioned in the introduction, we assume the characteristic of $\mathbb{E}$ is either zero or at least 5 if $\mathfrak{g}$ is of type $G_{2}$.

Notice that the Hopf algebra structure of the universal enveloping algebras induce such structure on the hyperalgebras. For any Hopf algebra $H$, denote by $H^{0}$ its augmentation ideal.

1.4. The $\ell$-weight lattice. For a ring $A$, we shall denote by $A^{\times}$its set of unities. Consider the set $\mathscr{P}_{\mathbb{F}}^{+}$consisting of $|I|$-tuples $\omega=\left(\omega_{i}\right)_{i \in I}$, where $\omega_{i} \in \mathbb{E}[u]$ and 
$\omega_{i}(0)=1$ for all $i \in I$. Endowed with coordinatewise polynomial multiplication, $\mathscr{P}_{\mathbb{F}}^{+}$is a monoid. We denote by $\mathscr{P}_{\mathbb{F}}$ the multiplicative abelian group associated to $\mathscr{P}_{\mathbb{F}}^{+}$ which will be referred to as the $\ell$-weight lattice associated to $\mathfrak{g}$. One can describe $\mathscr{P}_{\mathbb{F}}$ in another way. Given $\mu \in P$ and $a \in \mathbb{F}^{\times}$, let $\omega_{\mu, a}$ be the element of $\mathscr{P}_{\mathbb{F}}$ defined as

$$
\left(\boldsymbol{\omega}_{\mu, a}\right)_{i}(u)=(1-a u)^{\mu\left(h_{i}\right)} \text { for all } i \in I .
$$

If $\mu=\omega_{i}$ is a fundamental weight, we simplify notation and write $\omega_{\omega_{i}, a}=\omega_{i, a}$. We refer to $\omega_{i, a}$ as a fundamental $\ell$-weight, for all $i \in I$ and $a \in \mathbb{F}^{\times}$. Notice that $\mathscr{P}_{\mathbb{F}}$ is the free abelian group on the set of fundamental $\ell$-weights. One defines $\mathscr{P}_{\mathbb{K}}$ in the obvious way. Let also $\mathscr{P}_{\mathbb{A}}^{\times}$be the submonoid of $\mathscr{P}_{\mathbb{K}}^{+}$generated by $\omega_{i, a}, i \in I, a \in \mathbb{A}^{\times}$.

Let wt $: \mathscr{P}_{\mathbb{F}} \rightarrow P$ be the unique group homomorphism such that $\operatorname{wt}\left(\omega_{i, a}\right)=\omega_{i}$ for all $i \in I, a \in \mathbb{F}^{\times}$. Let also $\omega \mapsto \omega^{-}$be the unique group automorphism of $\mathscr{P}_{\mathbb{F}}$ mapping $\omega_{i, a}$ to $\omega_{i, a^{-1}}$ for all $i \in I, a \in \mathbb{F}^{\times}$. For notational convenience we set $\omega^{+}=\omega$.

The abelian group $\mathscr{P}_{\mathbb{F}}$ can be identified with a subgroup of the monoid of $|I|$-tuples of formal power series with coefficients in $\mathbb{F}$ by identifying the rational function $(1-a u)^{-1}$ with the corresponding geometric formal power series $\sum_{n \geq 0}(a u)^{n}$. This allows us to define an inclusion $\mathscr{P}_{\mathbb{F}} \hookrightarrow U_{\mathbb{F}}(\tilde{\mathfrak{h}})^{*}$. Indeed, if $\omega \in \mathscr{P}_{\mathbb{F}}$ is such that $\boldsymbol{\omega}_{i}^{ \pm}(u)=\sum_{r \geq 0} \omega_{i, \pm r} u^{r} \in \mathscr{P}_{\mathbb{F}}$, set

$$
\omega\left(\left(\begin{array}{c}
h_{i} \\
k
\end{array}\right)\right)=\left(\begin{array}{c}
\operatorname{wt}(\omega)\left(h_{i}\right) \\
k
\end{array}\right), \quad \omega\left(\Lambda_{i, r}\right)=\omega_{i, r}, \quad \text { for all } i \in I, r, k \in \mathbb{Z}, k \geq 0,
$$

and $\omega(x y)=\omega(x) \omega(y)$, for all $x, y \in U_{\mathbb{F}}(\tilde{\mathfrak{h}})$.

1.5. Demazure and local Weyl modules. Given $\omega \in \mathscr{P}_{\mathbb{F}}^{+}$, the local Weyl module $W_{\mathbb{F}}(\omega)$ is the quotient of $U_{\mathbb{F}}(\tilde{\mathfrak{g}})$ by the left ideal generated by

$$
U_{\mathbb{F}}\left(\tilde{\mathfrak{n}}^{+}\right)^{0}, \quad h-\omega(h), \quad\left(x_{\alpha}^{-}\right)^{(k)} \text { for all } h \in U_{\mathbb{F}}(\tilde{\mathfrak{h}}), \alpha \in R^{+}, k>\operatorname{wt}(\omega)\left(h_{\alpha}\right) .
$$

It is known that the local Weyl modules are finite-dimensional (see Theorem 3.2.1(c)).

For $\lambda \in P^{+}$, the graded local Weyl module $W_{\mathbb{F}}^{c}(\lambda)$ is the quotient of $U_{\mathbb{F}}(\mathfrak{g}[t])$ by the left ideal $I_{\mathbb{F}}^{c}(\lambda)$ generated by

$$
U_{\mathbb{F}}\left(\mathfrak{n}^{+}[t]\right)^{0}, \quad U_{\mathbb{F}}\left(\mathfrak{h}[t]_{+}\right)^{0}, \quad h-\lambda(h), \quad\left(x_{\alpha}^{-}\right)^{(k)}
$$

$$
\text { for all } h \in U_{\mathbb{F}}(\mathfrak{h}), \alpha \in R^{+}, k>\lambda\left(h_{\alpha}\right) \text {. }
$$

Also, given $\ell \geq 0$, let $D_{\mathbb{F}}(\ell, \lambda)$ denote the quotient of $U_{\mathbb{F}}(\mathfrak{g}[t])$ by the left ideal $I_{\mathbb{F}}(\ell, \lambda)$ generated by $I_{\mathbb{F}}^{c}(\lambda)$ together with

$$
\left(x_{\alpha, s}^{-}\right)^{(k)} \text { for all } \alpha \in R^{+}, s, k \in \mathbb{Z}_{\geq 0}, k>\max \left\{0, \lambda\left(h_{\alpha}\right)-s \ell r_{\alpha}^{\vee}\right\} .
$$

In particular, $D_{\mathbb{F}}(\ell, \lambda)$ is a quotient of $W_{\mathbb{F}}^{c}(\lambda)$. 
The algebra $U_{\mathbb{F}}(\mathfrak{g}[t])$ inherits a $\mathbb{Z}$-grading from the grading on the polynomial algebra $\mathbb{C}[t]$. The ideals $I_{\mathbb{F}}^{c}(\lambda)$ and $I_{\mathbb{F}}(\ell, \lambda)$ are clearly graded and, hence, the modules $W_{\mathbb{F}}^{c}(\lambda)$ and $D_{\mathbb{E}}(\ell, \lambda)$ are graded. If $V$ is a graded module, let $V[r]$ be its $r$-th graded piece. Given $m \in \mathbb{Z}$, let $\tau_{m}(V)$ be the $U_{\mathbb{F}}(\mathfrak{g}[t])$-module such that $\tau_{s}(V)[r]=V[r-m]$ for all $r \in \mathbb{Z}$. Set

$$
D_{\mathbb{E}}(\ell, \lambda, m)=\tau_{m}\left(D_{\mathbb{F}}(\ell, \lambda)\right) .
$$

Remark 1.5.1. Local Weyl modules were simply called Weyl modules in [Chari and Pressley 2001]. Certain infinite-dimensional modules, which were called maximal integrable modules in the same work, are now called global Weyl modules. The modern names, local and global Weyl modules were coined in [Feigin and Loktev 2004], where the authors introduced these modules in the context of generalized current algebras. We will not consider the global Weyl modules in this paper. We refer the reader to [Chari et al. 2010; Fourier et al. 2012; Fourier et al. 2014] and references therein for recent developments in the theory of global and local Weyl modules for (equivariant) map algebras. See also [Chamberlin 2013] for the initial steps in the study of the hyperalgebras of (equivariant) map algebras.

We are ready to state the main theorem of the paper.

Theorem 1.5.2. Let $\lambda \in P^{+}$.

(a) If $\mathfrak{g}$ is simply laced, then $D_{\mathbb{F}}(1, \lambda)$ and $W_{\mathbb{F}}^{c}(\lambda)$ are isomorphic $U_{\mathbb{F}}(\mathfrak{g}[t])$-modules.

(b) There exist $k \geq 1, m_{j} \in \mathbb{Z}_{\geq 0}$, and $\lambda_{j} \in P^{+}, j=1, \ldots, k$, (independent of $\mathbb{F}$ ) such that the $U_{\mathbb{F}}(\mathfrak{g}[t])$-module $W_{\mathbb{F}}^{c}(\lambda)$ admits a filtration $(0)=W_{0} \subset W_{1} \subset$ $\cdots \subset W_{k-1} \subset W_{k}=W_{\mathbb{F}}^{c}(\lambda)$, with

$$
W_{j} / W_{j-1} \cong D_{\mathbb{F}}\left(1, \lambda_{j}, m_{j}\right) .
$$

(c) For any $a \in \mathbb{F}^{\times}$, there exists an automorphism $\varphi_{a}$ of $U_{\mathbb{F}}(\mathfrak{g}[t])$ such that the pull-back of $W_{\mathbb{E}}\left(\boldsymbol{\omega}_{\lambda, a}\right)$ by $\varphi_{a}$ is isomorphic to $W_{\mathbb{F}}^{c}(\lambda)$.

(d) If $\omega=\prod_{j=1}^{m} \omega_{\lambda_{j}, a_{j}}$ for some $m \geq 0, \lambda_{j} \in P^{+}, a_{j} \in \mathbb{F}^{\times}, j=1, \ldots$, m, with $a_{i} \neq a_{j}$ for $i \neq j$, then

$$
W_{\mathbb{F}}(\boldsymbol{\omega}) \cong \bigotimes_{j=1}^{m} W_{\mathbb{F}}\left(\boldsymbol{\omega}_{\lambda_{j}, a_{j}}\right) .
$$

Assume the characteristic of $\mathbb{E}$ is zero. Then part (a) of this theorem was proved in [Chari and Pressley 2001] for $\mathfrak{g}=\mathfrak{s l}_{2}$, in [Chari and Loktev 2006] for type $A$, and in [Fourier and Littelmann 2007] for types ADE. Part (b) was proved in [Naoi 2012]. Part (c) for simply-laced $\mathfrak{g}$ was proved in [Fourier and Littelmann 2007] using part (a) (see Lemmas 1 and 3 and Equation (15) of that reference). The same proof works in the nonsimply laced case once part (b) is established. The last part 
was proved in [Chari and Pressley 2001]. We will make use of Theorem 1.5.2 in the characteristic zero setting for extending it to the positive characteristic context. Both [Chari and Loktev 2006] and [Fourier and Littelmann 2007] use the $\mathfrak{s l}_{2}$-case of part (a) in the proofs. A characteristic-free proof of Theorem 1.5.2(a) for $\mathfrak{s l}_{2}$ was given in [Jakelić and Moura 2014].

We will see in Section 3.5 that the class of modules $D_{\mathbb{F}}(\ell, \lambda)$ form a subclass of the class of Demazure modules. In particular, it follows from [Mathieu 1988, Lemme 8] that $\operatorname{dim}\left(D_{\mathbb{F}}(\ell, \lambda)\right)$ depends only on $\ell$ and $\lambda$, but not on $\mathbb{F}$ (see also the remark on page 56 of [Mathieu 1989] and references therein). Together with Theorem 1.5.2(b), this implies the following corollary.

Corollary 1.5.3. For all $\lambda \in P^{+}$, we have $\operatorname{dim} W_{\mathbb{E}}^{c}(\lambda)=\operatorname{dim} W_{\mathbb{C}}^{c}(\lambda)$.

As an application of this corollary, we will prove a conjecture of Jakelić and Moura, which we recall after quoting a theorem of theirs.

Theorem 1.5.4 [Jakelić and Moura 2007]. Suppose $\omega \in \mathscr{P}_{\mathbb{A}}^{\times}$and let $\lambda=\operatorname{wt}(\omega), v$ be the image of 1 in $W_{\mathbb{K}}(\omega)$, and $L_{\mathbb{A}}(\omega)=U_{\mathbb{A}}(\tilde{\mathfrak{g}}) v$. Then $L_{\mathbb{A}}(\omega)$ is a free $\mathbb{A}$-module such that $\mathbb{R} \otimes_{\mathbb{A}} L_{\mathbb{A}}(\omega) \cong W_{\mathbb{K}}(\omega)$.

Let $\varpi$ be the image of $\omega$ in $\mathscr{P}_{\mathbb{F}}$. It easily follows that $\mathbb{F} \otimes_{\mathbb{A}} L_{\mathbb{A}}(\omega)$ is a quotient of $W_{\mathbb{F}}(\varpi)$ and, hence,

$$
\operatorname{dim} W_{\mathbb{K}}(\boldsymbol{\omega}) \leq \operatorname{dim} W_{\mathbb{F}}(\varpi) .
$$

It was conjectured in [Jakelić and Moura 2007] that

$$
\mathbb{F} \otimes_{\mathbb{A}} L_{\mathbb{A}}(\omega) \cong W_{\mathbb{F}}(\varpi) .
$$

We will prove (1.5.4) in Section 3.4. In particular, it follows that

$$
\operatorname{dim} W_{\mathbb{F}}(\varpi)=\operatorname{dim} W_{\mathbb{C}}^{c}(\lambda) .
$$

Remark 1.5.5. Theorem 1.5.2(d) was also conjectured in [Jakelić and Moura 2007] and it is false if $\mathbb{F}$ were not algebraically closed (see [Jakelić and Moura 2010] in that case). Observe that for all $\varpi \in \mathscr{P}_{\mathbb{F}}^{+}$there exists $\omega \in \mathscr{P}_{\mathbb{A}}^{\times}$such that $\varpi$ is the image of $\omega$ in $\mathscr{P}_{\mathbb{F}}$. This is the main reason for considering $\mathbb{A}$-forms instead of $\mathbb{Z}$-forms. The block decomposition of the categories of finite-dimensional representations of hyper loop algebras was established in [Jakelić and Moura 2007; 2010] assuming (1.5.4) and Theorem 1.5.2(d). The proof of one part of [Bianchi and Moura 2014, Theorem 4.1] also relies on these two results. Therefore, by proving (1.5.4) and Theorem 1.5.2(d), we confirm these results of [Bianchi and Moura 2014; Jakelić and Moura 2007; 2010]. A version of Theorem 1.5.2 for twisted affine Kac-Moody algebras was obtained in [Fourier and Kus 2013] in the characteristic-zero setting. We will consider the characteristic-free twisted version of Theorem 1.5.2 elsewhere. 


\section{Further notation and technical lemmas}

2.1. Some commutation relations. We begin recalling the following well-known relation in $U_{\mathbb{Z}}(\mathfrak{g})$

$$
\left(x_{\alpha}^{+}\right)^{(l)}\left(x_{\alpha}^{-}\right)^{(k)}=\sum_{m=0}^{\min \{k, l\}}\left(x_{\alpha}^{-}\right)^{(k-m)}\left(\begin{array}{c}
h_{\alpha}-k-l+2 m \\
m
\end{array}\right)\left(x_{\alpha}^{+}\right)^{(l-m)}
$$

for all $\alpha \in R^{+}, l, k \in \mathbb{Z}_{\geq 0}$. Since for all $\alpha \in R^{+}, s \in \mathbb{Z}$, the span of $x_{\alpha, \pm s}^{ \pm}, h_{\alpha}$ is a subalgebra isomorphic to $\mathfrak{s l}_{2}$, we get the following relation in $U_{\mathbb{Z}}(\tilde{\mathfrak{g}})$

$$
\left(x_{\alpha, s}^{+}\right)^{(l)}\left(x_{\alpha,-s}^{-}\right)^{(k)}=\sum_{m=0}^{\min \{k, l\}}\left(x_{\alpha,-s}^{-}\right)^{(k-m)}\left(\begin{array}{c}
h_{\alpha}-k-l+2 m \\
m
\end{array}\right)\left(x_{\alpha, s}^{+}\right)^{(l-m)} .
$$

Next, we consider the case when the grades of the elements in the left-hand side is not symmetric.

Given $m>0$, consider the Lie algebra endomorphism $\tau_{m}$ of $\tilde{\mathfrak{g}}$ induced by the ring endomorphism of $\mathbb{C}\left[t, t^{-1}\right], t \mapsto t^{m}$. Notice that the restriction of $\tau_{m}$ to $\mathfrak{g}[t]$ gives rise to an endomorphism of $\mathfrak{g}[t]$. Moreover, denoting by $\tau_{m}$ its extension to an algebra endomorphism of $U(\tilde{\mathfrak{g}})$, notice that $U_{\mathbb{Z}}(\mathfrak{a})$ is invariant under $\tau_{m}$ for $\mathfrak{a}=\mathfrak{g}, \mathfrak{n}^{ \pm}, \mathfrak{h}, \tilde{\mathfrak{n}}^{ \pm}, \tilde{\mathfrak{h}}, \mathfrak{n}^{ \pm}[t], \mathfrak{h}[t], \mathfrak{h}[t]_{+}$. In fact $\tau_{m}\left(\left(x_{\alpha, r}^{ \pm}\right)^{(k)}\right)=\left(x_{\alpha, m r}^{ \pm}\right)^{(k)}$ and $\tau_{m}\left(\Lambda_{\alpha, r}\right)$ satisfies $\sum_{i \geq 0} \tau_{m}\left(\Lambda_{\alpha, r}\right) u^{r}=\exp \left(-\sum_{s \geq 1} \frac{h_{\alpha, m s}}{s} u^{s}\right)$ for all $r, m \in \mathbb{Z}$ and $\alpha \in R^{+}$. Consider the power series

$$
X_{\alpha, m, s}^{-}(u)=\sum_{r=1}^{\infty} x_{\alpha, m(r-1)+s}^{-} u^{r} \quad \text { and } \quad \Lambda_{\alpha, m}^{ \pm}(u)=\tau_{m}\left(\Lambda_{\alpha}^{ \pm}(u)\right) .
$$

Lemma 2.1.1. Let $\alpha \in R^{+}, k, l \geq 0, m>0, s \in \mathbb{Z}$. Then

$$
\left(x_{\alpha, m-s}^{+}\right)^{(l)}\left(x_{\alpha, s}^{-}\right)^{(k)}=(-1)^{l}\left(\left(X_{\alpha, m, s}^{-}(u)\right)^{(k-l)} \Lambda_{\alpha, m}^{+}(u)\right)_{k} \quad \bmod U_{\mathbb{Z}}(\tilde{\mathfrak{g}}) U_{\mathbb{Z}}\left(\tilde{\mathfrak{n}}^{+}\right)^{0},
$$

where the subindex $k$ denotes the coefficient of $u^{k}$ of the above power series. Moreover, if $0 \leq s \leq m$, the same holds modulo $U_{\mathbb{Z}}(\mathfrak{g}[t]) U_{\mathbb{Z}}\left(\mathfrak{n}^{+}[t]\right)_{\mathbb{Z}}^{0}$.

Proof. The case $m=1, s=0$ was proved in [Garland 1978, Lemma 7.5] (see [Jakelić and Moura 2007, Equation (1-11)]). Consider the Lie algebra endomorphism $\sigma_{s}: \tilde{\mathfrak{s l}}_{\alpha} \rightarrow \tilde{\mathfrak{s l}}_{\alpha}$ given by $x_{\alpha, r}^{ \pm} \mapsto x_{\alpha, r \mp s}^{ \pm}$. The first statement of the lemma is obtained from the case $m=1, s=0$ by applying $\left(\sigma_{s} \circ \tau_{m}\right)$. The second statement is then clear.

Sometimes it will be convenient to work with a smaller set of generators for the hyperalgebras.

Proposition 2.1.2 [Mitzman 1985, Corollary 4.4.12]. The ring $U_{\mathbb{Z}}\left(\hat{\mathfrak{g}}^{\prime}\right)$ is generated by $\left(x_{i}^{ \pm}\right)^{(k)}, i \in \hat{I}, k \geq 0$ and $U_{\mathbb{Z}}(\mathfrak{g})$ is generated by $\left(x_{i}^{ \pm}\right)^{(k)}, i \in I, k \geq 0$. 
2.2. On certain automorphisms of hyper current algebras. Let $\mathfrak{a}, \mathfrak{b}$ be such that $U_{\mathbb{Z}}(\mathfrak{a})$ have been defined. Then, given a homomorphism of $\mathbb{A}$-algebras $f: U_{\mathbb{A}}(\mathfrak{a}) \rightarrow$ $U_{\mathbb{A}}(\mathfrak{b})$, we have an induced homomorphism $U_{\mathbb{F}}(\mathfrak{a}) \rightarrow U_{\mathbb{F}}(\mathfrak{b})$. We will now use this procedure to define certain homomorphism between hyperalgebras. As a rule, we shall use the same symbol to denote the induced homomorphism in the hyperalgebra level.

Recall that there exists a unique involutive Lie algebra automorphism $\psi$ of $\mathfrak{g}$ such that $x_{i}^{ \pm} \mapsto x_{i}^{\mp}$ and $h_{i} \mapsto-h_{i}$ for all $i \in I$. It admits a unique extension to an automorphism of $\mathfrak{g}[t]$ such that $\psi(x \otimes f(t))=\psi(x) \otimes f(t)$ for all $x \in \mathfrak{g}, f \in \mathbb{C}[t]$. Keep denoting by $\psi$ its extension to an automorphism of $U(\mathfrak{g}[t])$. In particular, it easily follows that

$$
\psi\left(\left(x_{\alpha, r}^{ \pm}\right)^{(k)}\right)=\left(x_{\alpha, r}^{\mp}\right)^{(k)} \text { for all } \alpha \in R^{+}, r, k \geq 0 .
$$

Since $U_{\mathbb{Z}}(\mathfrak{g}[t])$ is generated by the elements $\left(x_{\alpha, r}^{ \pm}\right)^{(k)}$, it follows that the restriction of $\psi$ to $U_{\mathbb{Z}}(\mathfrak{a})$ induces an automorphism of $U_{\mathbb{Z}}(\mathfrak{a})$, for $\mathfrak{a}=\mathfrak{g}, \mathfrak{h}, \mathfrak{g}[t], \mathfrak{h}[t], \mathfrak{h}[t]_{+}$. We have an inclusion $P \hookrightarrow \operatorname{Hom}_{\mathbb{Z}}\left(U_{\mathbb{Z}}(\mathfrak{h}), \mathbb{Z}\right)$ determined by

$$
\mu\left(\left(\begin{array}{c}
h_{i} \\
k
\end{array}\right)\right)=\left(\begin{array}{c}
\mu\left(h_{i}\right) \\
k
\end{array}\right) \text { and } \mu(x y)=\mu(x) \mu(y)
$$

for all $i \in I, k \geq 0, x, y \in U_{\mathbb{Z}}(\mathfrak{h})$.

Therefore,

$$
\mu\left(\psi\left(\left(\begin{array}{c}
h_{i} \\
k
\end{array}\right)\right)\right)=\left(\begin{array}{c}
-\mu\left(h_{i}\right) \\
k
\end{array}\right) \text { for all } i \in I, k>0, \mu \in P .
$$

Suppose now that $\gamma$ is a Dynkin diagram automorphism of $\mathfrak{g}$ and keep denoting by $\gamma$ the $\mathfrak{g}$-automorphism determined by $x_{i}^{ \pm} \mapsto x_{\gamma(i)}^{ \pm}, h_{i} \mapsto h_{\gamma(i)}, i \in I$. It admits a unique extension to an automorphism of $\mathfrak{g}[t]$ such that $\gamma(x \otimes f(t))=\gamma(x) \otimes f(t)$ for all $x \in \mathfrak{g}, f \in \mathbb{C}[t]$. Keep denoting by $\gamma$ its extension to an automorphism of $U(\mathfrak{g}[t])$. Let $\gamma$ also denote the associated automorphism of $P$ determined by $\gamma\left(\omega_{i}\right)=\omega_{\gamma(i)}, i \in I$. In particular, $\gamma\left(\alpha_{i}\right)=\alpha_{\gamma(i)}, i \in I$. It then follows that for each $\alpha \in R^{+}, k>0$, there exist $\varepsilon_{\alpha, k}^{ \pm} \in\{-1,1\}$ (depending on how the Chevalley basis was chosen) such that

$$
\gamma\left(\left(x_{\alpha, r}^{ \pm}\right)^{(k)}\right)=\varepsilon_{\alpha, k}^{ \pm}\left(x_{\gamma(\alpha), r}^{ \pm}\right)^{(k)} \text { for all } r \geq 0 .
$$

This implies that the restriction of $\gamma$ to $U_{\mathbb{Z}}(\mathfrak{a})$ induces an automorphism of $U_{\mathbb{Z}}(\mathfrak{a})$, for any $\mathfrak{a}$ in the set $\left\{\mathfrak{g}, \mathfrak{n}^{ \pm}, \mathfrak{h}, \mathfrak{g}[t], \mathfrak{n}^{ \pm}[t], \mathfrak{h}[t], \mathfrak{h}[t]_{+}\right\}$. It is also easy to see that

$$
\mu\left(\gamma\left(\left(\begin{array}{c}
h_{i} \\
k
\end{array}\right)\right)\right)=\left(\begin{array}{c}
\left(\gamma^{-1}(\mu)\right)\left(h_{i}\right) \\
k
\end{array}\right) \text { for all } i \in I, k>0, \mu \in P .
$$

We conclude this subsection by constructing the automorphism mentioned in 
Theorem 1.5.2(c). Thus, let $a \in \mathbb{F}$ and $\tilde{a} \in \mathbb{A}$ be such that the image of $\tilde{a}$ in $\mathbb{F}$ is $a$, and $\varphi_{\tilde{a}}$ the Lie algebra automorphism of $\mathfrak{g}[t]_{\mathbb{K}}$ given by $x \otimes t \mapsto x \otimes(t-\tilde{a})$. Keep denoting by $\varphi_{\tilde{a}}$ the induced automorphism of $U_{\mathbb{K}}(\mathfrak{g}[t])$ and observe that $\varphi_{\tilde{a}}$ is the identity on $U_{\mathbb{}}(\mathfrak{g})$. One easily checks that

$$
\varphi_{\tilde{a}}\left(\left(x_{\alpha, r}^{ \pm}\right)^{(k)}\right)=\sum_{k_{0}+\cdots+k_{r}=k} \prod_{s=0}^{r}\left(\begin{array}{l}
r \\
s
\end{array}\right)^{k_{s}}(-\tilde{a})^{k_{s}(r-s)}\left(x_{\alpha, s}^{ \pm}\right)^{\left(k_{s}\right)} \in U_{\mathbb{A}}(\mathfrak{g}[t]) .
$$

Hence, $\varphi_{\tilde{a}}$ induces an automorphism of $U_{\mathbb{A}}(\mathfrak{g}[t])$. Notice that in the hyperalgebra level we have

$$
\left(x_{\alpha, r}^{ \pm}\right)^{(k)} \mapsto \sum_{k_{0}+\cdots+k_{r}=k} \prod_{s=0}^{r}\left(\begin{array}{l}
r \\
s
\end{array}\right)^{k_{s}}(-a)^{k_{s}(r-s)}\left(x_{\alpha, s}^{ \pm}\right)^{\left(k_{s}\right)} .
$$

This justifies a change of notation from $\varphi_{\tilde{a}}$ to $\varphi_{a}$.

2.3. Subalgebras of rank 1 and 2. For any $\alpha \in R^{+}$, consider the Lie subalgebra of $\mathfrak{g}$ generated by $x_{\alpha}^{ \pm}$which is isomorphic to $\mathfrak{s l}_{2}$. Denote this subalgebra by $\mathfrak{s l}_{\alpha}$. Consider also $\mathfrak{n}_{\alpha}^{ \pm}=\mathbb{C} x_{\alpha}^{ \pm}, \mathfrak{h}_{\alpha}=\mathbb{C} h_{\alpha}$ and $\mathfrak{b}_{\alpha}^{ \pm}=\mathbb{C} h_{\alpha} \oplus \mathbb{C} x_{\alpha}^{ \pm}$. Notice that $U_{\mathbb{Z}}(\mathfrak{g}) \cap U(\mathfrak{s l})$ coincides with the $\mathbb{Z}$-subalgebra $U_{\mathbb{Z}}\left(\mathfrak{s l}_{\alpha}\right)$ of $U(\mathfrak{g})$ generated by $\left(x_{\alpha}^{ \pm}\right)^{(k)}, k \geq 0$ (see details in [Macedo 2013]). This implies that $U_{\mathbb{Z}}(\mathfrak{g}) \cap U\left(\mathfrak{s l}_{\alpha}\right)$ is naturally isomorphic to $U_{\mathbb{Z}}\left(\mathfrak{s l}_{2}\right)$ and, hence, the corresponding subalgebra $U_{\mathbb{F}}\left(\mathfrak{s l}_{\alpha}\right)$ of $U_{\mathbb{F}}(\tilde{\mathfrak{g}})$ is naturally isomorphic to $U_{\mathbb{F}}\left(\mathfrak{s l}_{2}\right)$. Similarly, for any $\alpha \in R^{+}, r \in \mathbb{Z}$, the Lie subalgebra $\mathfrak{s l}_{\alpha, r}$ of $\tilde{\mathfrak{g}}$ generated by $x_{\alpha, \pm r}^{ \pm}$is isomorphic to $\mathfrak{s l}_{2}$ and $U_{\mathbb{Z}}(\tilde{\mathfrak{g}}) \cap U\left(\mathfrak{s l}_{\alpha, r}\right)$ coincides with the $\mathbb{Z}$-subalgebra of $U(\tilde{\mathfrak{g}})$ generated by $\left(x_{\alpha, \pm r}^{ \pm}\right)^{(k)}, k \geq 0$. We shall denote the corresponding subalgebra of $U_{\mathbb{F}}(\tilde{\mathfrak{g}})$ by $U_{\mathbb{F}}\left(\mathfrak{s l}_{\alpha, r}\right)$. We also consider the subalgebra $\tilde{\mathfrak{s l}}_{\alpha}$ of $\tilde{\mathfrak{g}}$ generated by $x_{\alpha, r}^{ \pm}, r \in \mathbb{Z}$ and the subalgebra $\mathfrak{s l}_{\alpha}[t]$ of $\mathfrak{g}[t]$ generated by $x_{\alpha, r}^{ \pm}, r \geq 0$. The corresponding subalgebras $U_{\mathbb{F}}\left(\tilde{\mathfrak{s l}}_{\alpha}\right)$ and $U_{\mathbb{F}}\left(\mathfrak{s l}_{\alpha}[t]\right)$ of $U_{\mathbb{F}}(\tilde{\mathfrak{g}})$ are naturally isomorphic to $U_{\mathbb{F}}\left(\tilde{\mathfrak{s}}_{2}\right)$ and $U_{\mathbb{F}}\left(\mathfrak{s l}_{2}[t]\right)$.

We will also need to work with root subsystems of rank 2. Suppose $\alpha, \beta \in R^{+}$ form a simple system of a root subsystem $R^{\prime}$ of rank 2 and let $\mathfrak{t}$ denote a simple Lie algebra of type $R^{\prime}$. Denote by $\mathfrak{g}_{\alpha, \beta}$ the subalgebra of $\mathfrak{g}$ generated by $x_{\alpha}^{ \pm}$and $x_{\beta}^{ \pm}$, which is isomorphic to $\mathfrak{t}$. Notice that, for $r, s \in \mathbb{Z}$, the subalgebra $\mathfrak{g}_{\alpha, \beta}^{r, s}$ of $\tilde{\mathfrak{g}}$ generated by $x_{\alpha, \pm r}^{ \pm}$and $x_{\beta, \pm s}^{ \pm}$is also isomorphic to $t$. Let $U_{\mathbb{Z}}^{\prime}\left(\mathfrak{g}_{\alpha, \beta}\right)$ be the subalgebra of $U_{\mathbb{Z}}(\mathfrak{g})$ generated by $\left(x_{\alpha}^{ \pm}\right)^{(k)},\left(x_{\beta}^{ \pm}\right)^{(k)}, k \geq 0$, and $U_{\mathbb{Z}}^{\prime}\left(\mathfrak{g}_{\alpha, \beta}^{r, s}\right)$ the subalgebra of $U_{\mathbb{Z}}(\tilde{\mathfrak{g}})$ generated by $\left(x_{\alpha, \pm r}^{ \pm}\right)^{(k)},\left(x_{\beta, \pm s}^{ \pm}\right)^{(k)}, k \geq 0$. Proposition 2.1.2 implies that $U_{\mathbb{Z}}^{\prime}\left(\mathfrak{g}_{\alpha, \beta}\right)$ and $U_{\mathbb{Z}}^{\prime}\left(\mathfrak{g}_{\alpha, \beta}^{r, s}\right)$ are naturally isomorphic to $U_{\mathbb{Z}}(\mathfrak{t})$. Recall that if $\mathfrak{a}$ is a subalgebra of $U(\tilde{\mathfrak{g}})$, then $U_{\mathbb{Z}}(\mathfrak{a})=U(\mathfrak{a}) \cap U_{\mathbb{Z}}(\tilde{\mathfrak{g}})$. As in the rank-1 case, we have

$$
U_{\mathbb{Z}}^{\prime}\left(\mathfrak{g}_{\alpha, \beta}\right)=U_{\mathbb{Z}}\left(\mathfrak{g}_{\alpha, \beta}\right) \quad \text { and } \quad U_{\mathbb{Z}}^{\prime}\left(\mathfrak{g}_{\alpha, \beta}^{r, s}\right)=U_{\mathbb{Z}}\left(\mathfrak{g}_{\alpha, \beta}^{r, s}\right) .
$$


The details can be found in [Macedo 2013]. It follows from (2.3.1) that $U_{\mathbb{F}}\left(\mathfrak{g}_{\alpha, \beta}\right)=$ $\mathbb{F} \otimes_{\mathbb{Z}} U_{\mathbb{Z}}\left(\mathfrak{g}_{\alpha, \beta}\right) \subseteq U_{\mathbb{F}}(\mathfrak{g})$ and $U_{\mathbb{F}}\left(\mathfrak{g}_{\alpha, \beta}^{r, s}\right)=\mathbb{F} \otimes_{\mathbb{Z}} U_{\mathbb{Z}}\left(\mathfrak{g}_{\alpha, \beta}^{r, s}\right) \subseteq U_{\mathbb{F}}(\tilde{\mathfrak{g}})$ are isomorphic to $U_{\mathbb{E}}(\mathfrak{t})$.

2.4. The algebra $\mathfrak{g}_{\mathrm{sh}}$. Another important subalgebra used in the proof of our main result is the subalgebra $\mathfrak{g}_{\mathrm{sh}}$ generated by the root vectors associated to short simple roots.

Let $\Delta_{\mathrm{sh}}=\{\alpha \in \Delta:(\alpha, \alpha)<2\}$ denote the set of simple short roots. In particular, if $\mathfrak{g}$ is simply laced, $\Delta_{\mathrm{sh}}=\varnothing$. Let $R_{\mathrm{sh}}^{+}=\mathbb{Z} \Delta_{\mathrm{sh}} \cap R^{+}$and $R_{\mathrm{sh}}=\mathbb{Z} \Delta_{\mathrm{sh}} \cap R$ (and notice that if $\mathfrak{g}$ is not simply laced, $\left.R_{\mathrm{sh}} \neq\{\alpha \in R:(\alpha, \alpha)<2\}\right)$. Set $I_{\mathrm{sh}}=\left\{i \in I: \alpha_{i} \in \Delta_{\mathrm{sh}}\right\}$ and define $P_{\mathrm{sh}}=\bigoplus_{i \in I_{\mathrm{sh}}} \mathbb{Z} \omega_{i}$ and $P_{\mathrm{sh}}^{+}=P_{\mathrm{sh}} \cap P^{+}$. Consider also the subalgebras $\mathfrak{h}_{\mathrm{sh}}=\bigoplus_{i \in I_{\mathrm{sh}}} \mathbb{C} h_{i}, \mathfrak{b}_{\mathrm{sh}}^{ \pm}=\mathfrak{h}_{\mathrm{sh}} \oplus \mathfrak{n}_{\mathrm{sh}}^{ \pm}$, where $\mathfrak{n}_{\mathrm{sh}}^{ \pm}=\bigoplus_{ \pm \alpha \in R_{\mathrm{sh}}^{+}} \mathfrak{g}_{\alpha}$, and $\mathfrak{g}_{\mathrm{sh}}=\mathfrak{n}_{\mathrm{sh}}^{-} \oplus \mathfrak{h}_{\mathrm{sh}} \oplus \mathfrak{n}_{\mathrm{sh}}^{+}$. Then if $\Delta_{\mathrm{sh}} \neq \varnothing, \mathfrak{g}_{\mathrm{sh}}$ is a simply laced Lie subalgebra of $\mathfrak{g}$ with Cartan subalgebra $\mathfrak{h}_{\mathrm{sh}}$ and $\Delta_{\text {sh }}$ can be identified with the choice of simple roots associated to the given triangular decomposition. The subsets $Q_{\mathrm{sh}}, Q_{\mathrm{sh}}^{+}$, and the Weyl group $\mathcal{W}_{\mathrm{sh}}$ are defined in the obvious way. The restriction of $($,$) to \mathfrak{g}_{\mathrm{sh}}$ is an invariant symmetric and nondegenerate bilinear form on $\mathfrak{g}_{\mathrm{sh}}$, but the normalization is not the same as the one we fixed for $\mathfrak{g}$. Indeed, $(\alpha, \alpha)=2 / r^{\vee}$ for all $\alpha \in R_{\mathrm{sh}}$. The set $\left\{x_{\alpha}^{ \pm}, h_{i}: \alpha \in R_{\mathrm{sh}}^{+}\right.$, $\left.i \in I_{\text {sh }}\right\}$ is a Chevalley basis for $\mathfrak{g}_{\mathrm{sh}}$.

Observe that $U_{\mathbb{Z}}(\mathfrak{g}) \cap U\left(\mathfrak{g}_{\mathrm{sh}}\right)$ coincides with the $\mathbb{Z}$-subalgebra of $U(\mathfrak{g})$ generated by $\left(x_{\alpha}^{ \pm}\right)^{(k)}, \alpha \in \Delta_{\mathrm{sh}}$; and, hence, Proposition 2.1.2 implies that $U_{\mathbb{F}}\left(\mathfrak{g}_{\mathrm{sh}}\right)$ can be naturally identified with a subalgebra of $U_{\mathbb{F}}(\mathfrak{g})$. Similar observation apply to $U_{\mathbb{Z}}(\mathfrak{a})$ for $\mathfrak{a}=\mathfrak{n}_{\mathrm{sh}}^{ \pm}, \mathfrak{h}_{\mathrm{sh}}$.

Consider the linear map $\mathfrak{h}^{*} \rightarrow \mathfrak{h}_{\mathrm{sh}}^{*}, \lambda \mapsto \bar{\lambda}$, given by restriction and let $i_{\mathrm{sh}}: \mathfrak{h}_{\mathrm{sh}}^{*} \rightarrow \mathfrak{h}^{*}$ be the linear map such that $i_{\mathrm{sh}}(\bar{\alpha})=\alpha$ for all $\alpha \in \Delta_{\mathrm{sh}}$. In particular, $\overline{i_{\mathrm{sh}}(\mu)}=\mu$ for all $\mu \in \mathfrak{h}_{\mathrm{sh}}^{*}$. Given $\lambda \in P$, consider the function $\eta_{\lambda}: P_{\mathrm{sh}} \rightarrow P$ given by

$$
\eta_{\lambda}(\mu)=i_{\mathrm{sh}}(\mu)+\lambda-i_{\mathrm{sh}}(\bar{\lambda}) .
$$

Lemma 2.4.1. If $\lambda \in P^{+}, \mu \in P_{\mathrm{sh}}^{+}$, and $\mu \leq \bar{\lambda}$, then $\eta_{\lambda}(\mu) \in P^{+}$.

Proof. For each $i \in I_{\text {sh }}$, take $m_{i} \in \mathbb{Z}_{\geq 0}$ such that $\mu=\bar{\lambda}-\sum_{i \in I_{\text {sh }}} m_{i} \bar{\alpha}_{i}$. In particular, $\eta_{\lambda}(\mu)=\lambda-\sum_{i \in I_{\text {sh }}} m_{i} \alpha_{i}$. Then for $j \in I_{\text {sh }}$ we have $\eta_{\lambda}(\mu)\left(h_{j}\right)=\mu\left(h_{j}\right) \geq 0$, while for $j \in I \backslash I_{\text {sh }}$ we have $\eta_{\lambda}(\mu)\left(h_{j}\right)=\lambda\left(h_{j}\right)-\sum_{i \in I_{\text {sh }}} m_{i} \alpha_{i}\left(h_{j}\right) \geq \lambda\left(h_{j}\right) \geq 0$.

The affine Kac-Moody algebra associated to $\mathfrak{g}_{\mathrm{sh}}$ is naturally isomorphic to the subalgebra

$$
\hat{\mathfrak{g}}_{\mathrm{sh}}:=\mathfrak{g}_{\mathrm{sh}} \otimes \mathbb{C}\left[t, t^{-1}\right] \oplus \mathbb{C} c \oplus \mathbb{C} d
$$

of $\hat{\mathfrak{g}}$, and under this isomorphism $\hat{\mathfrak{h}}_{\mathrm{sh}}$ is identified with $\mathfrak{h}_{\mathrm{sh}} \oplus \mathbb{C} c \oplus \mathbb{C} d$. The subalgebras $\mathfrak{g}_{\mathrm{sh}}[t]$ and $\hat{\mathfrak{n}}_{\mathrm{sh}}^{ \pm}$, as well as $\hat{P}_{\mathrm{sh}}, \hat{Q}_{\mathrm{sh}}$, etc., are defined in the obvious way. Moreover, $U_{\mathbb{F}}\left(\tilde{\mathfrak{g}}_{\mathrm{sh}}\right)$ and $U_{\mathbb{F}}\left(\mathfrak{g}_{\mathrm{sh}}[t]\right)$ can be naturally identified with a subalgebra of $U_{\mathbb{F}}(\tilde{\mathfrak{g}})$. 


\section{Finite-dimensional modules}

3.1. Modules for hyperalgebras. We now review the finite-dimensional representation theory of $U_{\mathbb{F}}(\mathfrak{g})$. If the characteristic of $\mathbb{F}$ is zero, then $U_{\mathbb{F}}(\mathfrak{g}) \cong U\left(\mathfrak{g}_{\mathbb{F}}\right)$ and the results stated here can be found in [Humphreys 1978]. The literature for the positive characteristic setting is more often found in the context of algebraic groups, in which case $U_{\mathbb{F}}(\mathfrak{g})$ is known as the hyperalgebra or algebra of distributions of an algebraic group of the same Lie type as $\mathfrak{g}$ (see Part II of [Jantzen 2003]). A more detailed review in the present context can be found in [Jakelić and Moura 2007, Section 2].

Let $V$ be a $U_{\mathbb{F}}(\mathfrak{g})$-module. A nonzero vector $v \in V$ is called a weight vector if there exists $\mu \in U_{\mathbb{F}}(\mathfrak{h})^{*}$ such that $h v=\mu(h) v$ for all $h \in U_{\mathbb{F}}(\mathfrak{h})$. The subspace consisting of weight vectors of weight $\mu$ is called a weight space of weight $\mu$ and it will be denoted by $V_{\mu}$. If $V=\bigoplus_{\mu \in U_{\mathbb{F}}(\mathfrak{h})^{*}} V_{\mu}$, then $V$ is said to be a weight module. If $V_{\mu} \neq 0, \mu$ is said to be a weight of $V$ and $\operatorname{wt}(V)=\left\{\mu \in U_{\mathbb{F}}(\mathfrak{h})^{*}: V_{\mu} \neq 0\right\}$ is said to be the set of weights of $V$. Notice that the inclusion (2.2.2) induces an inclusion $P \hookrightarrow U_{\mathbb{E}}(\mathfrak{h})^{*}$. In particular, we can consider the partial order $\leq$ on $U_{\mathbb{F}}(\mathfrak{h})^{*}$ given by $\mu \leq \lambda$ if $\lambda-\mu \in Q^{+}$and we have

$$
\left(x_{\alpha}^{ \pm}\right)^{(k)} V_{\mu} \subseteq V_{\mu \pm k \alpha} \text { for all } \alpha \in R^{+}, k>0, \mu \in U_{\mathbb{F}}(\mathfrak{h})^{*} .
$$

If $V$ is a weight-module with finite-dimensional weight spaces, its character is the function $\operatorname{ch}(V): U_{\mathbb{F}}(\mathfrak{h})^{*} \rightarrow \mathbb{Z}$ given by $\operatorname{ch}(V)(\mu)=\operatorname{dim} V_{\mu}$. As usual, if $V$ is finitedimensional, $\operatorname{ch}(V)$ can be regarded as an element of the group ring $\mathbb{Z}\left[U_{\mathbb{F}}(\mathfrak{h})^{*}\right]$ where we denote the element corresponding to $\mu \in U_{\mathbb{F}}(\mathfrak{h})^{*}$ by $e^{\mu}$. By the inclusion (2.2.2) the group ring $\mathbb{Z}[P]$ can be regarded as a subring of $\mathbb{Z}\left[U_{\mathbb{F}}(\mathfrak{h})^{*}\right]$ and, moreover, the action of $\mathscr{W}$ on $P$ induces an action of $\mathscr{W}$ on $\mathbb{Z}[P]$ by ring automorphisms where $w \cdot e^{\mu}=e^{w \mu}$.

If $v \in V$ is weight vector such that $\left(x_{\alpha}^{+}\right)^{(k)} v=0$ for all $\alpha \in R^{+}, k>0$, then $v$ is said to be a highest-weight vector. If $V$ is generated by a highest-weight vector, then it is said to be a highest-weight module. Similarly, one defines the notions of lowest-weight vectors and modules by replacing $\left(x_{\alpha}^{+}\right)^{(k)}$ by $\left(x_{\alpha}^{-}\right)^{(k)}$.

Theorem 3.1.1. Let $V$ be a $U_{\mathbb{F}}(\mathfrak{g})$-module.

(a) If $V$ is finite-dimensional, then $V$ is a weight-module, $\operatorname{wt}(V) \subseteq P$, and $\operatorname{dim} V_{\mu}=\operatorname{dim} V_{\sigma \mu}$ for all $\sigma \in \mathcal{W}, \mu \in U_{\mathbb{F}}(\mathfrak{h})^{*}$. In particular, $\operatorname{ch}(V) \in \mathbb{Z}[P]^{\mathscr{W}}$.

(b) If $V$ is a highest-weight module of highest weight $\lambda$, then $\operatorname{dim}\left(V_{\lambda}\right)=1$ and $V_{\mu}$ is nonzero only if $\mu \leq \lambda$. Moreover, $V$ has a unique maximal proper submodule and, hence, also a unique irreducible quotient. In particular, $V$ is indecomposable. 
(c) For each $\lambda \in P^{+}$, the $U_{\mathbb{F}}(\mathfrak{g})$-module $W_{\mathbb{F}}(\lambda)$ given by the quotient of $U_{\mathbb{F}}(\mathfrak{g})$ by the left ideal $I_{\mathbb{F}}(\lambda)$ generated by

$$
U_{\mathbb{E}}\left(\mathfrak{n}^{+}\right)^{0}, \quad h-\lambda(h) \quad \text { and } \quad\left(x_{\alpha}^{-}\right)^{(k)}, \text { for all } h \in U_{\mathbb{F}}(\mathfrak{h}), \alpha \in R^{+}, k>\lambda\left(h_{\alpha}\right),
$$

is nonzero and finite-dimensional. Moreover, every finite-dimensional highestweight module of highest weight $\lambda$ is a quotient of $W_{\mathbb{E}}(\lambda)$.

(d) If $V$ is finite-dimensional and irreducible, then there exists a unique $\lambda \in P^{+}$ such that $V$ is isomorphic to the irreducible quotient $V_{\mathbb{F}}(\lambda)$ of $W_{\mathbb{F}}(\lambda)$. If the characteristic of $\mathbb{E}$ is zero, then $W_{\mathbb{F}}(\lambda)$ is irreducible.

(e) For each $\lambda \in P^{+}, \operatorname{ch}\left(W_{\mathbb{F}}(\lambda)\right)$ is given by the Weyl character formula. In particular, $\mu \in \operatorname{wt}\left(W_{\mathbb{F}}(\lambda)\right)$ if and only if $\sigma \mu \leq \lambda$ for all $\sigma \in \mathcal{W}$. Moreover, $W_{\mathbb{E}}(\lambda)$ is a lowest-weight module with lowest weight $w_{0} \lambda$.

Remark 3.1.2. The module $W_{\mathbb{F}}(\lambda)$ defined in Theorem 3.1.1(c) is called the Weyl module (or costandard module) of highest weight $\lambda$. The known proofs of Theorem 3.1.1(e) make use of geometric results such as Kempf's Vanishing Theorem.

We shall need the following lemma in the proof of Lemma 5.2.5 below.

Lemma 3.1.3. Let $V$ be a finite-dimensional $U_{\mathbb{F}}(\mathfrak{g})$-module, $\mu \in P$, and $\alpha \in R^{+}$. If $v \in V_{\mu} \backslash\{0\}$ is such that $\left(x_{\alpha}^{-}\right)^{(k)} v=0$ for all $k>0$, then $\mu\left(h_{\alpha}\right) \in \mathbb{Z}_{\leq 0}$ and $\left(x_{\alpha}^{+}\right)^{\left(-\mu\left(h_{\alpha}\right)\right)} v \neq 0$.

Remark 3.1.4. In characteristic zero, it is well known that the following stronger statement holds: if $v \in V_{\mu} \backslash\{0\}$ is such that $\mu\left(h_{\alpha}\right) \in \mathbb{Z}_{\leq 0}$, then $\left(x_{\alpha}^{+}\right)^{\left(-\mu\left(h_{\alpha}\right)\right)} v \neq 0$. In positive characteristic this stronger statement is not true for all finite-dimensional representations.

The next lemma can be proved exactly as in [Naoi 2012, Lemma 4.5].

Lemma 3.1.5. Let $m_{i} \in \mathbb{Z}_{\geq 0}, i \in I, V$ be a finite-dimensional $U_{\mathbb{F}}\left(\mathfrak{n}^{-}\right)$-module, and suppose $v \in V$ satisfies $\left(x_{i}^{-}\right)^{(k)} v=0$ for all $i \in I, k>m_{i}$. Then, given $\alpha \in R^{+}$, we have $\left(x_{\alpha}^{-}\right)^{(k)} v=0$ for all $k>\sum_{i \in I} n_{i} m_{i}$ where $n_{i}$ are such that $h_{\alpha}=\sum_{i \in I} n_{i} h_{i}$.

3.2. Modules for hyper loop algebras. We now recall some basic results about the category of finite-dimensional $U_{\mathbb{F}}(\tilde{\mathfrak{g}})$-modules in the same spirit as Section 3.1. The results of this subsection can be found in [Jakelić and Moura 2007, Section 3] and references therein.

Given a $U_{\mathbb{F}}(\tilde{\mathfrak{g}})$-module $V$ and $\xi \in U_{\mathbb{F}}(\tilde{\mathfrak{h}})^{*}$, let

$V_{\xi}=\left\{v \in V:\right.$ for all $x \in U_{\mathbb{F}}(\tilde{\mathfrak{h}})$, there exists $k>0$ such that $\left.(x-\xi(x))^{k} v=0\right\}$. 
We say that $V$ is an $\ell$-weight module if $V=\bigoplus V_{\omega}$. In this case, regarding $V$ as a $U_{\mathbb{F}}(\mathfrak{g})$-module, we have

$$
V_{\mu}=\bigoplus_{\substack{\omega \in \mathscr{P}_{\mathbb{F}}: \\ \operatorname{wt}(\boldsymbol{\omega})=\mu}} V_{\omega} \text { for all } \mu \in P \quad \text { and } \quad V=\bigoplus_{\mu \in P} V_{\mu}
$$

A nonzero element of $V_{\omega}$ is said to be an $\ell$-weight vector of $\ell$-weight $\omega$. An $\ell$-weight vector $v$ is said to be a highest- $\ell$-weight vector if $U_{\mathbb{F}}(\tilde{\mathfrak{h}}) v=\mathbb{F} v$ and $\left(x_{\alpha, r}^{+}\right)^{(k)} v=0$ for all $\alpha \in R^{+}$and all $r, k \in \mathbb{Z}, k>0$. If $V$ is generated by a highest- $\ell$-weight vector of $\ell$-weight $\omega, V$ is said to be a highest- $\ell$-weight module of highest $\ell$-weight $\omega$.

Theorem 3.2.1. Let $V$ be a $U_{\mathbb{F}}(\tilde{\mathfrak{g}})$-module.

(a) If $V$ is finite-dimensional, then $V$ is an $\ell$-weight module. Moreover, if $V$ is finite-dimensional and irreducible, then $V$ is a highest- $\ell$-weight module whose highest $\ell$-weight lies in $\mathscr{P}_{\mathbb{F}}^{+}$.

(b) If $V$ is a highest- $\ell$-weight module of highest $\ell$-weight $\omega \in \mathscr{P}_{\mathbb{F}}^{+}$, then $\operatorname{dim} V_{\omega}=1$ and $V_{\mu} \neq 0$ only if $\mu \leq \mathrm{wt}(\omega)$. Moreover, $V$ has a unique maximal proper submodule and, hence, also a unique irreducible quotient. In particular, $V$ is indecomposable.

(c) For each $\omega \in \mathscr{P}_{\mathbb{F}}^{+}$, the local Weyl module $W_{\mathbb{F}}(\omega)$ is nonzero and has finite dimension. Moreover, every finite-dimensional highest- $\ell$-weight module of highest $\ell$-weight $\omega$ is a quotient of $W_{\mathbb{F}}(\omega)$.

(d) If $V$ is finite-dimensional and irreducible, then there exists a unique $\omega \in \mathscr{P}_{\mathbb{F}}^{+}$ such that $V$ is isomorphic to the irreducible quotient $V_{\mathbb{F}}(\omega)$ of $W_{\mathbb{F}}(\omega)$.

(e) For $\mu \in P$ and $\omega \in \mathscr{P}_{\mathbb{F}}^{+}, \mu$ is in $\operatorname{wt}\left(W_{\mathbb{F}}(\omega)\right)$ if and only if $\mu \in \operatorname{wt}\left(W_{\mathbb{F}}(\operatorname{wt}(\omega))\right)$, or equivalently if $w \mu \leq \mathrm{wt}(\omega)$ for all $w \in \mathscr{W}$.

3.3. Graded modules for hyper current algebras. Recall the following elementary fact.

Lemma 3.3.1. Let $A$ be a ring, $I \subset A$ a left ideal, $B=\mathbb{F} \otimes_{\mathbb{Z}} A$ an $\mathbb{F}$-algebra, and $J$ the image of $I$ in $B$, that is, $J$ is the $\mathbb{F}$-span of $\{(1 \otimes a) \in B: a \in I\}$. Then $\mathbb{F} \otimes_{\mathbb{Z}}(A / I)$ is a left $B$-module, $J$ is a left ideal of $B$, and we have an isomorphism of left $B$-modules $B / J \cong \mathbb{F} \otimes_{\mathbb{Z}}(A / I)$.

We shall use Lemma 3.3.1 with $A$ being one of the integral forms so that $B$ is the corresponding hyperalgebra.

Given $\lambda \in P^{+}$, let $I_{\mathbb{Z}}^{c}(\lambda) \subset U_{\mathbb{Z}}(\mathfrak{g}[t])$ be the left ideal generated by

$$
U_{\mathbb{Z}}\left(\mathfrak{n}^{+}[t]\right)^{0}, \quad U_{\mathbb{Z}}\left(\mathfrak{h}[t]_{+}\right)^{0}, \quad h-\lambda(h), \quad\left(x_{\alpha}^{-}\right)^{(k)},
$$

for all $h \in U_{\mathbb{Z}}(\mathfrak{h}), \alpha \in R^{+}, k>\lambda\left(h_{\alpha}\right)$, and set

$$
W_{\mathbb{Z}}^{c}(\lambda)=U_{\mathbb{Z}}(\mathfrak{g}[t]) / I_{\mathbb{Z}}^{c}(\lambda) .
$$


Similarly, if $\ell \geq 0$ is also given, let $I_{\mathbb{Z}}(\ell, \lambda)$ be the left ideal of $U_{\mathbb{Z}}(\mathfrak{g}[t])$ generated by

$$
\begin{gathered}
U_{\mathbb{Z}}\left(\mathfrak{n}^{+}[t]\right)^{0}, \quad U_{\mathbb{Z}}\left(\mathfrak{h}[t]_{+}\right)^{0}, \quad h-\lambda(h), \quad\left(x_{\alpha, s}^{-}\right)^{(k)}, \\
\text { for all } h \in U_{\mathbb{Z}}(\mathfrak{h}), \alpha \in R^{+}, s, k \in \mathbb{Z}_{\geq 0}, k>\max \left\{0, \lambda\left(h_{\alpha}\right)-r_{\alpha}^{\vee} \ell s\right\} .
\end{gathered}
$$

Then set

$$
D_{\mathbb{Z}}(\ell, \lambda)=U_{\mathbb{Z}}(\mathfrak{g}[t]) / I_{\mathbb{Z}}(\ell, \lambda) .
$$

Notice that $W_{\mathbb{Z}}^{c}(\lambda)$ and $D_{\mathbb{Z}}(\ell, \lambda)$ are weight modules.

Since the ideals defining $W_{\mathbb{F}}^{c}(\lambda)$ and $D_{\mathbb{F}}(\ell, \lambda)$ (see Section 1.5) are the images of $I_{\mathbb{Z}}^{c}(\lambda)$ and $I_{\mathbb{Z}}(\ell, \lambda)$ in $U_{\mathbb{F}}(\mathfrak{g}[t])$, respectively, an application of Lemma 3.3.1 gives isomorphisms of $U_{\mathbb{F}}(\mathfrak{g}[t])$-modules

$$
W_{\mathbb{F}}^{c}(\lambda) \cong \mathbb{F} \otimes_{\mathbb{Z}} W_{\mathbb{Z}}^{c}(\lambda) \quad \text { and } \quad D_{\mathbb{F}}(\ell, \lambda) \cong \mathbb{F} \otimes_{\mathbb{Z}} D_{\mathbb{Z}}(\ell, \lambda) .
$$

As before, $D_{\mathbb{Z}}(\ell, \lambda)$ is a quotient of $W_{\mathbb{Z}}^{c}(\lambda)$ for all $\lambda \in P^{+}$and all $\ell>0$. We shall see next (Proposition 3.3.2) that the latter is a finitely generated $\mathbb{Z}$-module and, hence, so is the former. Together with Corollary 1.5.3, this implies that

$$
D_{\mathbb{Z}}(\ell, \lambda) \text { is a free } \mathbb{Z} \text {-module. }
$$

The proof of the next proposition is an adaptation of that of [Jakelic and Moura 2007, Theorem 3.11]. The extra details can be found in [Macedo 2013].

Proposition 3.3.2. For every $\lambda \in P^{+}$, the $U_{\mathbb{Z}}(\mathfrak{g}[t])$-module $W_{\mathbb{Z}}^{c}(\lambda)$ is a finitely generated $\mathbb{Z}$-module.

We now prove an analogue of Theorem 1.5.4 for graded local Weyl modules.

Corollary 3.3.3. Let $\lambda \in P^{+}$and $v$ be the image of 1 in $W_{\mathbb{C}}^{c}(\lambda)$. Then $U_{\mathbb{Z}}(\mathfrak{g}[t]) v$ is a free $\mathbb{Z}$-module of rank $\operatorname{dim}\left(W_{\mathbb{C}}^{c}(\lambda)\right)$. Moreover, $U_{\mathbb{Z}}(\mathfrak{g}[t]) v=\bigoplus_{\mu \in P}\left(U_{\mathbb{Z}}(\mathfrak{g}[t]) v \cap\right.$ $\left.W_{\mathbb{C}}^{c}(\lambda)_{\mu}\right)$. In particular, $U_{\mathbb{Z}}(\mathfrak{g}[t]) v$ is an integral form for $W_{\mathbb{C}}^{c}(\lambda)$.

Proof. To simplify notation, set $L=U_{\mathbb{Z}}\left(\mathfrak{n}^{-}\right) v$. Let also $\vartheta$ be as in the proof of Proposition 3.3.2. Since $v$ satisfies the relations satisfied by $v$, it follows that there exists an epimorphism of $U_{\mathbb{Z}}(\mathfrak{g}[t])$-modules $W_{\mathbb{Z}}(\lambda) \rightarrow L, \vartheta \mapsto v$. Since $W_{\mathbb{Z}}(\lambda)$ is finitely generated, it follows that so is $L$. On the other hand, since $L \subseteq W_{\mathbb{C}}^{c}(\lambda)$, it is also torsion free and, hence, a free $\mathbb{Z}$-module of finite rank. Since $U_{\mathbb{Z}}\left(\mathfrak{n}^{-}\right)$spans $U\left(\mathfrak{n}^{-}\right)$and $W_{\mathbb{C}}^{c}(\lambda)=U\left(\mathfrak{n}^{-}\right) v$, it follows that $L$ contains a basis of $W_{\mathbb{C}}^{c}(\lambda)$. This implies that the rank of $L$ is at least $\operatorname{dim}\left(W_{\mathbb{C}}^{c}(\lambda)\right)$. On the other hand, $\mathbb{C} \otimes_{\mathbb{Z}} L$ is a $\mathfrak{g}[t]$-module generated by the vector $1 \otimes v$ which satisfies the relations (1.5.1). Therefore, it is a quotient of $W_{\mathbb{C}}^{c}(\lambda)$. Since $\operatorname{dim}\left(\mathbb{C} \otimes_{\mathbb{Z}} L\right)=\operatorname{rank}(L)$, the first and the last statements follow. The second statement is clear since $L$ is obviously a weight module. 
Consider the category $\mathscr{G}_{\mathbb{F}}$ of $\mathbb{Z}$-graded finite-dimensional representations of $U_{\mathbb{F}}(\mathfrak{g}[t])$. Recall the functors $\tau_{m}$ defined in the paragraph preceding Remark 1.5.1. For each $U_{\mathbb{F}}(\mathfrak{g})$-module $V$, let $\operatorname{ev}_{0}(V)$ be the module in $\varphi_{\mathbb{F}}$ obtained by extending the action of $U_{\mathbb{F}}(\mathfrak{g})$ to one of $U_{\mathbb{F}}(\mathfrak{g}[t])$ on $V$ by setting $U_{\mathbb{F}}\left(\mathfrak{g}[t]_{+}\right) V=0$. For $\lambda \in P^{+}, r \in \mathbb{Z}$, set $V_{\mathbb{F}}(\lambda, r)=\mathrm{ev}_{r}\left(V_{\mathbb{F}}(\lambda)\right)$ where $\mathrm{ev}_{r}=\tau_{r} \circ \mathrm{ev}_{0}$.

Theorem 3.3.4. Let $\lambda \in P^{+}$.

(a) If $V \in \mathscr{G}_{\mathbb{F}}$ is simple, then it is isomorphic to $V_{\mathbb{F}}(\lambda, r)$ for unique $(\lambda, r) \in P^{+} \times \mathbb{Z}$.

(b) $W_{\mathbb{F}}^{c}(\lambda)$ is finite-dimensional.

(c) If $V$ is a graded finite-dimensional $U_{\mathbb{F}}(\mathfrak{g}[t])$-module generated by a weight vector $v$ of weight $\lambda$ satisfying $U_{\mathbb{F}}\left(\mathfrak{n}^{+}[t]\right)^{0} v=U_{\mathbb{F}}\left(\mathfrak{h}[t]_{+}\right)^{0} v=0$, then $V$ is a quotient of $W_{\mathbb{F}}^{c}(\lambda)$.

Proof. To prove part (a), suppose $V \in \mathscr{G}_{\mathbb{F}}$ is simple. If $V[r], V[s] \neq 0$ for $s<r \in \mathbb{Z}$, $\left(\bigoplus_{k \geq r} V[k]\right)$ would be a proper submodule of $V$, contradicting the fact that it is simple. Thus there must exist a unique $r \in \mathbb{Z}$ such that $V[r] \neq 0$. Since $U_{\mathbb{F}}\left(\mathfrak{g}[t]_{+}\right)$ changes degrees, $V=V[r]$ must be a simple $U_{\mathbb{F}}(\mathfrak{g})$-module. This shows that $V \cong V_{\mathbb{E}}(\lambda, r)$ for some $\lambda \in P^{+}, r \in \mathbb{Z}$.

To prove part (b), observe that $W_{\mathbb{F}}^{c}(\lambda) \cong \mathbb{F} \otimes_{\mathbb{Z}} W_{\mathbb{Z}}^{c}(\lambda)$ (see Lemma 3.3.1). Thus the dimension of $W_{\mathbb{F}}^{c}(\lambda)$ must be at most the number of generators of $W_{\mathbb{Z}}^{c}(\lambda)$, which is proved to be finite in Proposition 3.3.2.

To prove part (c), observe that the $U_{\mathbb{F}}(\mathfrak{g})$-submodule $V^{\prime}=U_{\mathbb{F}}(\mathfrak{g}) v \subseteq V$ is a finitedimensional highest-weight module of highest weight $\lambda$. Thus, by Theorem 3.1.1(c), $V^{\prime}$ is a quotient of $W_{\mathbb{E}}(\lambda)$. The statement follows by comparing the defining relations of $V$ and $W_{\mathbb{F}}^{c}(\lambda)$.

Remark 3.3.5. Denote by $v$ the image of 1 in $W_{\mathbb{F}}^{c}(\lambda)$. From the defining relations (1.5.1) it follows that $\mathbb{E} \otimes_{\mathbb{Z}} U_{\mathbb{Z}}(\mathfrak{g}[t]) v$ is a quotient of $W_{\mathbb{F}}^{c}(\lambda)$. It follows from Theorem 1.5.2(b) that $\mathbb{F} \otimes_{\mathbb{Z}} U_{\mathbb{Z}}(\mathfrak{g}[t]) v \cong W_{\mathbb{F}}^{c}(\lambda)$ for all $\lambda \in P^{+}$(see Section 3.4 below). Moreover, since $\mathbb{E} \otimes_{\mathbb{Z}} W_{\mathbb{Z}}(\lambda) \cong W_{\mathbb{F}}^{c}(\lambda)$, Theorem 1.5.2(b) also implies that $W_{\mathbb{Z}}(\lambda)$ is free.

3.4. Proof of (1.5.4). The argument of the proof will use Corollary 1.5.3, the characteristic-zero version of parts (c) and (d) of Theorem 1.5.2, and the following proposition.

Proposition 3.4.1 [Naoi 2012, Corollary A]. Let $\lambda \in P^{+}$. Then $\operatorname{dim} W_{\mathbb{C}}^{c}(\lambda)=$ $\prod_{i \in I}\left(\operatorname{dim} W_{\mathbb{C}}^{c}\left(\omega_{i}\right)\right)^{\lambda\left(h_{i}\right)}$.

We shall also need the following general construction. Given a $\mathbb{Z}_{s \geq 0}$-filtered $U_{\mathbb{F}}(\mathfrak{g}[t])$-module $W$, we can consider the associated $U_{\mathbb{F}}(\mathfrak{g}[t])$-module $\operatorname{gr}(W)=$ $\bigoplus_{s \geq 0} W_{s} / W_{s-1}$, which obviously has the same dimension as $W$. Suppose now that $W$ is any cyclic $U_{\mathbb{F}}(\mathfrak{g}[t])$-module and fix a generator $w$. Then the $\mathbb{Z}$-grading 
on $U_{\mathbb{F}}(\mathfrak{g}[t])$ induces a filtration on $W$. Namely, set $w$ to have degree zero and define the $s$-th filtered piece of $W$ by $W_{s}=F^{s} U_{\mathbb{F}}(\mathfrak{g}[t]) w$ where $F^{s} U_{\mathbb{F}}(\mathfrak{g}[t])=$ $\bigoplus_{r \leq s} U_{\mathbb{F}}(\mathfrak{g}[t])[r]$. Then $\operatorname{gr}(W)$ is cyclic since it is generated by the image of $w$ in $\operatorname{gr}(W)$.

Recall the notation fixed for (1.5.4): $\omega \in \mathscr{P}_{A}^{\times}, \lambda=\operatorname{wt}(\omega), \varpi$ is the image of $\omega$ in $\mathscr{P}_{\mathbb{F}}$. Also recall that, using (1.5.3), (1.5.4) will be proved if we show that

$$
\operatorname{dim} W_{\mathbb{E}}(\varpi) \leq \operatorname{dim} W_{\mathbb{K}}(\boldsymbol{\omega}) .
$$

Fix $w \in W_{\mathbb{F}}(\varpi)_{\lambda} \backslash\{0\}$. Not only does $w$ generate $W_{\mathbb{F}}(\varpi)$ as a $U_{\mathbb{F}}(\tilde{\mathfrak{g}})$-module, but it also follows from the proof of [Jakelić and Moura 2007, Theorem 3.11] (with a correction incorporated in the proof of [Jakelić and Moura 2010, Theorem 3.7]) that $U_{\mathbb{F}}\left(\mathfrak{n}^{-}[t]\right) w=W_{\mathbb{F}}(\varpi)$. Hence, we can apply the general construction reviewed above to $W_{\mathbb{F}}(\varpi)$. Set $V=\operatorname{gr}\left(W_{\mathbb{F}}(\varpi)\right)$ and denote the image of $w$ in $V$ by $v$. The module $V$ is finite-dimensional and $v$ is a highest-weight vector of weight $\lambda$ satisfying $U_{\mathbb{E}}\left(\mathfrak{h}[t]_{+}\right)^{0} v=0$ (the latter follows since $\operatorname{dim}\left(V_{\lambda}\right)=1, V$ is graded, and $U_{\mathbb{F}}(\mathfrak{h}[t])$ is commutative). Hence, $v$ satisfies the defining relations $(1.5 .1)$ of $W_{\mathbb{F}}^{c}(\lambda)$. In particular,

$$
\operatorname{dim} W_{\mathbb{F}}(\varpi) \leq \operatorname{dim} W_{\mathbb{F}}^{c}(\lambda) .
$$

Since $\operatorname{dim} W_{\mathbb{F}}^{c}(\lambda)=\operatorname{dim} W_{\mathbb{K}}^{c}(\lambda)$ by Corollary 1.5.3, it now suffices to show that

$$
\operatorname{dim} W_{\mathbb{K}}^{c}(\lambda)=\operatorname{dim} W_{\mathbb{K}}(\boldsymbol{\omega}) .
$$

For proving this, consider the decomposition of $\omega$ of the form

$$
\boldsymbol{\omega}=\prod_{j=1}^{m} \boldsymbol{\omega}_{\lambda_{j}, a_{j}}
$$

for some $m \geq 0, a_{j} \in \mathbb{K}^{\times}, a_{i} \neq a_{j}$ for $i \neq j, \lambda_{j} \in P^{+}$such that $\lambda=\sum_{j=1}^{m} \lambda_{j}$. By Theorem 1.5.2(d), in characteristic zero, $W_{\mathbb{K}}(\boldsymbol{\omega}) \cong \otimes_{j=1}^{m} W_{\mathbb{K}}\left(\boldsymbol{\omega}_{\lambda_{j}, a_{j}}\right)$. In characteristic zero, Theorem 1.5.2(c) implies that $\operatorname{dim} W_{\mathbb{K}}\left(\boldsymbol{\omega}_{\lambda_{j}, a_{j}}\right)=\operatorname{dim} W_{\mathbb{K}}^{c}\left(\lambda_{j}\right)$. Hence,

$$
\begin{aligned}
\operatorname{dim} W_{\mathbb{K}}(\boldsymbol{\omega})=\prod_{j=1}^{m} \operatorname{dim} W_{\mathbb{K}}^{c}\left(\lambda_{j}\right)=\prod_{j=1}^{m} \prod_{i \in I} \operatorname{dim} W_{\mathbb{K}}^{c}\left(\omega_{i}\right)^{\lambda_{j}\left(h_{i}\right)} \\
=\prod_{i \in I} W_{\mathbb{K}}^{c}\left(\omega_{i}\right)^{\lambda\left(h_{i}\right)}=\operatorname{dim} W_{\mathbb{K}}^{c}(\lambda) .
\end{aligned}
$$

Here, the second and last equality follow from Proposition 3.4.1 and the others are clear. This completes the proof of (1.5.4).

Notice that all equalities of dimensions proved here actually imply the corresponding equalities of characters. In particular, it follows that

$$
\operatorname{ch}\left(W_{\mathbb{F}}(\varpi)\right)=\prod_{i \in I}\left(\operatorname{ch}\left(W_{\mathbb{C}}^{c}\left(\omega_{i}\right)\right)\right)^{\operatorname{wt}(\varpi)\left(h_{i}\right)} \text { for all } \varpi \in \mathscr{P}_{\mathbb{F}}^{+} .
$$


3.5. Joseph-Mathieu-Polo relations for Demazure modules. We now explain the reason we call the module $D_{\mathbb{F}}(\ell, \lambda)$ a Demazure module. We begin with the following lemma. Let $\gamma$ be the Dynkin diagram automorphism of $\mathfrak{g}$ induced by $w_{0}$ and recall from Section 2.2 that it induces an automorphism of $U_{\mathbb{E}}(\mathfrak{g}[t])$ also denoted by $\gamma$.

Lemma 3.5.1. Let $\lambda \in P^{+}, \ell \geq 0$, and set $\lambda^{*}=-w_{0} \lambda$. Let $W$ be the pull-back of $D_{\mathbb{F}}\left(\ell, \lambda^{*}\right)$ by $\gamma$. Then $D_{\mathbb{F}}(\ell, \lambda) \cong W$.

Proof. Let $v \in D_{\mathbb{F}}\left(\ell, \lambda^{*}\right)_{\lambda^{*}} \backslash\{0\}$. By (1.5.1) and (1.5.2) we have

$$
U_{\mathbb{F}}\left(\mathfrak{n}^{+}[t]\right)^{0} v=U_{\mathbb{F}}\left(\mathfrak{h}[t]_{+}\right)^{0} v=0, \quad h v=\lambda^{*}(h) v, \quad\left(x_{\alpha, s}^{-}\right)^{(k)} v=0,
$$

for all $h \in U_{\mathbb{F}}(\mathfrak{h}), \alpha \in R^{+}, s, k \in \mathbb{Z}_{\geq 0}, k>\max \left\{0, \lambda^{*}\left(h_{\alpha}\right)-s \ell r_{\alpha}^{\vee}\right\}$. Denote by $w$ the vector $v$ regarded as an element of $W$. Evidently, $W=U_{\mathbb{E}}(\mathfrak{g}[t]) w$. Since $\gamma$ restricts to automorphisms of $U_{\mathbb{F}}\left(\mathfrak{n}^{+}[t]\right)$ and of $U_{\mathbb{F}}\left(\mathfrak{h}[t]_{+}\right)$, it follows that $U_{\mathbb{F}}\left(\mathfrak{n}^{+}[t]\right)^{0} w=$ $U_{\mathbb{F}}\left(\mathfrak{h}[t]_{+}\right)^{0} w=0$, while (2.2.5) implies that $w \in W_{\lambda}$. Finally, (2.2.4) and (2.2.5) together imply that

$$
\left(x_{\alpha, s}^{-}\right)^{(k)} w=0 \text { for all } \alpha \in R^{+}, s, k \in \mathbb{Z}_{\geq 0}, k>\max \left\{0, \lambda\left(h_{\alpha}\right)-s \ell r_{\alpha}^{\vee}\right\} .
$$

This shows that $w$ satisfies the defining relations of $D_{\mathbb{F}}(\ell, \lambda)$ and, hence, there exists an epimorphism from $D_{\mathbb{F}}(\ell, \lambda)$ onto $W$. Since $\left(\lambda^{*}\right)^{*}=\lambda$, reversing the roles of $\lambda$ and $\lambda^{*}$ we get an epimorphism on the other direction. Since these are finite-dimensional modules, we are done.

In order to continue, we need the concepts of weight vectors, weight spaces, weight modules and integrable modules for $U_{\mathbb{F}}\left(\hat{\mathfrak{g}}^{\prime}\right)$ which are similar to those for $U_{\mathbb{F}}(\mathfrak{g})$ (see Section 3.1) by replacing $I$ with $\hat{I}$ and $P$ with $\hat{P}^{\prime}$. Also, using the obvious analogue of (2.2.2), we obtain an inclusion $\hat{P}^{\prime} \hookrightarrow U_{\mathbb{F}}\left(\hat{\mathfrak{h}}^{\prime}\right)^{*}$. Let $V$ be a $\mathbb{Z}$-graded $U_{\mathbb{F}}\left(\hat{\mathfrak{g}}^{\prime}\right)$-module whose weights lie in $\hat{P}^{\prime}$. As before, let $V[r]$ denote the $r$-th graded piece of $V$. For $\mu \in \hat{P}$, say $\mu=\mu^{\prime}+m \delta$ with $\mu^{\prime} \in \hat{P}^{\prime}, m \in \mathbb{Z}$, set

$$
V_{\mu}=\left\{v \in V[m]: h v=\mu^{\prime}(h) v \text { for all } h \in U_{\mathbb{F}}\left(\hat{\mathfrak{h}}^{\prime}\right)\right\} .
$$

If $V_{\mu} \neq 0$ we shall say that $\mu$ is a weight of $V$ and let $\operatorname{wt}(V)=\left\{\mu \in \hat{P}: V_{\mu} \neq 0\right\}$.

We record the following partial affine analogue of Theorem 3.1.1.

Theorem 3.5.2. Let $V$ be a graded $U_{\mathbb{F}}\left(\hat{\mathfrak{g}}^{\prime}\right)$-module.

(a) If $V$ is integrable, then $V$ is a weight-module and $\operatorname{wt}(V) \subseteq \hat{P}$. Moreover, $\operatorname{dim} V_{\mu}=\operatorname{dim} V_{\sigma \mu}$ for all $\sigma \in \widehat{\mathscr{W}}, \mu \in \hat{P}$.

(b) If $V$ is a highest-weight module of highest weight $\lambda, \operatorname{dim}\left(V_{\lambda}\right)=1$ and $V_{\mu} \neq 0$ only if $\mu \leq \lambda$. Moreover, $V$ has a unique maximal proper submodule and, hence, also a unique irreducible quotient. In particular, $V$ is indecomposable. 
(c) Let $\Lambda \in \hat{P}^{+}$and $m=\Lambda(d)$. Then the $U_{\mathbb{F}}\left(\hat{\mathfrak{g}}^{\prime}\right)$-module $\hat{W}_{\mathbb{F}}(\Lambda)$ generated by a vector $v$ of degree $m$ satisfying the defining relations

$$
U_{\mathbb{F}}\left(\hat{\mathfrak{n}}^{+}\right)^{0} v=0, \quad h v=\Lambda(h) v \quad \text { and } \quad\left(x_{i}^{-}\right)^{(k)} v=0,
$$

for all $h \in U_{\mathbb{F}}\left(\hat{\mathfrak{h}}^{\prime}\right), i \in \hat{I}, k>\Lambda\left(h_{i}\right)$, is nonzero and integrable. Moreover, for every positive real root $\alpha$, we have

$$
\left(x_{\alpha}^{-}\right)^{(k)} v=0 \text { for all } k>\Lambda\left(h_{\alpha}\right) .
$$

Furthermore, every integrable highest-weight module of highest weight $\Lambda$ is a quotient of $\hat{W}_{\mathbb{F}}(\Lambda)$.

Given $\Lambda \in \hat{P}^{+}, \sigma \in \widehat{\mathscr{W}}$, the Demazure module $V_{\mathbb{F}}^{\sigma}(\Lambda)$ is defined as the $U_{\mathbb{F}}\left(\hat{\mathfrak{b}}^{\prime+}\right)$ submodule generated by $\hat{W}_{\mathbb{E}}(\Lambda)_{\sigma \Lambda}$ (see [Fourier and Littelmann 2007; Mathieu 1989; Naoi 2012]). In particular, $V_{\mathbb{F}}^{\sigma}(\Lambda) \cong V_{\mathbb{F}}^{\sigma^{\prime}}(\Lambda)$ if $\sigma \Lambda=\sigma^{\prime} \Lambda$ for some $\sigma^{\prime} \in \widehat{\mathscr{W}}$. Our focus is on the Demazure modules which are stable under the action of $U_{\mathbb{F}}(\mathfrak{g})$. Since $V_{\mathbb{F}}^{\sigma}(\Lambda)$ is defined as a $U_{\mathbb{F}}\left(\hat{\mathfrak{b}}^{\prime+}\right)$-module, it is stable under the action of $U_{\mathbb{F}}(\mathfrak{g})$ if, and only if,

$$
U_{\mathbb{F}}\left(\mathfrak{n}^{-}\right)^{0} \hat{W}_{\mathbb{F}}(\Lambda)_{\sigma \Lambda}=0
$$

In particular, since $V_{\mathbb{F}}^{\sigma}(\Lambda)$ is an integrable $U_{\mathbb{F}}\left(\mathfrak{s l}_{\alpha}\right)$-module for any $\alpha \in R^{+}$, it follows that $(\sigma \Lambda)\left(h_{\alpha}\right) \leq 0$ for all $\alpha \in R^{+}$. Conversely, using the exchange condition for Coxeter groups (see [Humphreys 1990, Section 5.8]), one easily deduces that, for all $i \in \hat{I}$, we have

$$
\left(x_{i}^{\varepsilon}\right)^{(k)} \hat{W}_{\mathbb{F}}(\Lambda)_{\sigma \Lambda}=0 \text { for all } k>0
$$

where $\varepsilon=+$ if $\sigma \Lambda\left(h_{i}\right) \geq 0$ and $\varepsilon=-$ if $\sigma \Lambda\left(h_{i}\right) \leq 0$. This implies that if $\sigma \Lambda\left(h_{i}\right) \leq 0$ for all $i \in I$, then $V_{\mathbb{F}}^{\sigma}(\Lambda)$ is $U_{\mathbb{F}}(\mathfrak{g})$-stable. Thus, henceforth, assume $(\sigma \Lambda)\left(h_{i}\right) \leq 0$ for all $i \in I$ and observe that this implies that $\sigma \Lambda$ must have the form

$$
\sigma \Lambda=\ell \Lambda_{0}+w_{0} \lambda+m \delta \text { for some } \lambda \in P^{+}, m \in \mathbb{Z} \text {, and } \ell=\Lambda(c) .
$$

Conversely, given $\ell \in \mathbb{Z}_{\geq 0}, \lambda \in P^{+}$, and $m \in \mathbb{Z}$, since $\widehat{\mathscr{W}}$ acts simply transitively on the set of alcoves of $\hat{\mathfrak{h}}^{*}$ (see [Humphreys 1990, Theorem 4.5.(c)]), there exists a unique $\Lambda \in \hat{P}^{+}$such that $\ell \Lambda_{0}+w_{0} \lambda+m \delta \in \widehat{\mathscr{W}} \Lambda$. Thus, if $\sigma \in \widehat{\mathscr{W}}$ and $\Lambda \in \hat{P}^{+}$are such that

$$
\sigma \Lambda=\ell \Lambda_{0}+w_{0} \lambda+m \delta
$$

then $V_{\mathbb{F}}^{\sigma}(\Lambda)$ is $U_{\mathbb{F}}(\mathfrak{g})$-stable. Henceforth, we fix $\sigma, \Lambda, w_{0}, \lambda$, and $m$ as in (3.5.4). Notice that if $\gamma= \pm \alpha+s \delta \in \hat{R}^{+}$with $\alpha \in R^{+}$, then

$$
\sigma \Lambda\left(h_{\gamma}\right)= \pm w_{0} \lambda\left(h_{\alpha}\right)+s \ell r_{\alpha}^{\vee}
$$


The following lemma is a rewriting of [Mathieu 1989, Lemme 26] using the above fixed notation.

Lemma 3.5.3. The $U_{\mathbb{F}}\left(\hat{\mathfrak{b}}^{\prime+}\right)$-module $V_{\mathbb{F}}^{\sigma}(\Lambda)$ is isomorphic to the $U_{\mathbb{F}}\left(\hat{\mathfrak{b}}^{\prime+}\right)$-module generated by a vector $v$ of degree $m$ satisfying the following defining relations: $h v=\sigma \Lambda(h) v, h \in U_{\mathbb{F}}\left(\hat{\mathfrak{h}}^{\prime}\right), U_{\mathbb{F}}\left(\mathfrak{h}[t]_{+}\right)^{0} v=U_{\mathbb{F}}\left(\mathfrak{n}^{-}[t]_{+}\right)^{0} v=0$, and

$$
\left(x_{\alpha, s}^{+}\right)^{(k)} v=0 \text { for all } \alpha \in R^{+}, s \geq 0, k>\max \left\{0,-w_{0} \lambda\left(h_{\alpha}\right)-s \ell r_{\alpha}^{\vee}\right\} \text {. }
$$

Remark 3.5.4. Mathieu [1989] attributes Lemma 3.5.3 to Joseph and Polo. This is the reason for the title of this subsection. The original version of this lemma in [Mathieu 1989] gives generator and relations for any Demazure module, not only for the $U_{\mathbb{F}}(\mathfrak{g})$-stable ones.

The following is the main result of this subsection.

Proposition 3.5.5. The graded $U_{\mathbb{F}}(\mathfrak{g}[t])$-modules $V_{\mathbb{F}}^{\sigma}(\Lambda)$ and $D_{\mathbb{F}}(\ell, \lambda, m)$ are isomorphic.

Proof. It suffices to prove the statement for $m=0$, so for simplicity we assume that this is the case. Proceeding as in [Fourier and Littelmann 2007, Corollary 1] (see also [Naoi 2012, Proposition 3.6]) we show that $V_{\mathbb{F}}^{\sigma}(\Lambda)$ is a quotient of $D_{\mathbb{F}}(\ell, \lambda)$. Namely, let $v$ be a nonzero vector in $\hat{W}_{\mathbb{F}}(\Lambda)_{\mu}$ where $\mu=w_{0} \sigma \Lambda$. Quite clearly $v$ generates $V_{\mathbb{F}}^{\sigma}(\Lambda)$. It follows that $v$ is an extremal weight vector and, hence, satisfies the relations

$$
\left(x_{\gamma}^{ \pm}\right)^{(k)} v=0 \text { for all } k>\max \left\{0, \mp \mu\left(h_{\gamma}\right)\right\}
$$

and all positive real roots $\gamma$. In particular, taking $\gamma=\alpha+s \delta$ with $\alpha \in R^{+}$and $s \geq 0$, it follows that

$$
-\mu\left(h_{\gamma}\right)=-\lambda\left(h_{\alpha}\right)-\ell r_{\alpha}^{\vee} s \leq 0,
$$

showing $\left(x_{\alpha, s}^{+}\right)^{(k)} v=0$ for all $k>0$. Similarly, taking $\gamma=-\alpha+s \delta$, we get

$$
-\mu\left(h_{\gamma}\right)=\lambda\left(h_{\alpha}\right)-\ell r_{\alpha}^{\vee} s,
$$

which shows that $v$ satisfies the relations determined by (1.5.2). It remains to be shown that $U_{\mathbb{F}}\left(\mathfrak{h}[t]_{+}\right)^{0} v=0$. This can be proved as in [Mathieu 1989, Lemme 26]. Alternatively, this can also be shown by proving that there exists a surjective map from $D\left(\ell, \lambda^{*}\right)$ to the pull-back of $V_{\mathbb{F}}^{\sigma}(\Lambda)$ by the automorphism $\psi$ defined in Section 2.2 (similarly to what we do in the next paragraph), and then comparing weights (one uses a vector as in Lemma 3.5.3 to prove the existence of such a map). It now suffices to show that $\operatorname{dim}\left(D_{\mathbb{F}}(\ell, \lambda)\right) \leq \operatorname{dim}\left(V_{\mathbb{F}}^{\sigma}(\Lambda)\right)$.

Now let $v$ be in $D_{\mathbb{F}}\left(\ell, \lambda^{*}\right)_{\lambda^{*}} \backslash\{0\}, W$ be the pull-back of $D_{\mathbb{F}}\left(\ell, \lambda^{*}\right)$ by $\psi$, and $w$ denote $v$ when regarded as an element of $W$. Since $U_{\mathbb{F}}\left(\mathfrak{n}^{+}[t]\right)^{0} v=0$, and since 
(3.5.2) implies that $\psi\left(U_{\mathbb{F}}\left(\mathfrak{n}^{-}[t]\right)^{0}\right)=U_{\mathbb{F}}\left(\mathfrak{n}^{+}[t]\right)^{0}$, it follows that $U_{\mathbb{F}}\left(\mathfrak{n}^{-}[t]\right)^{0} w=0$. Also, $\psi$ restricts to an automorphism of $U_{\mathbb{F}}\left(\mathfrak{h}[t]_{+}\right)$and, hence, $U_{\mathbb{F}}\left(\mathfrak{h}[t]_{+}\right)^{0} w=0$. Since $h v=\lambda^{*}(h) v$ for all $h \in U_{\mathbb{F}}(\mathfrak{h}),(2.2 .3)$ implies that $h w=w_{0} \lambda(h) w$ for all $h \in U_{\mathbb{F}}(\mathfrak{h})$. Finally, the defining relations of $v$ and (2.2.1) imply that

$$
\left(x_{\alpha, s}^{+}\right)^{(k)} w=\left(x_{\alpha, s}^{-}\right)^{(k)} v=0 \text { for all } \alpha \in R^{+}, s \geq 0, k>\max \left\{0, \lambda^{*}\left(h_{\alpha}\right)-s \ell r_{\alpha}^{\vee}\right\} .
$$

Thus $w$ satisfies all the defining relations of $V_{\mathbb{F}}^{\sigma}(\Lambda)$ in Lemma 3.5.3. Hence, $W$ is a quotient of $V_{\mathbb{F}}^{\sigma}(\Lambda)$ and therefore $\operatorname{dim}(W) \leq \operatorname{dim}\left(V_{\mathbb{F}}^{\sigma}(\Lambda)\right)$. Since $\operatorname{dim}\left(D_{\mathbb{F}}\left(\ell, \lambda^{*}\right)\right)=$ $\operatorname{dim}\left(D_{\mathbb{F}}(\ell, \lambda)\right)$ by Lemma 3.5.1, we are done.

Corollary 3.5.6. $D_{\mathbb{F}}(\ell, \lambda)$ is isomorphic to the quotient of $U_{\mathbb{F}}(\mathfrak{g}[t])$ by the left ideal $I_{\mathbb{F}}^{-}(\ell, \lambda)$ generated by $h-w_{0} \lambda(h), h \in U_{\mathbb{F}}(\mathfrak{h}), U_{\mathbb{F}}\left(\mathfrak{h}[t]_{+}\right)^{0}, U_{\mathbb{F}}\left(\mathfrak{n}^{-}[t]\right)^{0}$, and

$$
\left(x_{\alpha, s}^{+}\right)^{(k)} \text { for all } \alpha \in R^{+}, s \geq 0, k>\max \left\{0,-w_{0} \lambda\left(h_{\alpha}\right)-s \ell r_{\alpha}^{\vee}\right\} \text {. }
$$

Remark 3.5.7. Observe that the difference between our first definition of $D_{\mathbb{F}}(\ell, \lambda)$ and the one given by Corollary 3.5.6 lies on exchanging a "highest-weight generator" by a "lowest-weight" one. More precisely, let $v$ be as in Lemma 3.5.3. Then the isomorphism of Proposition 3.5.5 must send $v$ to a nonzero element in $D_{\mathbb{E}}(\ell, \lambda)_{w_{0} \lambda}$. In particular, if $w$ is in $D_{\mathbb{E}}(\ell, \lambda)_{w_{0} \lambda}$, it satisfies the relations listed in Lemma 3.5.3. The second part of our proof of Proposition 3.5.5 differs from the one given in [Fourier and Littelmann 2007, Corollary 1] in characteristic zero. It is claimed there that a vector in $D_{\mathbb{F}}(\ell, \lambda)_{w_{0} \lambda}$ must satisfy several relations, including (3.5.5), without further justification. Proposition 3.5.5 implies that this is true, but we do not see how to deduce it so directly (even in characteristic zero) since we cannot use extremal-weight vector theory to such vectors $D_{\mathbb{F}}(\ell, \lambda)$ a priori contained in an integrable module for the full affine hyperalgebra.

Corollary 3.5.8. Let $\mathfrak{g}=\mathfrak{s l}_{2}$ and consider the subalgebra $\mathfrak{a}=\mathfrak{n}^{-}[t] \oplus \mathfrak{h}[t] \oplus$ $\mathfrak{n}^{+}[t]_{+} \subseteq \mathfrak{g}[t]$. For $\ell, \lambda \in \mathbb{Z}_{\geq 0}$, let $I_{\mathbb{F}}^{\prime}(\ell, \lambda)$ be the left ideal of $U_{\mathbb{F}}(\mathfrak{a})$ generated by the generators of $I_{\mathbb{F}}(\ell, \lambda)$ which lie in $U_{\mathbb{F}}(\mathfrak{a})$. Then, given $k, l, s \in \mathbb{Z}_{\geq 0}$ with $k>\max \{0, \lambda-s \ell\}$, we have

$$
\left(x_{i}^{+}\right)^{(l)}\left(x_{i, s}^{-}\right)^{(k)} \in U_{\mathbb{F}}(\mathfrak{a}) U_{\mathbb{F}}\left(\mathfrak{n}^{+}\right)^{0} \oplus I_{\mathbb{F}}^{\prime}(\ell, \lambda)
$$

where $i$ is the unique element of $I$.

Proof. The statement is a hyperalgebraic version of [Naoi 2012, Lemma 4.10] and the proof follows a similar outline. Namely, by using the automorphism of $\mathfrak{g}[t]$ determined by $x_{i, r}^{ \pm} \mapsto x_{i, r}^{\mp}, i \in I, r \in \mathbb{Z}_{\geq 0}$, we observe that (3.5.7) is equivalent to

$$
\left(x_{i}^{-}\right)^{(l)}\left(x_{i, s}^{+}\right)^{(k)} \in U_{\mathbb{F}}\left(\mathfrak{a}^{-}\right) U_{\mathbb{F}}\left(\mathfrak{n}^{-}\right)^{0}+I_{\mathbb{F}}^{\prime \prime}(\ell, \lambda)
$$

for all $k, l, s \in \mathbb{Z}_{\geq 0}, k>\max \{0, \lambda-s \ell\}$, where $\mathfrak{a}^{-}=\mathfrak{n}^{-}[t]_{+} \oplus \mathfrak{h}[t] \oplus \mathfrak{n}^{+}[t]$ and $I_{\mathbb{F}}^{\prime \prime}(\ell, \lambda)$ is the left ideal of $U_{\mathbb{F}}\left(\mathfrak{a}^{-}\right)$generated by the generators of $I_{\mathbb{F}}^{-}(\ell, \lambda)$ given 
in Corollary 3.5.6 which lie in $U_{\mathbb{F}}\left(\mathfrak{a}^{-}\right)$. Since $\mathfrak{g}[t]=\mathfrak{a}^{-} \oplus \mathfrak{n}^{-}$, the PBW theorem implies that

$$
U_{\mathbb{F}}(\mathfrak{g}[t])=U_{\mathbb{F}}\left(\mathfrak{a}^{-}\right) U_{\mathbb{F}}\left(\mathfrak{n}^{-}\right)^{0} \oplus U_{\mathbb{F}}\left(\mathfrak{a}^{-}\right),
$$

and, hence, $\left(x_{i}^{-}\right)^{(l)}\left(x_{i, s}^{+}\right)^{(k)}=u+u^{\prime}$ with $u \in U_{\mathbb{F}}\left(\mathfrak{a}^{-}\right) U_{\mathbb{F}}\left(\mathfrak{n}^{-}\right)^{0}$ and $u^{\prime} \in U_{\mathbb{F}}\left(\mathfrak{a}^{-}\right)$. Consider the Demazure module $D_{\mathbb{F}}(\ell, \lambda)$ and let $w \in D_{\mathbb{F}}(\ell, \lambda)_{-\lambda} \backslash\{0\}$. It follows from the proof of Proposition 3.5.5 that if $k>\max \{0, \lambda-s \ell\}$, then

$$
u^{\prime} w=\left(\left(x_{i}^{-}\right)^{(l)}\left(x_{i, s}^{+}\right)^{(k)}-u\right) w=0 .
$$

Since $\hat{\mathfrak{b}}^{\prime+}=\mathfrak{a}^{-} \oplus \mathbb{C} c$ and $\mathfrak{a}^{-}$is an ideal of $\hat{\mathfrak{b}}^{\prime+}$, it follows from Lemma 3.5.3 that $I_{\mathbb{F}}^{\prime \prime}(\ell, \lambda)$ is the annihilating ideal of $w$ inside $U_{\mathbb{F}}(\mathfrak{a})$, and, hence, $u^{\prime} \in I_{\mathbb{F}}^{\prime \prime}(\ell, \lambda)$.

\section{Joseph's Demazure flags}

4.1. Quantum groups. Let $\mathbb{C}(q)$ be the field of rational functions on an indeterminate $q$. Let also $C=\left(c_{i j}\right)_{i, j \in \hat{I}}$ be the Cartan matrix of $\hat{\mathfrak{g}}$, and $d_{i}$, with $i \in \hat{I}$, be nonnegative relatively prime integers such that the matrix $D C$, with $D=\operatorname{diag}\left(d_{i}\right)_{i \in I}$, is symmetric. Set $q_{i}=q^{d_{i}}$ and for $m, n \in \mathbb{Z}, n \geq 0$, set

$$
\begin{gathered}
{[m]_{q_{i}}=\frac{q_{i}^{m}-q_{i}^{-m}}{q_{i}-q_{i}^{-1}}, \quad[n]_{q_{i}} !=[n]_{q_{i}}[n-1]_{q_{i}} \cdots[1]_{q_{i}},} \\
{\left[\begin{array}{c}
m \\
n
\end{array}\right]_{q_{i}}=\frac{[m]_{q_{i}}[m-1]_{q_{i}} \ldots[m-n+1]_{q_{i}}}{[n]_{q_{i}} !} .}
\end{gathered}
$$

The quantum group $U_{q}\left(\hat{\mathfrak{g}}^{\prime}\right)$ is a $\mathbb{C}(q)$-associative algebra (with 1$)$ with generators $x_{i}^{ \pm}, k_{i}^{ \pm 1}, i \in \hat{I}$ subject to the following defining relations for all $i, j \in \hat{I}$ :

$$
\begin{aligned}
k_{i} k_{i}^{-1}= & 1, \quad k_{i} k_{j}=k_{j} k_{i}, \quad k_{i} x_{j}^{ \pm} k_{i}^{-1}=q_{i}^{ \pm c_{i j}} x_{j}^{ \pm}, \quad\left[x_{i}^{+}, x_{j}^{-}\right]=\delta_{i j} \frac{k_{i}-k_{i}^{-1}}{q_{i}-q_{i}^{-1}}, \\
& \sum_{m=0}^{1-c_{i j}}(-1)^{m}\left[\begin{array}{c}
1-c_{i j} \\
m
\end{array}\right]_{q_{i}}\left(x_{i}^{ \pm}\right)^{1-c_{i j}-m} x_{j}^{ \pm}\left(x_{i}^{ \pm}\right)^{m}=0, \quad i \neq j .
\end{aligned}
$$

Let $U_{q}\left(\hat{\mathfrak{n}}^{ \pm}\right)$be the subalgebra generated by $x_{i}^{ \pm}, i \in \hat{I}$, and $U_{q}\left(\hat{\mathfrak{b}}^{ \pm}\right)$be the subalgebra generated by $U_{q}\left(\hat{\mathfrak{n}}^{ \pm}\right)$together with $k_{i}^{ \pm 1}, i \in \hat{I}$.

We shall need an integral form of $U\left(\hat{\mathfrak{g}}^{\prime}\right)$. Let $\mathbb{Z}_{q}=\mathbb{Z}\left[q, q^{-1}\right], U_{\mathbb{Z}_{q}}\left(\hat{\mathfrak{n}}^{ \pm}\right)$be the $\mathbb{Z}_{q}$-subalgebra of $U_{q}\left(\hat{\mathfrak{n}}^{ \pm}\right)$generated by $\left(x_{i}^{ \pm}\right)^{m} /\left([m]_{q_{i}} !\right), i \in \hat{I}, m \geq 0$, and $U_{\mathbb{Z}_{q}}\left(\hat{\mathfrak{g}}^{\prime}\right)$ be the $\mathbb{Z}_{q}$-subalgebra of $U_{q}\left(\hat{\mathfrak{g}}^{\prime}\right)$ generated by $U_{\mathbb{Z}_{q}}\left(\hat{\mathfrak{n}}^{ \pm}\right)$and $k_{i}, i \in \hat{I}$. Let also $U_{\mathbb{Z}_{q}}\left(\hat{\mathfrak{b}}^{ \pm}\right)=U_{q}\left(\hat{\mathfrak{b}}^{ \pm}\right) \cap U_{\mathbb{Z}_{q}}\left(\hat{\mathfrak{g}}^{\prime}\right)$. Then $U_{\mathbb{Z}_{q}}(\mathfrak{a})$, where $\mathfrak{a}=\hat{\mathfrak{g}}^{\prime}, \hat{\mathfrak{n}}^{ \pm}, \hat{\mathfrak{b}}^{ \pm}$, is a free $\mathbb{Z}_{q^{-}}$ module such that the natural map $\mathbb{C}(q) \otimes_{\mathbb{Z}_{q}} U_{\mathbb{Z}_{q}}(\mathfrak{a}) \rightarrow U_{q}(\mathfrak{a})$ is a $\mathbb{C}(q)$-algebra isomorphism. In other words, $U_{\mathbb{Z}_{q}}(\mathfrak{a})$ is a $\mathbb{Z}_{q}$-form of $U_{q}(\mathfrak{a})$. Moreover, letting $\mathbb{Z}$ be a $\mathbb{Z}_{q}$-module where $q$ acts as 1 , there exists an epimorphism of $\mathbb{Z}$-algebras 
$\mathbb{Z} \otimes_{\mathbb{Z}_{q}} U_{\mathbb{Z}_{q}}(\mathfrak{a}) \rightarrow U_{\mathbb{Z}}(\mathfrak{a})$, which is an isomorphism if $\mathfrak{a}=\hat{\mathfrak{n}}^{ \pm}$, and whose kernel is the ideal generated by $k_{i}-1, i \in \hat{I}$, for $\mathfrak{a}=\hat{\mathfrak{g}}^{\prime}, \hat{\mathfrak{b}}^{ \pm}$.

Given $\Lambda \in \hat{P}^{+}$, let $V_{q}(\Lambda)$ be the simple (type 1) $U_{q}\left(\hat{\mathfrak{g}}^{\prime}\right)$-module of highest weight $\Lambda$. Given a highest-weight vector $v \in V_{q}(\Lambda)$, set $V_{\mathbb{Z}_{q}}(\Lambda)=U_{\mathbb{Z}_{q}}\left(\hat{\mathfrak{n}}^{-}\right) v$, which is a $\mathbb{Z}_{q}$-form of $V_{q}(\Lambda)$. Given $\sigma \in \widehat{\mathscr{W}}$ and a nonzero vector $v \in V_{q}(\Lambda)$ of weight $\sigma \Lambda$, set $V_{\mathbb{Z}_{q}}^{\sigma}(\Lambda)=U_{\mathbb{Z}_{q}}\left(\hat{\mathfrak{n}}^{+}\right) v$, which is a free $\mathbb{Z}_{q}$-module as well as a $U_{\mathbb{Z}_{q}}\left(\hat{\mathfrak{b}}^{+}\right)$-module, and $\mathbb{C} \otimes_{\mathbb{Z}_{q}} V_{\mathbb{Z}_{q}}^{\sigma}(\Lambda) \cong V_{\mathbb{C}}^{\sigma}(\Lambda)$. In particular,

$$
V_{\mathbb{Z}}^{\sigma}(\Lambda):=\mathbb{Z} \otimes_{\mathbb{Z}_{q}} V_{\mathbb{Z}_{q}}^{\sigma}(\Lambda)
$$

is an integral form of $V_{\mathbb{C}}^{\sigma}(\Lambda)$.

4.2. Crystals. A normal crystal associated to the root data of $\hat{\mathfrak{g}}$ defined as a set $B$ equipped with maps $\tilde{e}_{i}, \tilde{f}_{i}: B \rightarrow B \sqcup\{0\}, \varepsilon_{i}, \varphi_{i}: B \rightarrow \mathbb{Z}$, for each $i \in \hat{I}$, and wt $: B \rightarrow \hat{P}$ satisfying

(1) $\varepsilon_{i}(b)=\max \left\{n: \tilde{e}_{i} b \neq 0\right\}, \varphi_{i}(b)=\max \left\{n: \tilde{f}_{i} b \neq 0\right\}$, for all $i \in \hat{I}, b \in B$;

(2) $\varphi_{i}(b)-\varepsilon_{i}(b)=\operatorname{wt}(b)\left(h_{i}\right)$, for all $i \in \hat{I}, b \in B$;

(3) for $b, b^{\prime} \in B, b^{\prime}=\tilde{e}_{i} b$ if and only if $\tilde{f}_{i} b^{\prime}=b$;

(4) if $b \in B$ and $i \in \hat{I}$ are such that $\tilde{e}_{i} b \neq 0$, then $\operatorname{wt}\left(\tilde{e}_{i} b\right)=\operatorname{wt}(b)+\alpha_{i}$.

For convenience, we extend $\tilde{e}_{i}, \tilde{f}_{i}, \varepsilon_{i}, \varphi_{i}$, wt to $B \sqcup\{0\}$ by setting them to map 0 to 0 . Denote by $\mathscr{E}$ the submonoid of the monoid of maps $B \sqcup\{0\} \rightarrow B \sqcup\{0\}$ generated by $\left\{\tilde{e}_{i}: i \in \hat{I}\right\}$, and similarly define $\mathscr{F}_{\text {. }}$. A normal crystal is said to be of highest weight $\Lambda \in \hat{P}^{+}$if there exists $b_{\Lambda} \in B$ satisfying

$$
\operatorname{wt}\left(b_{\Lambda}\right)=\Lambda, \quad \mathscr{E} b_{\Lambda}=\{0\}, \quad \text { and } \quad \mathscr{F} b_{\Lambda}=B .
$$

Given $B^{\prime} \subset B$ and $\mu \in \hat{P}$, define $B_{\mu}^{\prime}=\left\{b \in B^{\prime}: \operatorname{wt}(b)=\mu\right\}$ and define the character of $B^{\prime}$ as $\operatorname{ch}\left(B^{\prime}\right)=\sum_{\mu \in \hat{P}} \# B_{\mu}^{\prime} e^{\mu} \in \mathbb{Z}[\hat{P}]$.

Given crystals $B_{1}$ and $B_{2}$, a morphism from $B_{1}$ to $B_{2}$ is a map $\psi: B_{1} \rightarrow B_{2} \sqcup\{0\}$ satisfying

(1) if $\psi(b) \neq 0$, then $\operatorname{wt}(\psi(b))=\operatorname{wt}(b), \varepsilon_{i}(\psi(b))=\varepsilon_{i}(b), \varphi_{i}(\psi(b))=\varphi_{i}(b)$, for all $i \in \hat{I}$;

(2) if $\tilde{e}_{i} b \neq 0$, then $\psi\left(\tilde{e}_{i} b\right)=\tilde{e}_{i} \psi(b)$;

(3) if $\tilde{f}_{i} b \neq 0$, then $\psi\left(\tilde{f}_{i} b\right)=\tilde{f}_{i} \psi(b)$.

The set $B_{1} \times B_{2}$ admits a structure of crystal denoted by $B_{1} \otimes B_{2}$ (see [Joseph 2003, Section 2.4]). There is, up to isomorphism, exactly one family $\left\{B(\Lambda): \Lambda \in \hat{P}^{+}\right\}$of normal highest-weight crystals such that for all $\lambda, \mu \in \hat{P}^{+}$, the crystal structure of $B(\lambda) \otimes B(\mu)$ induces a crystal structure on its subset $\mathscr{F}\left(b_{\lambda} \otimes b_{\mu}\right)$, the inclusion is a homomorphism of crystals, and $\mathscr{F}\left(b_{\lambda} \otimes b_{\mu}\right) \cong B(\lambda+\mu)$. 
Given a crystal $B$ and $\sigma \in \widehat{\mathscr{W}}$ with a fixed reduced expression $\sigma=s_{i_{1}} \ldots s_{i_{n}}$, define

$$
\mathscr{\mathscr { E }}^{\sigma}=\left\{\tilde{e}_{i_{1}}^{m_{1}} \ldots \tilde{e}_{i_{n}}^{m_{n}}: m_{j} \in \mathbb{N}\right\} \subset \mathscr{E} \quad \text { and } \quad \mathscr{F}^{\sigma}=\left\{\tilde{f}_{i_{1}}^{m_{1}} \ldots \tilde{f}_{i_{n}}^{m_{n}}: m_{j} \in \mathbb{N}\right\} \subset \mathscr{F} .
$$

If $B=B(\Lambda), \Lambda \in \hat{P}^{+}$and $\sigma \in \widehat{\mathcal{W}}$, define the Demazure subset $B^{\sigma}(\Lambda)=\mathscr{F}^{\sigma} b_{\Lambda} \subseteq$ $B(\Lambda)$. Then $B^{\sigma}(\Lambda)$ is $\mathscr{E}$-stable: $\mathscr{E} B^{\sigma}(\Lambda) \subset B^{\sigma}(\Lambda) \sqcup\{0\}$. It was proved in [Joseph 2003, Section 4.6] that $\operatorname{ch}\left(V_{\mathbb{C}}^{\sigma}(\Lambda)\right)=\operatorname{ch}\left(B^{\sigma}(\Lambda)\right)$. This fact and the following theorem are the main results of [Joseph 2003] that we shall need.

Theorem 4.2.1. Let $\Lambda, \mu \in \hat{P}^{+}$. For any $\sigma \in \widehat{\mathscr{W}}$, there exist a finite set $J$ and elements $\sigma_{j} \in \widehat{\mathscr{W}}, b_{j} \in B^{\sigma}(\Lambda)$ for each $j \in J$, satisfying

(1) $b_{\mu} \otimes B^{\sigma}(\Lambda)=\sqcup_{j \in J} B_{j}$ where $B_{j}:=\mathscr{F}^{\sigma_{j}}\left(b_{\mu} \otimes b_{j}\right)$;

(2) $\mathscr{E}\left(b_{\mu} \otimes b_{j}\right)=\{0\}$;

(3) $\operatorname{ch}\left(B_{j}\right)=\operatorname{ch}\left(B^{\sigma_{j}}\left(v_{j}\right)\right)$, where $v_{j}=\mu+\operatorname{wt}\left(b_{j}\right) \in \hat{P}^{+}$.

Remark 4.2.2. The proof of Theorem 4.2.1 establishes an algorithm to find the set $J$ and the elements $\sigma_{j}, b_{j}$.

4.3. Globalizing. The theory of global basis of Kashiwara shows, in particular, that for each $\Lambda \in \hat{P}^{+}$, there is a map $G: B(\Lambda) \rightarrow V_{q}(\Lambda)$ such that

$$
V_{\mathbb{Z}_{q}}(\Lambda)=\bigoplus_{b \in B(\Lambda)} \mathbb{Z}_{q} G(b),
$$

the weight of $G(b)$ is $\mathrm{wt}(b)$ and $G\left(b_{\Lambda}\right)$ is a highest-weight vector of $V_{q}(\Lambda)$.

Fix $\Lambda, \mu \in \hat{P}^{+}, \sigma \in \widehat{\mathscr{W}}$ and let $J, b_{j}, \sigma_{j}, v_{j}, j$ be in $J$, be as in Theorem 4.2.1. Let $b$ be in $B(\Lambda)_{\sigma \Lambda}$ and set $V_{\mathbb{Z}_{q}}^{\sigma}(\Lambda)=U_{\mathbb{Z}_{q}}\left(\hat{\mathfrak{n}}^{+}\right) G(b)$. Similarly, let $b_{j}^{\prime}$ be the unique element of $B_{j}$ such that $\operatorname{wt}\left(b_{j}^{\prime}\right)=\sigma_{j} v_{j}$. Choose a linear order on $J$ such that $\operatorname{wt}\left(b_{j}\right)<\operatorname{wt}\left(b_{k}\right)$ only if $j>k$. For $j \in J$, let $Y_{j}$ be the $\mathbb{Z}_{q}$-submodule of $V_{q}(\mu) \otimes V_{q}^{\sigma}(\Lambda)$ spanned by $G\left(b_{\mu}\right) \otimes G(b)$ with $b \in B_{k}, k \leq j$, and set

$$
y_{j}=G\left(b_{\mu}\right) \otimes G\left(b_{j}^{\prime}\right) .
$$

Let also $Z_{j}=\sum_{k \leq j} U_{\mathbb{Z}_{q}}\left(\hat{\mathfrak{n}}^{-}\right)\left(G\left(b_{\mu}\right) \otimes G\left(b_{k}\right)\right)$. Since $J$ is linearly ordered and finite, say $\# J=n$ and identify it with $\{1, \ldots, n\}$. For convenience, set $Y_{0}=\{0\}$. Observe that $0=Y_{0} \subset Y_{1} \subset \cdots \subset Y_{k}$ is a filtration of the $U_{\mathbb{Z}_{q}}\left(\hat{\mathfrak{b}}^{+}\right)$-module $G\left(b_{\Lambda_{0}}\right) \otimes V_{\mathbb{Z}_{q}}^{\sigma}(\Lambda)$. The following result was proved in [Joseph 2006, Corollary 5.10].

Theorem 4.3.1. Suppose $\mathfrak{g}$ is simply laced and $\mu\left(h_{i}\right) \leq 1$ for all $i \in \hat{I}$. Then:

(a) The $\mathbb{Z}_{q}$-module $Y_{j}$ is $U_{\mathbb{Z}_{q}}\left(\hat{\mathfrak{n}}^{+}\right)$-stable for all $j \in J$.

(b) For all $j \in J, Y_{j} / Y_{j-1}$ is isomorphic to $V_{\mathbb{Z}_{q}}^{\sigma_{j}}\left(v_{j}\right)$. In particular, $Y_{j} / Y_{j-1}$ is a free $\mathbb{Z}_{q}$-module. 
(c) For all $j \in J$, the image of $\left\{G\left(b_{\mu}\right) \otimes G(b): b \in B_{j}\right\}$ in $Y_{j} / Y_{j-1}$ is a $\mathbb{Z}_{q}$-basis of $Y_{j} / Y_{j-1}$.

(d) For each $j \in J, Z_{j}$ is $U_{\mathbb{Z}_{q}}\left(\hat{\mathfrak{g}}^{\prime}\right)$-stable and $Y_{j}=Z_{j} \cap\left(G\left(b_{\mu}\right) \otimes V_{\mathbb{Z}_{q}}^{\sigma}(\Lambda)\right)$.

Remark 4.3.2. The above theorem was proved in [Joseph 2006] for any simplylaced symmetric Kac-Moody Lie algebra. However, as pointed out in [Naoi 2012, Remark 4.15], the proof also holds for $\hat{\mathfrak{s l}}_{2}$.

It follows from Theorem 4.3.1 and the fact that $G\left(b_{\mu}\right)$ is a highest-weight vector of $V_{q}(\Lambda)$ (4.3.1) that

$$
Y_{j}=\sum_{k \leq j} U_{\mathbb{Z}_{q}}\left(\hat{\mathfrak{n}}^{+}\right) y_{j}
$$

4.4. Simply laced Demazure flags. Given $\ell \geq 0, \lambda \in P^{+}, m \in \mathbb{Z}$, let $D_{\mathbb{F}}(\ell, \lambda, m)=$ $\tau_{m}\left(D_{\mathbb{E}}(\ell, \lambda)\right)$ and $D_{\mathbb{Z}}(\ell, \lambda, m)=\tau_{m}\left(D_{\mathbb{Z}}(\ell, \lambda)\right)$.

Theorem 4.4.1. Suppose $\mathfrak{g}$ is simply laced, let $\mu$ be in $P^{+}$and $\ell^{\prime}>\ell \geq 0$. Then there exist $k>0, \mu_{1}, \ldots, \mu_{k} \in P^{+}, m_{1}, \ldots, m_{k} \in \mathbb{Z}_{\geq 0}$, and a filtration of $U_{\mathbb{Z}}(\mathfrak{g}[t])$ modules $0=D_{0} \subseteq D_{1} \subseteq \cdots \subseteq D_{k}=D_{\mathbb{Z}}(\ell, \mu)$ such that $D_{j}$ and $D_{j} / D_{j-1}$ are free $\mathbb{Z}$-modules for all $j=1, \ldots, k$, and $D_{j} / D_{j-1} \cong D_{\mathbb{Z}}\left(\ell^{\prime}, \mu_{j}, m_{j}\right)$. Moreover, for all $j \in J$, there exists $\vartheta_{j} \in D_{j}$ such that

(i) the image of $\vartheta_{j}$ in $D_{j} / D_{j-1}$ satisfies the defining relations of $D_{\mathbb{Z}}\left(\ell^{\prime}, \mu_{j}, m_{j}\right)$;

(ii) $D_{j}=\sum_{k \leq j} U_{\mathbb{Z}}\left(\mathfrak{n}^{-}[t]\right) \vartheta_{k}$.

Proof. The proof follows closely that of [Naoi 2012, Corollary 4.16]. First notice that it is enough to prove the theorem for $\ell^{\prime}=\ell+1$. Then let $\Lambda \in \widehat{P}^{+}$and $w \in \widehat{\mathscr{W}}$ be such that $w \Lambda=\ell \Lambda_{0}+w_{0} \mu$, and let $V_{\mathbb{Z}_{q}}^{w}(\Lambda)=U_{\mathbb{Z}_{q}}\left(\hat{\mathfrak{n}}^{+}\right) G(b)$ where $b \in B(\Lambda)_{w \Lambda}$.

From Section 4.3, we know that the $U_{\mathbb{Z}_{q}}\left(\hat{\mathfrak{b}}^{+}\right)$-submodule $G\left(b_{\Lambda_{0}}\right) \otimes V_{\mathbb{Z}_{q}}^{w}(\Lambda) \subseteq$ $V_{q}\left(\Lambda_{0}\right) \otimes V_{q}(\Lambda)$ admits a filtration $0=Y_{0} \subset Y_{1} \subset \cdots \subset Y_{k}$. For each $j=1, \ldots, k$, let $D_{j}=\mathbb{Z} \otimes_{\mathbb{Z}_{q}} Y_{j}$, and observe that

$$
\begin{aligned}
D_{k}=\mathbb{Z} \otimes_{\mathbb{Z}_{q}}\left(G\left(b_{\Lambda_{0}}\right)\right. & \left.\otimes_{\mathbb{Z}_{q}} V_{\mathbb{Z}_{q}}^{w}(\Lambda)\right) \\
& \cong\left(\mathbb{Z} \otimes_{\mathbb{Z}_{q}} G\left(b_{\Lambda_{0}}\right)\right) \otimes_{\mathbb{Z}}\left(\mathbb{Z} \otimes_{\mathbb{Z}_{q}} V_{\mathbb{Z}_{q}}^{w}(\Lambda)\right) \cong \mathbb{Z}_{\Lambda_{0}} \otimes_{\mathbb{Z}} D_{\mathbb{Z}}(\ell, \mu),
\end{aligned}
$$

where $\mathbb{Z}_{\Lambda_{0}}$ is a $U_{\mathbb{Z}}\left(\hat{\mathfrak{b}}^{+}\right)$-module on which $U_{\mathbb{Z}}\left(\hat{\mathfrak{n}}^{+}\right)^{0}$ and $U_{\mathbb{Z}}(\mathfrak{g})^{0}$ act trivially and $U_{\mathbb{Z}}(\hat{\mathfrak{h}})$ acts by $\Lambda_{0}$. Moreover, as a $\mathbb{Z}$-module it is free of rank 1 . Thus $D_{k}$ is isomorphic to $D_{\mathbb{Z}}(\ell, \mu)$ as a $U_{\mathbb{Z}}(\mathfrak{g}[t])$-module. It follows from Theorem 4.3.1(d) that $D_{j}$ is a $U_{\mathbb{Z}}(\mathfrak{g}[t])$-module for all $j=1, \ldots, k$ and, hence, so is $D_{j} / D_{j-1}$. So we have a filtration of $U_{\mathbb{Z}}(\mathfrak{g}[t])$-modules $0=D_{0} \subset D_{1} \subset \cdots \subset D_{k}=D_{\mathbb{Z}}(\ell, \mu)$.

By Theorem 4.3.1(b), $Y_{j} / Y_{j-1} \cong V_{\mathbb{Z}_{q}}^{\sigma_{j}}\left(v_{j}\right)$ for some $\sigma_{j} \in \widehat{\mathscr{W}}$ and $v_{j} \in \widehat{P}^{+}$. By (4.1.1) $D_{j} / D_{j-1} \cong V_{\mathbb{Z}}^{\sigma_{j}}\left(v_{j}\right)$. Thus $D_{j} / D_{j-1}$ is isomorphic to $D_{\mathbb{Z}}\left(\ell_{j}, \mu_{j}, m_{j}\right)$ for some $\mu_{j} \in P^{+}, m_{j} \in \mathbb{Z}$ and $\ell_{j}=v_{j}(c)$; see (3.5.3). Since all the weights 
of $V_{q}\left(\Lambda_{0}\right) \otimes V_{q}(\Lambda)$ are of the form $\Lambda+\Lambda_{0}-\eta$ for some $\eta \in \hat{Q}^{+}$, and $\alpha_{i}(c)=0$ for all $i \in \hat{I}$, it follows that $\ell_{j}=\ell+1$ for all $j$.

Keep denoting the image of $y_{j}$ in $D_{j}$ by $y_{j}$ (see (4.3.2)). It follows that $D_{j}=$ $\sum_{k \leq j} U_{\mathbb{Z}}\left(\hat{\mathfrak{n}}^{+}\right) y_{j}$ by (4.3.3). As in Remark 3.5.7, we now replace the "lowest-weight" generators $y_{j}$ by "highest-weight generators". Thus, let $b_{j}^{\prime \prime}$ be the unique element of $B_{j}$ such that $\operatorname{wt}\left(b_{j}^{\prime \prime}\right)=w_{0} \sigma_{j} v_{j}=(\ell+1) \Lambda_{0}+\mu_{j}+m_{j} \delta$ and let $\vartheta_{j}$ be defined similarly to $y_{j}$ by replacing $b_{j}^{\prime}$ by $b_{j}^{\prime \prime}$.

The next corollary is now immediate.

Corollary 4.4.2. Let $\mathfrak{g}, \mu, \ell^{\prime}, \ell, k, \mu_{j}, j=1, \ldots, k$, be as in Theorem 4.4.1. Then there exists a filtration of $U_{\mathbb{F}}(\mathfrak{g}[t])$-modules $0=D_{0} \subseteq D_{1} \subseteq \cdots \subseteq D_{k}=D_{\mathbb{E}}(\ell, \mu)$, such that $D_{j} / D_{j-1} \cong D_{\mathbb{F}}\left(\ell^{\prime}, \mu_{j}\right)$ for all $j=1, \ldots, k$.

\section{Proof of Theorem 1.5.2}

5.1. The isomorphism between Demazure and graded local Weyl modules. Recall that for $\mathfrak{g}=\mathfrak{s l}_{2}$, a characteristic-free proof of Theorem 1.5.2(a) was given in [Jakelić and Moura 2014]. Thus, assume $\mathfrak{g}$ is simply laced of rank higher than 1 and recall from Remark 1.5.1 that $D_{\mathbb{E}}(1, \lambda)$ is a quotient of $W_{\mathbb{F}}^{c}(\lambda)$. To prove the converse, let $w$ be the image of 1 in $W_{\mathbb{F}}^{c}(\lambda)$. In order to show that $W_{\mathbb{F}}^{c}(\lambda)$ is a quotient of $D_{\mathbb{E}}(1, \lambda)$, it remains to prove that

$$
\left(x_{\alpha, s}^{-}\right)^{(k)} w=0 \text { for all } \alpha \in R^{+}, s>0, k>\max \left\{0, \lambda\left(h_{\alpha}\right)-s\right\} .
$$

Given $\alpha \in R^{+}$, consider the subalgebra $U_{\mathbb{F}}\left(\mathfrak{s l}_{\alpha}[t]\right)$ (see Section 2.3) and let $W_{\alpha}$ be the $U_{\mathbb{F}}\left(\mathfrak{s l}_{\alpha}[t]\right)$-submodule of $W_{\mathbb{F}}^{c}(\lambda)$ generated by $w$. Clearly, $W_{\alpha}$ is a quotient of the graded local Weyl module for $U_{\mathbb{E}}\left(\mathfrak{s l}_{\alpha}[t]\right)$ with highest weight $\lambda\left(h_{\alpha}\right)$, where we have identified the weight lattice of $\mathfrak{s l}_{2}$ with $\mathbb{Z}$ as usual. Since we already know that the theorem holds for $\mathfrak{s l}_{2}$, it follows that $w$ must satisfy the same relations as the generator of the corresponding Demazure module for $U_{\mathbb{F}}\left(\mathfrak{s l}_{\alpha}[t]\right)$. In particular, (5.1.1) holds and so does Theorem 1.5.2(a).

5.2. A smaller set of relations for nonsimply laced Demazure modules. In this subsection we assume $\mathfrak{g}$ is not simply laced and prove the following analogue of [Naoi 2012, Proposition 4.1].

Proposition 5.2.1. For all $\lambda \in P^{+}, D_{\mathbb{F}}(1, \lambda)$ is isomorphic to the quotient of $U_{\mathbb{F}}(\mathfrak{g}[t])$ by the left ideal $I_{\mathbb{F}}(\lambda)$ generated by

$$
U_{\mathbb{F}}\left(\mathfrak{n}^{+}[t]\right)^{0}, \quad U_{\mathbb{F}}\left(\mathfrak{h}[t]_{+}\right)^{0}, \quad h-\lambda(h), \quad\left(x_{i}^{-}\right)^{(k)}, \quad\left(x_{\alpha, s}^{-}\right)^{(\ell)}
$$

for all $h \in U_{\mathbb{F}}(\mathfrak{h}), i \in I \backslash I_{\mathrm{sh}}, \alpha \in R_{\mathrm{sh}}^{+}, s \geq 0, k>\lambda\left(h_{i}\right), \ell>\max \left\{0, \lambda\left(h_{\alpha}\right)-s r^{\vee}\right\}$.

Let $w$ be in $D_{\mathbb{F}}(1, \lambda)_{\lambda} \backslash\{0\}$ and $V$ be the $U_{\mathbb{F}}(\mathfrak{g}[t])$-module generated by a vector $v$ with defining relations given by (5.2.1). In particular, there exists a unique 
epimorphism $V \rightarrow D_{\mathbb{F}}(1, \lambda)$ mapping $v$ to $w$. To prove the converse, observe first that since $\left(x_{i}^{-}\right)^{(k)} v=0$ for all $i \in I, k>\lambda\left(h_{i}\right)$, Lemma 3.1.5 implies that $\left(x_{\alpha}^{-}\right)^{(k)} v=0$ for all $\alpha \in R^{+}, k>\lambda\left(h_{\alpha}\right)$. In particular, $V$ is a quotient of $W_{\mathbb{F}}^{c}(\lambda)$ and, hence, it is finite-dimensional. It remains to show that

$$
\left(x_{\alpha, s}^{-}\right)^{(k)} v=0 \text { for all } \alpha \in R^{+} \backslash R_{\mathrm{sh}}^{+}, s>0, k>\max \left\{0, \lambda\left(h_{\alpha}\right)-s r_{\alpha}^{\vee}\right\} .
$$

These relations will follow from the next few lemmas.

Lemma 5.2.2. Let $V$ be a finite-dimensional $U_{\mathbb{F}}(\mathfrak{g}[t])$-module, $\lambda$ be in $P^{+}$, and suppose $v \in V_{\lambda}$ satisfies $U_{\mathbb{F}}\left(\mathfrak{n}^{+}[t]\right)^{0} v=U_{\mathbb{F}}\left(\mathfrak{h}[t]_{+}\right)^{0} v=0$. If $\alpha \in R^{+}$is long, then $\left(x_{\alpha, s}^{-}\right)^{(k)} v=0$ for all $s \geq 0, k>\max \left\{0, \lambda\left(h_{\alpha}\right)-s\right\}$.

Proof. Consider the subalgebra $U_{\mathbb{F}}\left(\mathfrak{s l}_{\alpha}[t]\right)$ (see Section 2.3). By Theorem 3.3.4 (c), the submodule $W=U_{\mathbb{F}}\left(\mathfrak{s l}_{\alpha}[t]\right) v$ is a quotient of the local graded Weyl module for $U_{\mathbb{F}}\left(\mathfrak{s l}_{\alpha}[t]\right)$ with highest weight $\lambda\left(h_{\alpha}\right)$. Theorem 1.5.2 (a) implies that $W \cong$ $D_{\mathbb{F}}^{\alpha}\left(1, \lambda\left(h_{\alpha}\right)\right)$ where the latter is the corresponding Demazure module for $U_{\mathbb{F}}\left(\mathfrak{s l}_{\alpha}[t]\right)$. In particular, $v$ satisfies the relations (1.5.2).

Lemma 5.2.3. Assume $\mathfrak{g}$ is not of type $G_{2}$. Let $V$ be a finite-dimensional $U_{\mathbb{F}}(\mathfrak{g}[t])$ module, $\lambda$ be in $P^{+}$, and suppose $v \in V_{\lambda}$ satisfies $U_{\mathbb{F}}\left(\mathfrak{n}^{+}[t]\right)^{0} v=U_{\mathbb{F}}\left(\mathfrak{h}[t]_{+}\right)^{0} v=0$ and $\left(x_{\alpha, s}^{-}\right)^{(k)} v=0$ for all $\alpha \in R_{\mathrm{sh}}^{+}, k>\max \left\{0, \lambda\left(h_{\alpha}\right)-2 s\right\}$. Then for every short root $\gamma$, we have $\left(x_{\gamma, s}^{-}\right)^{(k)} v=0$ for all $s \geq 0, k>\max \left\{0, \lambda\left(h_{\gamma}\right)-2 s\right\}$.

Proof. The proof will proceed by induction on $\mathrm{ht}(\gamma)$. If $\mathrm{ht}(\gamma)=1$, then $\gamma$ is simple and, hence, $\gamma \in R_{\mathrm{sh}}^{+}$. Thus, suppose ht $(\gamma)>1$ and that $\gamma \notin R_{\mathrm{sh}}^{+}$. By [Naoi 2012, Lemma 4.6], there exist $\alpha, \beta \in R^{+}$such that $\gamma=\alpha+\beta$ with $\alpha$ long and $\beta$ short. Notice that $\{\alpha, \beta\}$ form a simple system of a rank-two root subsystem. In particular, $h_{\gamma}=2 h_{\alpha}+h_{\beta}$ and, hence, $\lambda\left(h_{\gamma}\right)=2 \lambda\left(h_{\alpha}\right)+\lambda\left(h_{\beta}\right)$.

Fix $s \geq 0$ and suppose first that $\lambda\left(h_{\gamma}\right)-2 s \geq 0$. In this case, we can choose $a, b \in \mathbb{Z}_{\geq 0}$ such that

$$
a+b=s, \quad \lambda\left(h_{\alpha}\right)-a \geq 0, \quad \text { and } \quad \lambda\left(h_{\beta}\right)-2 b \geq 0 .
$$

Indeed, $b=\max \left\{0, s-\lambda\left(h_{\alpha}\right)\right\}$ and $a=s-b$ satisfy these conditions. Then Lemma 5.2.2 implies that $\left(x_{\alpha, a}^{-}\right)^{(k)} v=0$ for all $k>\lambda\left(h_{\alpha}\right)-a$, while the induction hypothesis implies that $\left(x_{\beta, b}^{-}\right)^{(k)} v=0$ for all $k>\lambda\left(h_{\beta}\right)-2 b$. Applying Lemma 3.1.5 to the subalgebra $U_{\mathbb{F}}\left(\mathfrak{g}_{\alpha, \beta}^{a, b}\right)$ (see Section 2.3), it follows that $\left(x_{\gamma, s}^{-}\right)^{(k)} v=0$ for all $k>2\left(\lambda\left(h_{\alpha}\right)-a\right)+\left(\lambda\left(h_{\beta}\right)-2 b\right)=\lambda\left(h_{\gamma}\right)-2 s$.

Now suppose $\lambda\left(h_{\gamma}\right)-2 s \leq 0$; this implies $s-\lambda\left(h_{\alpha}\right)=s-\frac{1}{2}\left(\lambda\left(h_{\gamma}\right)-\lambda\left(h_{\beta}\right)\right) \geq$ $\lambda\left(h_{\beta}\right) / 2 \geq 0$. We need to show that $\left(x_{\gamma, s}^{-}\right)^{(k)} v=0$ for all $k>0$. Letting $a=\lambda\left(h_{\alpha}\right)$ and $b=s-\lambda\left(h_{\alpha}\right)$, we have

$$
a+b=s, \quad \lambda\left(h_{\alpha}\right)-a \leq 0, \quad \text { and } \quad \lambda\left(h_{\beta}\right)-2 b \leq 0 .
$$


Then Lemma 5.2.2 implies that $\left(x_{\alpha, a}^{-}\right)^{(k)} v=0$ for all $k>0$, while the induction hypothesis implies that $\left(x_{\beta, b}^{-}\right)^{(k)} v=0$ for all $k>0$. The result follows from an application of Lemma 3.1.5 as before.

It remains to prove an analogue of Lemma 5.2.3 for $\mathfrak{g}$ of type $G_{2}$. This is much more technically complicated and will require that we assume that characteristic of $\mathbb{F}$ is at least 5. For the remainder of this subsection we assume $\mathfrak{g}$ is of type $G_{2}$ and set $I=\{1,2\}$ so that $\alpha_{1}$ is short. Given $\gamma=s \alpha_{1}+l \alpha_{2} \in R^{+}$, set $s_{\gamma}=s$. Set also

$\mathfrak{n}^{+}[t]_{>}=\bigoplus_{\gamma \in R^{+}} \bigoplus_{s \geq s_{\gamma}} \mathbb{C} x_{\gamma, s}^{+}, \quad \mathfrak{n}^{+}[t]_{<}=\bigoplus_{\gamma \in R^{+}} \bigoplus_{s=0}^{s_{\gamma}-1} \mathbb{C} x_{\gamma, s}^{+}, \quad \mathfrak{a}=\mathfrak{n}^{-}[t] \oplus \mathfrak{h}[t] \oplus \mathfrak{n}^{+}[t]_{>}$,

and observe that $\mathfrak{n}^{+}[t]_{>}$and $\mathfrak{n}^{+}[t]_{<}$are subalgebras of $\mathfrak{n}^{+}[t]$ such that $\mathfrak{n}^{+}[t]=$ $\mathfrak{n}^{+}[t]_{>} \oplus \mathfrak{n}^{+}[t]_{<}$. The hyperalgebras $U_{\mathbb{F}}\left(\mathfrak{n}^{+}[t]_{>}\right), U_{\mathbb{F}}\left(\mathfrak{n}^{+}[t]_{<}\right)$, and $U_{\mathbb{F}}(\mathfrak{a})$ are then defined in the usual way (see Section 1.3) and the PBW theorem implies that

$$
U_{\mathbb{F}}\left(\mathfrak{n}^{+}[t]\right)=U_{\mathbb{F}}\left(\mathfrak{n}^{+}[t]_{>}\right) \oplus U_{\mathbb{F}}\left(\mathfrak{n}^{+}[t]\right) U_{\mathbb{F}}\left(\mathfrak{n}^{+}[t]_{<}\right)^{0} .
$$

We now prove a version of [Naoi 2012, Lemma 4.11] for hyperalgebras.

Lemma 5.2.4. Given $\lambda \in P^{+}$, let $I_{\mathbb{F}}^{\prime}(\lambda)$ be the left ideal of $U_{\mathbb{F}}(\mathfrak{a})$ generated by the generators of $I_{\mathbb{F}}(\lambda)$ described in (5.2.1) which lie in $U_{\mathbb{F}}(\mathfrak{a})$. Then

$$
I_{\mathbb{F}}(\lambda) \subseteq I_{\mathbb{F}}^{\prime}(\lambda) \oplus U_{\mathbb{F}}(\mathfrak{a}) U_{\mathbb{F}}\left(\mathfrak{n}^{+}[t]_{<}\right)^{0} .
$$

Proof. Recall that $I_{\mathbb{F}}(\lambda)$ is the left ideal of $U_{\mathbb{F}}(\mathfrak{g}[t])$ generated by the set $\mathscr{I}$ whose elements are the elements in $U_{\mathbb{F}}\left(\mathfrak{n}^{+}[t]\right)^{0}, U_{\mathbb{F}}\left(\mathfrak{h}[t]_{+}\right)^{0}$, together with the elements

$$
\left(\begin{array}{c}
h_{i} \\
l
\end{array}\right)-\left(\begin{array}{c}
\lambda\left(h_{i}\right) \\
l
\end{array}\right), \quad\left(x_{2}^{-}\right)^{(m)}, \quad\left(x_{1, s}^{-}\right)^{(k)}
$$

for $i \in I, k, l, m, s \in \mathbb{Z}_{\geq 0}, m>\lambda\left(h_{2}\right), k>\max \left\{0, \lambda\left(h_{1}\right)-3 s\right\}$. To simplify notation, set $U_{<}=U_{\mathbb{F}}\left(\mathfrak{n}^{+}[t]_{<}\right)$and $J=I_{\mathbb{F}}^{\prime}(\lambda) \oplus U_{\mathbb{F}}(\mathfrak{a}) U_{\mathbb{F}}\left(\mathfrak{n}^{+}[t]_{<}\right)^{0}$. Observe that $U_{\mathbb{F}}(\mathfrak{a}) J \subseteq J$. Therefore, since $U_{\mathbb{F}}(\mathfrak{g}[t])=U_{\mathbb{F}}(\mathfrak{a}) U_{<}$by (5.2.2) and we clearly have $\mathscr{I} \subseteq J$, it suffices to show that

$$
U_{<}^{0} \mathscr{I} \subseteq J
$$

We will decompose the set $\mathscr{I}$ into parts, and prove the inclusion for each part. Namely, we first decompose $\mathscr{I}$ into $\left(\mathscr{I} \cap U_{\mathbb{F}}\left(\mathfrak{n}^{+}[t]\right) U_{\mathbb{F}}(\mathfrak{h}[t])\right) \sqcup\left(\mathscr{I} \cap U_{\mathbb{F}}\left(\mathfrak{n}^{-}[t]\right)\right)$, and then we further decompose $\mathscr{I} \cap U_{\mathbb{F}}\left(\mathfrak{n}^{-}[t]\right)$ as

$$
\left\{\left(x_{2}^{-}\right)^{(m)}: m>\lambda\left(h_{2}\right)\right\} \sqcup\left\{\left(x_{1, s}^{-}\right)^{(k)}: s \in \mathbb{Z}_{\geq 0}, k>\max \left\{0, \lambda\left(h_{1}\right)-3 s\right\}\right\} .
$$

Since $\mathfrak{h}[t] \oplus \mathfrak{n}^{+}[t]$ is a subalgebra of $\mathfrak{g}[t]$, the PBW theorem tells us that $U_{\mathbb{F}}\left(\mathfrak{n}^{+}[t]\right) U_{\mathbb{F}}(\mathfrak{h}[t])=U_{\mathbb{F}}(\mathfrak{h}[t]) U_{\mathbb{F}}\left(\mathfrak{n}^{+}[t]\right)$, and therefore

$$
U_{<}^{0}\left(\mathscr{I} \cap U_{\mathbb{F}}\left(\mathfrak{n}^{+}[t]\right) U_{\mathbb{F}}(\mathfrak{h}[t])\right) \subseteq U_{\mathbb{F}}(\mathfrak{h}[t]) U_{\mathbb{F}}\left(\mathfrak{n}^{+}[t]\right) .
$$


Now, by (5.2.2), $U_{\mathbb{F}}(\mathfrak{h}[t]) U_{\mathbb{F}}\left(\mathfrak{n}^{+}[t]\right) \subseteq J$, so $U_{<}^{0}\left(\mathscr{\Phi} \cap U_{\mathbb{F}}\left(\mathfrak{n}^{+}[t]\right) U_{\mathbb{F}}(\mathfrak{h}[t])\right) \subseteq J$. In particular, we have shown that

$$
U_{\mathbb{F}}(\mathfrak{g}[t]) U_{\mathbb{F}}\left(\mathfrak{n}^{+}[t]\right)^{0} \subseteq J .
$$

It remains to show that

$$
U_{<}^{0}\left(\mathscr{I} \cap U_{\mathbb{F}}\left(\mathfrak{n}^{-}[t]\right)\right) \subseteq J .
$$

We begin by proving that $U_{<}^{0} U_{\mathbb{F}}\left(\mathfrak{n}_{2}^{-}\right) \subseteq J$, where $\mathfrak{n}_{2}^{-}$is the subalgebra spanned by $x_{2}^{-}$. Consider the natural $Q$-grading on $U_{\mathbb{F}}(\mathfrak{g}[t])$, and for $\eta \in Q$ let $U_{\mathbb{F}}(\mathfrak{g}[t])_{\eta}$ denote the corresponding graded piece. Observe that $\mathfrak{m}_{2}:=\mathfrak{n}^{+}[t]_{<} \oplus \mathfrak{n}_{2}^{-}$is a subalgebra of $\mathfrak{g}[t]$ and that

$$
U_{<}^{0} U_{\mathbb{F}}\left(\mathfrak{n}_{2}^{-}\right) \subseteq \bigoplus_{\eta} U_{\mathbb{F}}\left(\mathfrak{m}_{2}\right)_{\eta},
$$

where the sum runs over $\mathbb{Z}_{>0} \alpha_{1} \oplus \mathbb{Z} \alpha_{2}$. Together with the PBW theorem, this implies that

$$
U_{<}^{0} U_{\mathbb{F}}\left(\mathfrak{n}_{2}^{-}\right) \subseteq U_{\mathbb{F}}\left(\mathfrak{n}_{2}^{-}\right) U_{<}^{0} \subseteq U_{\mathbb{F}}(\mathfrak{a}) U_{<}^{0} \subseteq J .
$$

Finally, we show that $U_{<}^{0} \mathscr{I}_{1} \subseteq J$, where $\mathscr{I}_{1}=\left(\mathscr{I} \cap U_{\mathbb{F}}\left(\mathfrak{n}_{1}^{-}[t]\right)\right)$ and $\mathfrak{n}_{1}^{-}$is the subalgebra spanned by $x_{1}^{-}$. Consider

$$
\mathfrak{n}^{+}[t]_{<}^{1}=\bigoplus_{\gamma \in R^{+} \backslash\left\{\alpha_{1}\right\}} \bigoplus_{s=0}^{s_{\gamma}-1} \mathbb{C} x_{\gamma, s}^{+},
$$

which is a subalgebra of $\mathfrak{n}^{+}[t]_{<}$such that $\mathfrak{n}^{+}[t]_{<}=\mathfrak{n}_{1}^{+} \oplus \mathfrak{n}^{+}[t]_{<}^{1}$, where $\mathfrak{n}_{1}^{+}=\mathbb{C} x_{1}^{+}$. Moreover, $\mathfrak{m}_{1}:=\mathfrak{n}^{+}[t]_{<}^{1} \oplus \mathfrak{n}_{1}^{-}[t]$ is a subalgebra of $\mathfrak{g}[t]$ such that $U\left(\mathfrak{m}_{1}\right)_{\eta} \neq 0$ only if $\eta \in \mathbb{Z} \alpha_{1} \oplus \mathbb{Z}_{\geq 0} \alpha_{2}$ and $U\left(\mathfrak{m}_{1}\right)_{0}=\mathbb{C}$. This implies that

$$
U_{\mathbb{F}}\left(\mathfrak{n}^{+}[t]_{<}^{1}\right)^{0} U_{\mathbb{F}}\left(\mathfrak{n}_{1}^{-}[t]\right)=U_{\mathbb{F}}\left(\mathfrak{n}_{1}^{-}[t]\right) U_{\mathbb{F}}\left(\mathfrak{n}^{+}[t]_{<}^{1}\right)^{0} .
$$

Since $U_{<}^{0}=U_{\mathbb{F}}\left(\mathfrak{n}_{1}^{+}\right) U_{\mathbb{F}}\left(\mathfrak{n}^{+}[t]_{<}^{1}\right)^{0} \oplus U_{\mathbb{F}}\left(\mathfrak{n}_{1}^{+}\right)^{0}$, we get

$$
\begin{aligned}
U_{<}^{0} \Phi_{1} & \subseteq\left(U_{\mathbb{F}}\left(\mathfrak{n}_{1}^{+}\right) U_{\mathbb{F}}\left(\mathfrak{n}^{+}[t]_{<}^{1}\right)^{0}+U_{\mathbb{F}}\left(\mathfrak{n}_{1}^{+}\right)^{0}\right) \mathscr{I}_{1} \\
& \subseteq U_{\mathbb{F}}\left(\mathfrak{n}_{1}^{+}\right) U_{\mathbb{F}}\left(\mathfrak{n}_{1}^{-}[t]\right) U_{\mathbb{F}}\left(\mathfrak{n}^{+}[t]_{<}^{1}\right)^{0}+U_{\mathbb{F}}\left(\mathfrak{n}_{1}^{+}\right)^{0} \Phi_{1} \\
& \subseteq U_{\mathbb{F}}(\mathfrak{g}[t]) U_{\mathbb{F}}\left(\mathfrak{n}^{+}[t]\right)^{0}+U_{\mathbb{F}}\left(\mathfrak{n}_{1}^{+}\right)^{0} \mathscr{I}_{1} .
\end{aligned}
$$

The first summand in the last line is in $J$ by (5.2.3) while the second one is in $J$ by Corollary 3.5.8 (with $\lambda=\lambda\left(h_{1}\right)$ and $\ell=3$ ) together with (5.2.3).

Set $\mathfrak{h}_{i}=\mathbb{C} h_{i}, i \in I$, and $\mathfrak{b}=\mathfrak{n}^{-}[t] \oplus \mathfrak{h}[t]_{+} \oplus \mathfrak{h}_{2} \oplus \mathfrak{n}^{+}[t]_{>}$. Observe that $\mathfrak{b}$ is an ideal of $\mathfrak{a}$ such that $\mathfrak{a}=\mathfrak{b} \oplus \mathfrak{h}_{1}$. One easily checks that there exists a unique Lie algebra homomorphism $\phi: \mathfrak{b} \rightarrow \mathfrak{g}[t]$ such that

$$
\phi\left(x_{\gamma, r}^{ \pm}\right)=x_{\gamma, r \mp s_{\gamma}}^{ \pm} \text {for all } \gamma \in R^{+} .
$$


Moreover, $\phi$ is the identity on $\mathfrak{h}[t]_{+}+\mathfrak{s l}_{\alpha_{2}}$. Also, $\phi$ can be extended to a Lie algebra map $\mathfrak{a} \rightarrow U\left(\mathfrak{g}[t]\right.$ ) by setting $\phi\left(h_{1}\right)=h_{1}-3$ (see [Naoi 2012, Section 4.2]). Proceeding as in Section 2.2, one sees that $\phi$ induces an algebra homomorphism $U_{\mathbb{F}}(\mathfrak{a}) \rightarrow U_{\mathbb{F}}(\mathfrak{g}[t])$ also denoted by $\phi$.

We are ready to prove the analogue of Lemma 5.2.3 for type $G_{2}$.

Lemma 5.2.5. Let $V$ be a finite-dimensional $U_{\mathbb{F}}(\mathfrak{g}[t])$-module, $\lambda \in P^{+}$, and suppose $v \in V_{\lambda}$ satisfies $U_{\mathbb{F}}\left(\mathfrak{n}^{+}[t]\right)^{0} v=U_{\mathbb{F}}\left(\mathfrak{h}[t]_{+}\right)^{0} v=0$ and $\left(x_{1, s}^{-}\right)^{(k)} v=0$ for all $k>$ $\max \left\{0, \lambda\left(h_{1}\right)-3 s\right\}$. Then for every short root $\gamma$, we have $\left(x_{\gamma, s}^{-}\right)^{(k)} v=0$ for all $s \geq 0, k>\max \left\{0, \lambda\left(h_{\gamma}\right)-3 s\right\}$.

Proof. Notice that the conclusion of the lemma is equivalent to

$$
\left(x_{\gamma, s}^{-}\right)^{(k)} \in I_{\mathbb{F}}(\lambda) \text { for all } s \geq 0, k>\max \left\{0, \lambda\left(h_{\gamma}\right)-3 s\right\}
$$

for every short root $\gamma$. Recall that the short roots in $R^{+}$are $\alpha_{1}, \alpha:=\alpha_{1}+\alpha_{2}$ and $\vartheta:=2 \alpha_{1}+\alpha_{2}$ while the long roots are $\alpha_{2}, \beta:=3 \alpha_{1}+\alpha_{2}$ and $\theta:=3 \alpha_{1}+2 \alpha_{2}$. For $\gamma=\alpha$, we have $h_{\gamma}=h_{1}+3 h_{2}$ and the proof is similar to that of Lemma 5.2.3 (the details can be found in [Macedo 2013]). We shall use that the lemma holds for $\gamma=\alpha$ in the remainder of the proof. It remains to show that the lemma holds with $\gamma=\vartheta$. Notice that $h_{\vartheta}=2 h_{1}+3 h_{2}$ and thus we want to prove that

$$
\left(x_{\vartheta, s}^{-}\right)^{(k)} \in I_{\mathbb{F}}(\lambda) \text { for all } s \geq 0, k>\max \left\{0,2 \lambda\left(h_{1}\right)+3 \lambda\left(h_{2}\right)-3 s\right\} .
$$

We prove (5.2.4) by induction on $\lambda\left(h_{1}\right)$. Following [Naoi 2012], we prove the cases $\lambda\left(h_{1}\right) \in\{0,1,2\}$ and then we show that (5.2.4) for $\lambda-3 \omega_{1}$ in place of $\lambda$ implies it for $\lambda$. To shorten notation, set $a=\lambda\left(h_{1}\right), b=\lambda\left(h_{2}\right)$.

(1) Assume $a=0$. Since $\alpha_{1} \in R_{\mathrm{sh}}^{+}$, it follows that $\left(x_{1}^{-}\right)^{(k)} v=0$ for all $k>0$. By Lemma 5.2.2, we have $\left(x_{2, s}^{-}\right)^{(k)} v=0$ for all $k>\max \{0, b-s\}$. Applying Lemma 3.1.5 to the subalgebra $U_{\mathbb{F}}\left(\mathfrak{g}_{\alpha_{1}, \alpha_{2}}^{0, s}\right)$, it follows that $\left(x_{\vartheta, s}^{-}\right)^{(k)} v=0$ for all $k>3 \max \{0, b-s\}=\max \{0,2 a+3 b-3 s\}$ as desired.

(2) Assume $a=1$. This time we have $\left(x_{1}^{-}\right)^{(k)} v=0$ for all $k>1$. We split in 3 subcases.

(2.1) Suppose $b>s-1$, and notice $2 a+3 b-3 s>0$. Lemma 5.2.2 implies $\left(x_{2, s}^{-}\right)^{(k)} v=0$ for all $k>\max \{0, b-s\}=b-s$. Applying Lemma 3.1.5 to the subalgebra $U_{\mathbb{F}}\left(\mathfrak{g}_{\alpha_{1}, \alpha_{2}}^{0, s}\right)$, it follows that $\left(x_{\vartheta, s}^{-}\right)^{(k)} v=0$ for all $k>2+3(b-s)=$ $2 a+3 b-3 s$.

(2.2) Suppose $b=s-1$, in which case $2 a+3 b-3 s<0$. Notice that $h_{\beta}=h_{1}+h_{2}$ and, hence, $\lambda\left(h_{\beta}\right)=a+b=s$. Lemma 5.2.2 then implies that $\left(x_{\beta, s}^{-}\right)^{(k)} v=0$ for all $k>0$. Notice that $\left\{-\alpha_{1}, \beta\right\}$ form a basis for $R$. Since, $\left(x_{1}^{+}\right)^{(k)} v=0$ for all $k>0$, Lemma 3.1.5 applied to the subalgebra $U_{\mathbb{F}}\left(\mathfrak{g}_{-\alpha_{1}, \beta}^{0, s}\right)$ implies that $\left(x_{\vartheta, s}^{-}\right)^{(k)} v=0$ for all $k>0$. 
(2.3) Suppose $b<s-1$, in which case $2 a+3 b-3 s<0$. This time we apply Lemma 3.1 .5 to the subalgebra $U_{\mathbb{F}}\left(\mathfrak{g}_{\alpha_{1}, \alpha_{2}}^{1, s-2}\right)$. Indeed, we have $\left(x_{1,1}^{-}\right)^{(k)} v=0$ for all $k>\max \{0, a-3\}=0$ and Lemma 5.2.2 implies that $\left(x_{2, s-2}^{-}\right)^{(k)} v=0$ for all $k>\max \{0, b-(s-2)\}=0$. Thus, since $3(b-s)<-3$ and $a=1$, we have $\max \{0,2 a+3 b-3 s\}=0$ and Lemma 3.1 .5 implies that $\left(x_{\vartheta, s}^{-}\right)^{(k)} v=0$ for all $k>0$.

(3) Assume $a=2$. We split in subcases as before.

(3.1) If $b>s-1$, the proof is similar to that of step (2.1).

(3.2) Suppose $b=s-1$, and notice that $2 a+3 b-3 s=1$. Hence, we want to show that (5.2.4) holds for $k>1$. For $k>3$ we apply Lemma 3.1.5 to the subalgebra $U_{\mathbb{F}}\left(\mathfrak{g}_{\alpha_{1}, \alpha_{2}}^{1, s-2}\right)$ in a similar fashion as we did in step (2.3) (the same can be conclude using the argument from step (2.2). For $k \in\{2,3\}$ we need our hypothesis on the characteristic of $\mathbb{F}$. Assume we have chosen the Chevalley basis so that $x_{\vartheta}^{-}=\left[x_{1}^{+}, x_{\beta}^{-}\right]$and observe that (1.1.4) implies that $\left[x_{1}^{+}, x_{\vartheta}^{-}\right]= \pm 2 x_{\alpha}^{-}$. Using this, one easily checks that

$$
\left(x_{\vartheta, s}^{-}\right)^{(2)}=\left(x_{1}^{+}\right)^{(2)}\left(x_{\beta, s}^{-}\right)^{(2)}-\frac{1}{2} x_{1}^{+}\left(x_{\beta, s}^{-}\right)^{(2)} x_{1}^{+}-\frac{1}{2} x_{\beta, s}^{-} x_{\vartheta, s}^{-} x_{1}^{+} \mp x_{\beta, s}^{-} x_{\alpha, s}^{-} .
$$

Using the case $\gamma=\alpha$ and Lemma 5.2.2 we see that $x_{\alpha, s}^{-} v=\left(x_{\beta, s}^{-}\right)^{(2)} v=0$. Hence, since $2 \in \mathbb{F}^{\times},(5.2 .4)$ holds for $k=2$. For $k=3$, we have $\left(x_{\vartheta, s}^{-}\right)^{(3)}=\frac{1}{3} x_{\vartheta, s}^{-}\left(x_{\vartheta, s}^{-}\right)^{(2)}$ and, since $3 \in \mathbb{F}^{\times},(5.2 .4)$ also holds for $k=3$.

(3.3) If $b<s-1$ the proof is similar to that of step (2.3).

(4) Assume $a \geq 3$ and that (5.2.4) holds for $\lambda-3 \omega_{1}$.

(4.1) Suppose $s \geq 2$ and recall the definition of the map $\phi: U_{\mathbb{F}}(\mathfrak{a}) \rightarrow U_{\mathbb{F}}(\mathfrak{g}[t])$. The induction hypothesis together with Lemma 5.2.4 implies that

$$
\left(x_{\vartheta, s-2}^{-}\right)^{(k)} \in I_{\mathbb{F}}^{\prime}\left(\lambda-3 \omega_{1}\right) \text { for all } k>\max \{0,2 a+3 b-3 s\},
$$

and therefore

$$
\left(x_{\vartheta, s}^{-}\right)^{(k)}=\phi\left(\left(x_{\vartheta, s-2}^{-}\right)^{(k)}\right) \in \phi\left(I_{\mathbb{F}}^{\prime}\left(\lambda-3 \omega_{1}\right)\right) \text { for all } k>\max \{0,2 a+3 b-3 s\} .
$$

One easily checks that $\phi$ sends the generators of $I_{\mathbb{F}}^{\prime}\left(\lambda-3 \omega_{1}\right)$ to generators of $I_{\mathbb{F}}(\lambda)$, completing the proof of (5.2.4) for $s \geq 2$.

(4.2) For $s=0$, notice that $U_{\mathbb{F}}(\mathfrak{g}) v$ is a quotient of $W_{\mathbb{F}}(\lambda)$, and (5.2.4) follows. Equivalently, apply Lemma 3.1.5 to $U_{\mathbb{F}}\left(\mathfrak{g}_{\alpha_{1}, \alpha_{2}}^{0,0}\right)=U_{\mathbb{F}}(\mathfrak{g})$ and the proof is similar to that of step (2.1).

(4.3) If $s=1$ and $b \geq 1$, we have $2 a+3 b-3 s>0$ and the usual application of Lemma 3.1.5 to $U_{\mathbb{F}}\left(\mathfrak{g}_{\alpha_{1}, \alpha_{2}}^{0,1}\right)$ completes the proof of (5.2.4). If $s=1$ and $b=0$, we need to show that $\left(x_{\vartheta, 1}^{-}\right)^{(k)} v=0$ for $k>2 a-3$.

Consider the subalgebra $U_{\mathbb{F}}\left(\mathfrak{s l}_{\vartheta}[t]\right) \cong U_{\mathbb{F}}\left(\mathfrak{s l}_{2}[t]\right)$ defined in Section 2.3. Since $\lambda\left(h_{\vartheta}\right)=2 a$, it follows that $W:=U_{\mathbb{F}}\left(\mathfrak{s l}_{\vartheta}[t]\right) v$ is a quotient of the $U_{\mathbb{F}}\left(\mathfrak{s l}_{2}[t]\right)$-module $W_{\mathbb{F}}^{c}(2 a)$, where we identified the weight lattice of $\mathfrak{s l}_{2}$ with $\mathbb{Z}$ as usual. Since 
$W_{\mathbb{F}}^{c}(2 a) \cong D_{\mathbb{F}}(1,2 a)$ by Theorem 1.5.2(a), the defining relations of $D_{\mathbb{F}}(1,2 a)$ imply $\left(x_{\vartheta, 1}^{-}\right)^{(k)} v=0$ for $k>2 a-1$. It remains to check that $\left(x_{\vartheta, 1}^{-}\right)^{(k)} v=0$ for $k \in\{2 a-2,2 a-1\}$.

Suppose by contradiction that $\left(x_{\vartheta, 1}^{-}\right)^{(2 a-1)} v \neq 0$, and notice that

$$
\left(x_{\vartheta}^{-}\right)^{(k)}\left(x_{\vartheta, 1}^{-}\right)^{(2 a-1)} v=0 \text { for all } k>0 .
$$

Indeed,

$$
\left(x_{\vartheta}^{-}\right)^{(k)}\left(x_{\vartheta, 1}^{-}\right)^{(2 a-1)} v \in W_{\mathbb{F}}^{c}(2 a)_{-2 a-2(k-1)}
$$

is a vector of degree $2 a-1>1$ for all $k \geq 0$. By the Weyl group invariance of the character of $W_{\mathbb{F}}^{c}(2 a)$, we know that $W_{\mathbb{F}}^{c}(2 a)_{-2 a-2(k-1)}=0$ if $k>1$, and that $W_{\mathbb{F}}^{c}(2 a)_{-2 a-2(k-1)}$ is one-dimensional concentrated in degree zero if $k=1$. This proves (5.2.5). Then Lemma 3.1.3 implies that

$$
\left(x_{\vartheta}^{+}\right)^{(2 a-2)}\left(x_{\vartheta, 1}^{-}\right)^{(2 a-1)} v \neq 0 .
$$

On the other hand, it follows from Lemma 2.1.1 that

$$
\left(x_{\vartheta}^{+}\right)^{(2 a-2)}\left(x_{\vartheta, 1}^{-}\right)^{(2 a-1)} v=x_{\vartheta, 2 a-1}^{-} v .
$$

Since $2 a-1 \geq 2$ and $2 a-3(2 a-1)=-4 a+3<0$, it follows from step (4.1) that $x_{\vartheta, 2 a-1}^{-} v=0$ yielding a contradiction as desired.

Similarly, assume by contradiction that $\left(x_{\vartheta, 1}^{-}\right)^{(2 a-2)} v \neq 0$ and notice that

$$
\left(x_{\vartheta}^{-}\right)^{(k)}\left(x_{\vartheta, 1}^{-}\right)^{(2 a-2)} v=0 \text { for all } k>1 .
$$

Suppose first that $x_{\vartheta}^{-}\left(x_{\vartheta, 1}^{-}\right)^{(2 a-2)} v=0$ as well. It then follows from Lemma 3.1.3 that

$$
\left(x_{\vartheta}^{+}\right)^{(2 a-4)}\left(x_{\vartheta, 1}^{-}\right)^{(2 a-2)} v \neq 0 .
$$

On the other hand, Lemma 2.1.1 implies that

$$
\left(x_{\vartheta}^{+}\right)^{(2 a-4)}\left(x_{\vartheta, 1}^{-}\right)^{(2 a-2)} v=\left(x_{\vartheta, a-1}^{-}\right)^{(2)} v+\sum_{r=a}^{2 a-2} x_{\vartheta, 2 a-2-r}^{-} x_{\vartheta, r}^{-} v .
$$

Since $a-1 \geq 2$, step (4.1) implies that $\left(x_{\vartheta, r}^{-}\right)^{(k)} v=0$ for all $r \geq a-1, k>0$, implying that the right-hand side is zero, which is a contradiction. It remains to check the possibility that $x_{\vartheta}^{-}\left(x_{\vartheta, 1}^{-}\right)^{(2 a-2)} v \neq 0$. In this case it follows that $x_{\vartheta}^{-}\left(x_{\vartheta, 1}^{-}\right)^{(2 a-2)} v$ is a lowest-weight vector for the algebra $U_{\mathbb{F}}\left(\mathfrak{s l}_{\vartheta}\right)$ and, hence, Lemma 3.1.3 implies that

$$
\left(x_{\vartheta}^{+}\right)^{(2 a-2)} x_{\vartheta}^{-}\left(x_{\vartheta, 1}^{-}\right)^{(2 a-2)} v \neq 0 .
$$

Using (2.1.1) we get

$$
\left(x_{\vartheta}^{+}\right)^{(2 a-2)} x_{\vartheta}^{-}\left(x_{\vartheta, 1}^{-}\right)^{(2 a-2)} v=\left(x_{\vartheta}^{-}\left(x_{\vartheta}^{+}\right)^{(2 a-2)}+\left(x_{\vartheta}^{+}\right)^{(2 a-3)}\right)\left(x_{\vartheta, 1}^{-}\right)^{(2 a-2)} v .
$$

Lemma 2.1.1 together with step (4.1) will again imply that the right-hand side is zero. This completes the proof. 
5.3. Existence of Demazure flag. If $\mathfrak{g}$ is simply laced, Theorem 1.5.2(b) follows immediately from part (a) with $k=1$. Thus, assume from now on that $\mathfrak{g}$ is not simply laced and recall the notation introduced in Section 2.4.

Given $\lambda \in P^{+}$, let $\mu=\bar{\lambda} \in P_{\text {sh }}^{+}$and $v$ be the image of 1 in $W_{\mathbb{C}}^{c}(\lambda)$. Consider $W_{\mathbb{C}}^{\text {sh }}:=U\left(\mathfrak{g}_{\mathrm{sh}}[t]\right) v$ and $W_{\mathbb{Z}}^{\text {sh }}:=U_{\mathbb{Z}}\left(\mathfrak{g}_{\mathrm{sh}}[t]\right) v$. By [Naoi 2012, Lemma 4.17], there is an isomorphism of $U\left(\mathfrak{g}_{\text {sh }}[t]\right)$-modules $W_{\mathbb{C}}^{\text {sh }} \cong D_{\mathbb{C}}(1, \mu)$. By Corollary 3.3.3, $W_{\mathbb{Z}}^{\text {sh }}$ is an integral form of $W_{\mathbb{C}}^{c}(\mu) \cong D_{\mathbb{C}}(1, \mu)$. Hence, we have an isomorphism of $U_{\mathbb{Z}}\left(\mathfrak{g}_{\mathrm{sh}}[t]\right)$-modules $W_{\mathbb{Z}}^{\mathrm{sh}} \cong D_{\mathbb{Z}}(1, \mu)$.

Since $\mathfrak{g}_{\mathrm{sh}}$ is of type $A$, Theorem 4.4.1 implies that there exist $k>0, \mu_{1}, \ldots, \mu_{k} \in$ $P_{\text {sh }}^{+}, m_{1}, \ldots, m_{k} \in \mathbb{Z}_{\geq 0}$, and a filtration of $U_{\mathbb{Z}}\left(\mathfrak{g}_{\mathrm{sh}}[t]\right)$-modules $0=D_{0} \subseteq D_{1} \subseteq$ $\cdots \subseteq D_{k}=W_{\mathbb{Z}}^{\text {sh }}$, such that $D_{j}$ and $D_{j} / D_{j-1}$ are free $\mathbb{Z}$-modules, and $D_{j} / D_{j-1} \cong$ $D_{\mathbb{Z}}\left(r^{\vee}, \mu_{j}, m_{j}\right)$ for all $j=1, \ldots, k$. In particular,

$W_{\mathbb{Z}}^{\text {sh }} / D_{j}$ is a free $\mathbb{Z}$-module for all $j=0, \ldots, k$.

Set $\lambda_{j}=\eta_{\lambda}\left(\mu_{j}\right) \in P^{+}$where $\eta_{\lambda}$ is defined in (2.4.1), $W_{\mathbb{Z}}^{j}=U_{\mathbb{Z}}(\mathfrak{g}[t]) D_{j}$ and $W_{\mathbb{F}}^{j}=\mathbb{F} \otimes_{\mathbb{Z}} W_{\mathbb{Z}}^{j}$. It is easy to see that we have $0=W_{\mathbb{F}}^{0} \subseteq W_{\mathbb{F}}^{1} \subseteq \cdots \subseteq W_{\mathbb{F}}^{k}$, and $\lambda_{k}=\lambda$ since $\mu_{k}=\mu$. Hence, we are left to show that

$$
W_{\mathbb{F}}^{j} / W_{\mathbb{F}}^{j-1} \cong D_{\mathbb{F}}\left(1, \lambda_{j}, m_{j}\right) \text { for all } j=1, \ldots, k \text {, and } W_{\mathbb{F}}^{k} \cong W_{\mathbb{F}}^{c}(\lambda) \text {. }
$$

Notice that $W_{\mathbb{Z}}^{k}=U_{\mathbb{Z}}(\mathfrak{g}[t]) v$. Then Corollary 3.3.3 implies that $W_{\mathbb{Z}}^{k}$ is an integral form of $W_{\mathbb{C}}^{c}(\lambda)$. Since $\mathbb{Z}$ is a PID and $W_{\mathbb{Z}}^{k}$ is a finitely generated, free $\mathbb{Z}$-module, it follows that $W_{\mathbb{Z}}^{j}$ is a free $\mathbb{Z}$-module of finite rank for all $j=1, \ldots, k$. Set $W_{\mathbb{C}}^{j}=U(\mathfrak{g}[t]) D_{j}$. It follows from [Naoi 2012, Proposition 4.18] (which is Theorem 1.5.2(b) in characteristic zero) that $W_{\mathbb{C}}^{j} / W_{\mathbb{C}}^{j-1} \cong D_{\mathbb{C}}\left(1, \lambda_{j}, m_{j}\right)$ for all $j=1, \ldots, k$. Moreover, since $W_{\mathbb{C}}^{j} \cong \mathbb{C} \otimes_{\mathbb{Z}} W_{\mathbb{Z}}^{j}$, we have

$$
\mathbb{C} \otimes_{\mathbb{Z}}\left(W_{\mathbb{Z}}^{j} / W_{\mathbb{Z}}^{j-1}\right) \cong\left(W_{\mathbb{C}}^{j} / W_{\mathbb{C}}^{j-1}\right) \cong D_{\mathbb{C}}\left(1, \lambda_{j}, m_{j}\right) .
$$

Therefore, $W_{\mathbb{Z}}^{j} / W_{\mathbb{Z}}^{j-1}$ is a finitely generated $\mathbb{Z}$-module of rank $\operatorname{dim}\left(D_{\mathbb{C}}\left(1, \lambda_{j}, m_{j}\right)\right)$ for all $j=1, \ldots, k$. Since $W_{\mathbb{F}}^{j} / W_{\mathbb{F}}^{j-1} \cong \mathbb{F} \otimes_{\mathbb{Z}}\left(W_{\mathbb{Z}}^{j} / W_{\mathbb{Z}}^{j-1}\right)$, it follows that

$$
\operatorname{dim}\left(W_{\mathbb{E}}^{j} / W_{\mathbb{F}}^{j-1}\right) \geq \operatorname{dim}\left(D_{\mathbb{C}}\left(1, \lambda_{j}, m_{j}\right)\right)=\operatorname{dim}\left(D_{\mathbb{E}}\left(1, \lambda_{j}, m_{j}\right)\right) .
$$

Now, let $v_{j} \in D_{j}$ be as in Theorem 4.4.1, $w$ be the image of $v$ in $W_{\mathbb{F}}^{k}, u_{j} \in U_{\mathbb{Z}}\left(\mathfrak{n}_{\mathrm{sh}}^{-}[t]\right)$ be such that $v_{j}=u_{j} v$, and $w_{j}=u_{j} w$. It follows that

$$
W_{\mathbb{Z}}^{j}=\sum_{n \leq j} U_{\mathbb{Z}}(\mathfrak{g}[t]) v_{n} \quad \text { and } \quad W_{\mathbb{F}}^{j}=\sum_{n \leq j} U_{\mathbb{F}}(\mathfrak{g}[t]) w_{n} .
$$

We will show that the image $\bar{w}_{j}$ of $w_{j}$ in $W_{\mathbb{F}}^{j} / W_{\mathbb{F}}^{j-1}$ satisfies the relations described in Proposition 5.2.1, which implies that $W_{\mathbb{F}}^{j} / W_{\mathbb{F}}^{j-1}$ is a quotient of $D_{\mathbb{F}}\left(1, \lambda_{j}, m_{j}\right)$ and, hence, $W_{\mathbb{F}}^{j} / W_{\mathbb{F}}^{j-1} \cong D_{\mathbb{F}}\left(1, \lambda_{j}, m_{j}\right)$ for all $j=1, \ldots, k$. 
By construction, $v_{j}$ is a weight vector of weight $\lambda_{j}$ and degree $m_{j}$, and so is $w_{j}$. Since $D_{j} / D_{j-1} \cong D_{\mathbb{Z}}\left(r^{\vee}, \mu_{j}, m_{j}\right)$, it follows that

$$
U_{\mathbb{F}}\left(\mathfrak{n}_{\mathrm{sh}}^{+}[t]\right)^{0} \bar{w}_{j}=U_{\mathbb{F}}\left(\mathfrak{h}_{\mathrm{sh}}[t]_{+}\right)^{0} \bar{w}_{j}=0 \quad \text { and } \quad\left(x_{\alpha, s}^{-}\right)^{(k)} \bar{w}_{j}=0
$$

for all $\alpha \in R_{\mathrm{sh}}^{+}, s \geq 0, k>\max \left\{0, \lambda\left(h_{\alpha}\right)-s r^{\vee}\right\}, j=1, \ldots, k$. Thus, it remains to show that

$$
\left(x_{\alpha, s}^{+}\right)^{(m)} \bar{w}_{j}=\Lambda_{i, r} \bar{w}_{j}=\left(x_{\alpha}^{-}\right)^{(k)} \bar{w}_{j}=0
$$

for all $\alpha \in R^{+} \backslash R_{\mathrm{sh}}^{+}, s \geq 0, r, m>0, k>\lambda_{j}\left(h_{i}\right), j=1, \ldots, k$. Since,

$$
\lambda_{j}+m \alpha \notin \lambda-Q^{+} \text {for all } \alpha \in R^{+} \backslash R_{\mathrm{sh}}^{+}, m>0,
$$

we get $\left(x_{\alpha, s}^{+}\right)^{(m)} w_{j}=0$ for all $m>0, s \geq 0$. In particular, it follows that $\bar{w}_{j}$ is a highest-weight vector of weight $\lambda_{j}$ and, hence, $\left(x_{\alpha}^{-}\right)^{(k)} \bar{w}_{j}=0$ for all $\alpha \in R^{+}$, $k>\lambda\left(h_{\alpha}\right)$. Finally, we show that

$$
\Lambda_{i, r} \bar{w}_{j}=0 \text { for all } i \in I \backslash I_{\mathrm{sh}}, r>0, j=1, \ldots, k .
$$

Observe that

$$
\Lambda_{i, r} u_{j} \in U_{\mathbb{Z}}\left(\mathfrak{n}_{\mathrm{sh}}^{-}\right) U_{\mathbb{Z}}\left(\mathfrak{h}[t]_{+}\right) .
$$

In particular, $\Lambda_{i, r} v_{j} \in W_{\mathbb{Z}}^{\text {sh }} \cap W_{\mathbb{Z}}^{j}$. We will show that $\Lambda_{i, r} v_{j} \in D_{j-1}$ which implies (5.3.3). Let $y_{j} \in U_{\mathbb{Z}}\left(\mathfrak{n}_{\mathrm{sh}}^{-}\right)$be such that $\Lambda_{i, r} u_{j}=y_{j}$ modulo $U_{\mathbb{Z}}\left(\mathfrak{n}_{\mathrm{sh}}^{-}\right) U_{\mathbb{Z}}\left(\mathfrak{h}[t]_{+}\right)^{0}$. Thus, we want to show that

$$
y_{j} v \in D_{j-1} .
$$

We prove this recursively on $j=1, \ldots, k$. Notice that since $\mathbb{C} \otimes_{\mathbb{Z}}\left(W_{\mathbb{Z}}^{j} / W_{\mathbb{Z}}^{j-1}\right) \cong$ $D_{\mathbb{C}}\left(1, \lambda_{j}, m_{j}\right)$, there exists $n_{j} \in \mathbb{Z}_{>0}$ such that $n_{j} y_{j} v \in W_{\mathbb{Z}}^{j-1}, j=1, \ldots, k$. In particular, since $W_{\mathbb{Z}}^{0}=0$ and $W_{\mathbb{Z}}^{1}$ is a torsion-free $\mathbb{Z}$-module, (5.3.4) follows for $j=1$. Next, we show that (5.3.4) implies

$$
W_{\mathbb{Z}}^{j} \cap W_{\mathbb{Z}}^{\mathrm{sh}}=D_{j} .
$$

Indeed, it follows from (5.3.2) and (5.3.4) that

$$
W_{\mathbb{Z}}^{j}=U_{\mathbb{Z}}\left(\mathfrak{n}^{-}[t]\right) U_{\mathbb{Z}}\left(\mathfrak{g}_{\mathrm{sh}}[t]\right) v_{j}+W_{\mathbb{Z}}^{j-1} .
$$

Since $U_{\mathbb{Z}}\left(\mathfrak{h}_{\mathrm{sh}}[t]_{+}\right)^{0} U_{\mathbb{Z}}\left(\mathfrak{n}_{\mathrm{sh}}^{+}\right)^{0} v_{j} \in D_{j-1}$ and, by the induction hypothesis, $W_{\mathbb{Z}}^{j-1} \cap$ $W_{\mathbb{Z}}^{\text {sh }}=D_{j-1}$, (5.3.5) follows by observing that

$$
\left(U_{\mathbb{Z}}\left(\mathfrak{n}^{-}[t]\right) v_{j}\right) \cap W_{\mathbb{Z}}^{\text {sh }} \subseteq D_{j}
$$

(which is easily verified by weight considerations). Finally, observe that since $n_{j+1} y_{j+1} v$ is in $W_{\mathbb{Z}}^{j} \cap W_{\mathbb{Z}}^{\text {sh }}=D_{j}$, (5.3.1) implies that $y_{j+1} v \in D_{j}$. Thus, (5.3.5) for $j$ implies (5.3.4) for $j+1$ and the recursive step is proved. 
Remark 5.3.1. It follows from the above that $W_{\mathbb{F}}^{j} / W_{\mathbb{F}}^{j-1} \cong D_{\mathbb{F}}\left(1, \lambda_{j}, m_{j}\right)$ for any field $\mathbb{F}$. Hence, $W_{\mathbb{Z}}^{j} / W_{\mathbb{Z}}^{j-1}$ must be isomorphic to $D_{\mathbb{Z}}\left(1, \lambda_{j}, m_{j}\right)$ for all $j=1, \ldots, k$.

It remains to show that $W_{\mathbb{F}}^{k} \cong W_{\mathbb{F}}^{c}(\lambda)$. Since Theorem 3.3.4(c) implies that we have a projection $W_{\mathbb{F}}^{c}(\lambda) \rightarrow W_{\mathbb{F}}^{k}$ of $U_{\mathbb{F}}(\mathfrak{g}[t])$-modules, it suffices to show that $\operatorname{dim}\left(W_{\mathbb{F}}^{c}(\lambda)\right) \leq \operatorname{dim}\left(W_{\mathbb{F}}^{k}\right)$. This follows if we show that there exists a filtration $0=$ $\tilde{W}_{\mathbb{F}}^{0} \subseteq \tilde{W}_{\mathbb{F}}^{1} \subseteq \cdots \subseteq \tilde{W}_{\mathbb{F}}^{k}=W_{\mathbb{F}}^{c}(\lambda)$ such that $\tilde{W}_{\mathbb{F}}^{j} / \tilde{W}_{\mathbb{F}}^{j-1}$ is a quotient of $D_{\mathbb{F}}\left(1, \lambda_{j}, m_{j}\right)$ for all $j=1, \ldots, k$. Let $w^{\prime}$ be the image of 1 in $W_{\mathbb{F}}^{c}(\lambda), w_{j}^{\prime}=u_{j} w^{\prime} \in W_{\mathbb{F}}^{c}(\lambda), \tilde{W}_{\mathbb{F}}^{j}:=$ $\sum_{n \leq j} U_{\mathbb{F}}(\mathfrak{g}[t]) w_{n}^{\prime} \subseteq W_{\mathbb{F}}^{c}(\lambda)$, and $\bar{w}_{j}^{\prime}$ be the image of $w_{j}^{\prime}$ in $\tilde{W}_{\mathbb{F}}^{j} / \tilde{W}_{\mathbb{F}}^{j-1}$. Observe that $\tilde{W}_{\mathbb{F}}^{k}=W_{\mathbb{F}}^{c}(\lambda)$. We need to show that $\bar{w}_{j}^{\prime}$ satisfies the defining relations of $D_{\mathbb{F}}\left(1, \lambda_{j}\right)$ listed in Proposition 5.2.1. Let $\tilde{D}_{j}=\mathbb{F} \otimes_{\mathbb{Z}} D_{j}$ and $D_{j}^{\prime}=\sum_{n \leq j} U_{\mathbb{F}}\left(\mathfrak{g}_{\mathrm{sh}}[t]\right) w_{n}^{\prime}$. Notice that $D_{k}^{\prime}$ is a quotient of $W_{\mathbb{F}}^{c}(\mu) \cong \tilde{D}_{k}$ and let $\pi: \tilde{D}_{k} \rightarrow D_{k}^{\prime}$ be a $U_{\mathbb{F}}\left(\mathfrak{g}_{\mathrm{sh}}[t]\right)$-module epimorphism such that $v_{k} \mapsto w_{k}^{\prime}$ (we keep denoting the image of $v_{j}$ in $\tilde{D}_{j}$ by $\left.v_{j}\right)$. In particular, $w_{j}^{\prime}=\pi\left(v_{j}\right)$ and $\pi$ induces an epimorphism $\tilde{D}_{j} \rightarrow D_{j}^{\prime}$ for all $j=1, \ldots, k$. Hence,

$$
x w_{j}^{\prime} \in D_{j-1}^{\prime} \text { for all } x \in U_{\mathbb{Z}}\left(\mathfrak{g}_{\mathrm{sh}}[t]\right) \text { such that } x v_{j} \in D_{j-1} .
$$

This immediately implies that

$$
U_{\mathbb{F}}\left(\mathfrak{n}_{\mathrm{sh}}^{+}[t]\right)^{0} \bar{w}_{j}^{\prime}=U_{\mathbb{F}}\left(\mathfrak{h}[t]_{+}\right)^{0} \bar{w}_{j}^{\prime}=0 \quad \text { and } \quad\left(x_{\alpha, s}^{-}\right)^{(k)} \bar{w}_{j}^{\prime}=0
$$

for all $\alpha \in R_{\mathrm{sh}}^{+}, s \geq 0, k>\max \left\{0, \lambda\left(h_{\alpha}\right)-s r^{\vee}\right\}, j=1, \ldots, k$. Note that (5.3.4) has been used here. The relations

$$
\left(x_{\alpha, s}^{+}\right)^{(m)} \bar{w}_{j}^{\prime}=\left(x_{i}^{-}\right)^{(k)} \bar{w}_{j}^{\prime}=0
$$

for all $\alpha \in R^{+} \backslash R_{\mathrm{sh}}^{+}, i \in I \backslash I_{\mathrm{sh}}, s \geq 0, m>0, k>\lambda_{j}\left(h_{i}\right), j=1, \ldots, k$ follow from (5.3.2) as before.

\subsection{The isomorphism between local Weyl modules and graded local Weyl mod-} ules. We now prove Theorem 1.5.2(c). Recall the definition of the automorphism $\varphi_{a}$ of $U_{\mathbb{F}}(\mathfrak{g}[t])$ from Section 2.2. In particular, let $\tilde{a} \in \mathbb{A}^{\times}$be such that its image in $\mathbb{F}$ is $a$. Denote by $\varphi_{a}^{*}\left(W_{\mathbb{F}}\left(\boldsymbol{\omega}_{\lambda, a}\right)\right)$ the pull-back of $W_{\mathbb{F}}\left(\boldsymbol{\omega}_{\lambda, a}\right)$ (regarded as a $U_{\mathbb{F}}(\mathfrak{g}[t])$-module) by $\varphi_{a}$.

Notice that

$$
\operatorname{dim} W_{\mathbb{F}}\left(\boldsymbol{\omega}_{\lambda, a}\right)=\operatorname{dim} W_{\mathbb{K}}\left(\boldsymbol{\omega}_{\lambda, \tilde{a}}\right)=\operatorname{dim} W_{\mathbb{K}}^{c}(\lambda)=\operatorname{dim} W_{\mathbb{F}}^{c}(\lambda) .
$$

Here, the first equality follows from (1.5.4), the second from (3.4.1) (with $\mathbb{F}=$ $\mathbb{K})$ together with Proposition 3.4.1, and the third from Corollary 1.5.3. Since $\operatorname{dim} \varphi_{a}^{*}\left(W_{\mathbb{F}}\left(\boldsymbol{\omega}_{\lambda, a}\right)\right)=\operatorname{dim} W_{\mathbb{F}}\left(\boldsymbol{\omega}_{\lambda, a}\right)$, Theorem 1.5.2(c) follows if we show that $\varphi_{a}^{*}\left(W_{\mathbb{F}}\left(\boldsymbol{\omega}_{\lambda, a}\right)\right)$ is a quotient of $W_{\mathbb{F}}^{c}(\lambda)$.

Let $w \in W_{\mathbb{F}}\left(\boldsymbol{\omega}_{\lambda, a}\right)_{\lambda} \backslash\{0\}$ and use the symbol $w_{a}$ to denote $w$ when regarded as an element of $\varphi_{a}^{*}\left(W_{\mathbb{F}}\left(\boldsymbol{\omega}_{\lambda, a}\right)\right)$. Since $W_{\mathbb{F}}\left(\boldsymbol{\omega}_{\lambda, a}\right)=U_{\mathbb{F}}(\mathfrak{g}[t]) w$ and $\varphi_{a}$ is an automorphism 
of $U_{\mathbb{F}}(\mathfrak{g}[t])$, it follows that $\varphi_{a}^{*} W_{\mathbb{F}}\left(\boldsymbol{\omega}_{\lambda, a}\right)=U_{\mathbb{F}}(\mathfrak{g}[t]) w_{a}$. Thus, we need to show that $w_{a}$ satisfies the defining relations (1.5.1) of $W_{\mathbb{F}}^{c}(\lambda)$. Since $\varphi_{a}$ fixes every element of $U_{\mathbb{F}}(\mathfrak{g}), w_{a}$ is a vector of weight $\lambda$ annihilated by $\left(x_{\alpha}^{-}\right)^{(k)}$ for all $\alpha \in$ $R^{+}, k>\lambda\left(h_{\alpha}\right)$. Equation (2.2.6) implies that $\varphi_{a}$ maps $U_{\mathbb{F}}\left(\mathfrak{n}^{+}[t]\right)$ to itself and, hence, $U_{\mathbb{F}}\left(\mathfrak{n}^{+}[t]\right)^{0} w_{a}=0$. Therefore, it remains to show that

$$
U_{\mathbb{F}}\left(\mathfrak{h}[t]_{+}\right)^{0} w_{a}=0 .
$$

To show this, let $v$ be in $W_{\mathbb{K}}\left(\boldsymbol{\omega}_{\lambda, \tilde{a}}\right)_{\lambda} \backslash\{0\}$ and $L=U_{\mathbb{A}}(\mathfrak{g}[t]) v$. By (1.5.4), $\mathbb{F} \otimes_{\mathbb{A}} L \cong$ $W_{\mathbb{F}}\left(\boldsymbol{\omega}_{\lambda, a}\right)$. In particular, the action of $U_{\mathbb{F}}\left(\mathfrak{h}[t]_{+}\right)^{0}$ on $\varphi_{a}^{*}\left(W_{\mathbb{E}}\left(\boldsymbol{\omega}_{\lambda, a}\right)\right)$ is obtained from the action of $U_{\mathbb{A}}\left(\mathfrak{h}[t]_{+}\right)^{0}$ on $\varphi_{\tilde{a}}^{*}\left(W_{\mathbb{K}}\left(\boldsymbol{\omega}_{\lambda, \tilde{a}}\right)\right)$ which, in turn, is obtained from the action of $U_{\mathbb{K}}\left(\mathfrak{h}[t]_{+}\right)^{0}$. Since $U_{\mathbb{K}}\left(\mathfrak{h}[t]_{+}\right)$is generated by $h_{i, r}, i \in I, r>0$, we are left to show that

$$
h_{i, r} v_{a}=0,
$$

where $v_{a}$ is the vector $v$ regarded as an element of $\varphi_{\tilde{a}}^{*}\left(W_{\mathbb{}}\left(\boldsymbol{\omega}_{\lambda, \tilde{a}}\right)\right)$. It is well known that the irreducible quotient of $W_{\mathbb{}}\left(\boldsymbol{\omega}_{\lambda, \tilde{a})}\right)$ is the evaluation module with evaluation parameter $\tilde{a}$ (see [Jakelić and Moura 2007, Section 3B]). Hence, $h_{i, s} v=\tilde{a}^{s} \lambda\left(h_{i}\right) v$ for all $i \in I, s \in \mathbb{Z}$. Using this, it follows that, for all $i \in I, r>0$, we have

$$
h_{i, r} v_{a}=\left(h_{i} \otimes(t-\tilde{a})^{r}\right) v=\sum_{s=0}^{r}\left(\begin{array}{l}
r \\
s
\end{array}\right)(-\tilde{a})^{s} h_{i, r-s} v=\lambda\left(h_{i}\right) \tilde{a}^{r} \sum_{s=0}^{r}\left(\begin{array}{l}
r \\
s
\end{array}\right)(-1)^{s} v=0 .
$$

5.5. A tensor product theorem. We say that $\boldsymbol{\omega}, \boldsymbol{\pi} \in \mathscr{P}_{\mathbb{F}}^{+}$are relatively prime if for all $i, j \in I$ the polynomials $\omega_{i}(u)$ and $\pi_{j}(u)$ are relatively prime in $\mathbb{F}[u]$. The goal of this subsection is to prove the following theorem from which we will deduce Theorem 1.5.2(d).

Theorem 5.5.1. Suppose $\omega, \pi \in \mathscr{P}_{\mathbb{F}}^{+}$are relatively prime and that $V$ and $W$ are quotients of $W_{\mathbb{F}}(\boldsymbol{\omega})$ and $W_{\mathbb{E}}(\boldsymbol{\pi})$, respectively. Then $V \otimes W$ is generated by its top weight space.

Theorem 5.5.1 was proved in [Chari and Pressley 2001] in the case $\mathbb{F}=\mathbb{C}$. Although the proof we present here follows the same general lines, there are several extra technical issues to be taken care of arising from the fact that $U_{\mathbb{C}}(\tilde{\mathfrak{g}})$ is generated by $x_{\alpha, r}^{ \pm}, \alpha \in R^{+}, r \in \mathbb{Z}$, while, in the case of $U_{\mathbb{F}}(\tilde{\mathfrak{g}})$, we also need arbitrarily large divided powers of these elements. We start the proof by establishing a few technical lemmas. Recall the definition of $X_{\alpha, m, s}^{-}(u)$ in Section 2.1 and set

$$
X_{\alpha ; s}^{-}(u)=X_{\alpha, 1, s+1}^{-}(u)
$$

To shorten notation, we shall often write $X_{\alpha ; s}^{-}$instead of $X_{\alpha ; s}^{-}(u)$. 
Fix $\omega \in \mathscr{P}^{+}$and let $w$ be a highest- $\ell$-weight vector of $W_{\mathbb{F}}(\omega)$. Given $\beta \in R^{+}$, define $\omega_{\beta}(u) \in \mathbb{E}[u]$ by

$$
\boldsymbol{\omega}_{\beta}(u) w=\Lambda_{\beta}(u) w .
$$

One can easily check (see [Chari and Pressley 2001, Lemma 3.1]) that if $\vartheta$ is the highest short root of $\mathfrak{g}$ and $\beta \in R^{+}$, then there exists $\boldsymbol{\omega}_{\vartheta, \beta} \in \mathscr{P}^{+}$such that

$$
\omega_{\vartheta}=\omega_{\beta} \omega_{\vartheta, \beta} .
$$

Lemma 5.5.2. For all $\beta \in R^{+}, k, l, s \in \mathbb{Z}, 0 \leq l \leq k, k>\lambda\left(h_{\beta}\right)$, we have

$$
\left(\boldsymbol{\omega}_{\vartheta} X_{\beta ; s}^{-{ }^{(k-l)}}\right)_{k+\operatorname{deg}\left(\omega_{\vartheta, \beta}\right)} w=0 .
$$

Proof. We will need the following particular case of Lemma 2.1.1:

$$
\left(x_{\beta,-s}^{+}\right)^{(l)}\left(x_{\beta, s+1}^{-}\right)^{(k)}=(-1)^{l}\left(\left(X_{\beta ; s}^{-}(u)\right)^{(k-l)} \Lambda_{\beta}(u)\right)_{k} \quad \bmod U_{\mathbb{Z}}(\tilde{\mathfrak{g}}) U_{\mathbb{Z}}\left(\tilde{\mathfrak{n}}^{+}\right)^{0}
$$

for all $k, l, s \in \mathbb{Z}, 0 \leq l \leq k$. It follows from (5.5.1) and the definition of $\omega_{\beta}$ that

$$
\left(\omega_{\beta} X_{\beta ; s}^{-(k-l)}\right)_{k} w=0 \text { for all } k, l, s \in \mathbb{Z}, 0 \leq l \leq k, k>\lambda\left(h_{\beta}\right) .
$$

Hence, for such $k, l, s$, we have

$$
\begin{aligned}
&\left(\boldsymbol{\omega}_{\vartheta} X_{\beta ; s}^{-}{ }^{(k-l)}\right)_{k+\operatorname{deg}\left(\boldsymbol{\omega}_{\vartheta, \beta}\right)} w=\left(\boldsymbol{\omega}_{\vartheta, \beta} \boldsymbol{\omega}_{\beta} X_{\beta ; s}^{-}{ }^{(k-l)}\right)_{k+\operatorname{deg}\left(\boldsymbol{\omega}_{\vartheta, \beta}\right)} w \\
&=\sum_{j=0}^{\operatorname{deg}\left(\boldsymbol{\omega}_{\vartheta, \beta}\right)}\left(\boldsymbol{\omega}_{\vartheta, \beta}\right)_{j}\left(\boldsymbol{\omega}_{\beta} X_{\beta ; s}^{-}{ }^{(k-l)}\right)_{k+\operatorname{deg}\left(\boldsymbol{\omega}_{\vartheta, \beta}\right)-j} w=0,
\end{aligned}
$$

where the last equality follows from (5.5.2) since $k+\operatorname{deg}\left(\boldsymbol{\omega}_{\vartheta, \beta}\right)-j>\lambda\left(h_{\beta}\right)$.

Let $\mathscr{R}=R^{+} \times \mathbb{Z} \times \mathbb{Z}_{\geq 0}$ and $\Xi$ be the set of functions $\xi: \mathbb{N} \rightarrow \mathscr{R}$ given by $j \mapsto \xi_{j}=\left(\beta_{j}, s_{j}, k_{j}\right)$, such that $k_{j}=0$ for all $j$ sufficiently large. Define the degree of $\xi$ to be $d(\xi)=\sum_{j} k_{j}$. Let $\Xi_{d}$ be the subset of functions of degree $d$ and $\Xi_{d}^{<}=\bigcup_{d^{\prime}<d} \Xi_{d^{\prime}}$. Given $\xi \in \Xi$ such that $\xi_{j}=\left(\beta_{j}, s_{j}, k_{j}\right)$ for all $j \in \mathbb{N}$ and $k_{j}=0$ for $j>m$, set

$$
x^{\xi}=\left(x_{\beta_{1}, s_{1}}^{-}\right)^{\left(k_{1}\right)} \cdots\left(x_{\beta_{m}, s_{m}}^{-}\right)^{\left(k_{m}\right)} \text { and } \quad w^{\xi}=x^{\xi} w .
$$

It will be convenient to write $\operatorname{deg}\left(w^{\xi}\right)=d(\xi)=\operatorname{deg}\left(x^{\xi}\right)$. The next lemma is an easy consequence of [Mitzman 1985, Lemma 4.2.13].

Lemma 5.5.3. Let $\alpha$ be in $R^{+}, s \in \mathbb{Z}, d, k$ be in $\mathbb{Z}_{\geq 0}$, and $\xi$ be in $\Xi_{d}$. Then $x^{\xi}\left(x_{\alpha, s}^{-}\right)^{(k)}$ is in the span of

$$
\left\{\left(x_{\alpha, s}^{-}\right)^{(k)} x^{\xi}\right\} \cup\left\{x^{\varsigma}: \varsigma \in \Xi_{d+k}^{<}\right\} .
$$


Lemma 5.5.4. Let $\beta$ be in $R^{+}, k$ in $\mathbb{Z}, d, r, s$ in $\mathbb{Z}_{\geq 0}, r \leq s, s>\lambda\left(h_{\beta}\right)$ and $\xi$ in $\Xi_{d}$. Then $\left(\boldsymbol{\omega}_{\vartheta} X_{\beta ; k}^{-}{ }^{(r)}\right)_{s} w^{\xi}$ is in the span of vectors of the form $w^{\varsigma}$ with $\varsigma \in \Xi_{r+d}^{<}$. Proof. If $d=0$, it follows from (5.5.2) that $\left(\omega_{\vartheta} X_{\beta ; k}^{-}{ }^{(r)}\right)_{s} w^{\xi}=0$, which proves the lemma in this case. We now proceed by induction on $d$. Thus, let $d>0$ and write $w^{\xi}=\left(x_{\beta_{1}, s_{1}}^{-}\right)^{\left(k_{1}\right)} \cdots\left(x_{\beta_{l}, s_{l}}^{-}\right)^{\left(k_{l}\right)} w$ with $k_{1} \neq 0$. Let also $\xi^{\prime} \in \Xi$ be such that

$$
\xi_{j}^{\prime}= \begin{cases}\xi_{j}, & \text { if } j \neq 1, \\ \left(\beta_{1}, s_{1}, 0\right), & \text { if } j=1 .\end{cases}
$$

Then, by Lemma 5.5.3, we have

$$
\left(\boldsymbol{\omega}_{\vartheta} X_{\beta ; k}^{-(r)}\right)_{s} w^{\xi}=\left(\boldsymbol{\omega}_{\vartheta} X_{\beta ; k}^{-}{ }^{(r)}\right)_{s}\left(x_{\beta_{1}, s_{1}}^{-}\right)^{\left(k_{1}\right)} w^{\xi^{\prime}}=\left(x_{\beta_{1}, s_{1}}^{-{ }^{\left(k_{1}\right)}}\left(\boldsymbol{\omega}_{\vartheta} X_{\beta ; k}^{-}{ }^{(r)}\right)_{s} w^{\xi^{\prime}}+X w^{\xi^{\prime}}\right.
$$

where $X$ is in the span of $\left\{x^{\varsigma}: \varsigma \in \Xi_{r+k_{1}}^{<}\right\}$. In particular, $X w^{\xi^{\prime}}$ is in the span of vectors of the desired form. Since $d\left(\xi^{\prime}\right)=d-k_{1}<d$, the induction hypothesis implies that $\left(\boldsymbol{\omega}_{\vartheta} X_{\beta ; k}^{-}{ }^{(r)}\right)_{s} w^{\xi^{\prime}}$ is in the span of vectors associated to elements of $\Xi_{r+d-k_{1}}^{<}$. Therefore, $\left(x_{\beta_{1}, s_{1}}^{-}\right)^{\left(k_{1}\right)}\left(\boldsymbol{\omega}_{\vartheta} X_{\beta ; k}^{-}{ }^{(r)}\right)_{s} w^{\xi^{\prime}}$ is in the span of vectors associated to elements of $\Xi_{r+d}^{<}$as desired.

Proof of Theorem 5.5.1. Let $w_{\omega}$ and $w_{\pi}$ be highest- $\ell$-weight vectors for $V$ and $W$, respectively. Let also

$$
M=U_{\mathbb{F}}(\tilde{\mathfrak{g}})\left(w_{\omega} \otimes w_{\pi}\right)=U_{\mathbb{F}}\left(\tilde{\mathfrak{n}}^{-}\right)\left(w_{\omega} \otimes w_{\pi}\right) .
$$

Our goal is to show that $M=V \otimes W$. Since the vectors $w_{\omega}^{\xi} \otimes w_{\pi}^{\xi^{\prime}}, \xi, \xi^{\prime} \in \Xi$ span $V \otimes W$, it suffices to show that these vectors are in $M$. We do this by induction on $d(\xi)+d\left(\xi^{\prime}\right)$ which obviously starts when $d(\xi)+d\left(\xi^{\prime}\right)=0$ since, in this case, $w_{\omega}^{\xi} \otimes w_{\pi}^{\xi^{\prime}}=w_{\omega} \otimes w_{\pi}$.

Let $n \geq 0$, and suppose, by induction hypothesis, that

$$
w_{\omega}^{\xi} \otimes w_{\pi}^{\xi^{\prime}} \in M \text { for all } \xi, \xi^{\prime} \in \Xi \text { such that } d(\xi)+d\left(\xi^{\prime}\right) \leq n .
$$

In order to complete the induction step, it suffices to show that

$$
w_{\omega}^{\xi} \otimes\left(x_{\beta, l}^{-}\right)^{(r)} w_{\pi}^{\xi^{\prime}} \in M \quad \text { and } \quad\left(\left(x_{\beta, l}^{-}\right)^{(r)} w_{\omega}^{\xi}\right) \otimes w_{\pi}^{\xi^{\prime}} \in M
$$

for all $\beta \in R^{+}, r, l \in \mathbb{Z}, r \geq 1, \xi, \xi^{\prime} \in \Xi$, such that $d(\xi)+d\left(\xi^{\prime}\right)+r=n+1$. We prove (5.5.5) by a further induction on $r \geq 1$. Henceforth we fix $\beta \in R^{+}$.

Observe that the hypothesis on $\omega$ and $\pi$ implies that $\boldsymbol{\omega}_{\vartheta}$ and $\boldsymbol{\pi}_{\vartheta}$ are relatively prime. Therefore, we can choose $R, S \in \mathbb{F}[u]$ such that

Set

$$
R \boldsymbol{\omega}_{\vartheta}+S \boldsymbol{\pi}_{\vartheta}=1 .
$$

$$
\delta=\operatorname{deg}\left(R \boldsymbol{\omega}_{\vartheta}\right)=\operatorname{deg}\left(S \boldsymbol{\pi}_{\vartheta}\right) \quad \text { and } \quad m=\max \left\{\operatorname{wt}(\boldsymbol{\omega})\left(h_{\beta}\right), \operatorname{wt}(\boldsymbol{\pi})\left(h_{\beta}\right)\right\} .
$$

We claim that for all $\xi \in \Xi$ and $k \in \mathbb{Z}$,

$$
\left(R \boldsymbol{\omega}_{\vartheta} X_{\beta ; k}^{-(r)}\right)_{s} w_{\omega}^{\xi} \in \operatorname{span}\left(\left\{w_{\omega}^{\varsigma}: \varsigma \in \Xi_{d(\xi)+r}^{<}\right\}\right) \text {for all } s>m+\delta .
$$


Indeed,

$$
\left(R \boldsymbol{\omega}_{\theta} X_{\beta ; k}^{-(r)}\right)_{s} w_{\omega}^{\xi}=\sum_{j=0}^{\operatorname{deg} R} R_{j}\left(\boldsymbol{\omega}_{\vartheta} X_{\beta ; k}^{-(r)}\right)_{s-j} w_{\omega}^{\xi}
$$

and, since $s-j>m+\delta-j \geq m+\operatorname{deg}\left(\boldsymbol{\omega}_{\vartheta}\right) \geq \operatorname{wt}(\boldsymbol{\omega})\left(h_{\beta}\right)$, the claim follows from Lemma 5.5.4. Similarly one proves that

$$
\left(S \pi_{\vartheta} X_{\beta ; k}^{-(r)}\right)_{s} w_{\pi}^{\xi} \in \operatorname{span}\left(\left\{w_{\pi}^{\varsigma}: \varsigma \in \Xi_{d(\xi)+r}^{<}\right\}\right) \text {for all } s>m+\delta .
$$

We are ready to start the proof of (5.5.5). Suppose $d(\xi)+d\left(\xi^{\prime}\right)=n$ and let $\ell>m+\delta$. Then

$$
\begin{aligned}
\left(R \boldsymbol{\omega}_{\vartheta} X_{\beta ; k}^{-}\right)_{\ell} & \left(w_{\omega}^{\xi} \otimes w_{\pi}^{\xi^{\prime}}\right) \\
= & \left(\left(R \boldsymbol{\omega}_{\vartheta} X_{\beta ; k}^{-}\right)_{\ell} w_{\omega}^{\xi}\right) \otimes w_{\pi}^{\xi^{\prime}}+w_{\omega}^{\xi} \otimes\left(\left(1-S \boldsymbol{\pi}_{\vartheta}\right) X_{\beta ; k}^{-}\right)_{\ell} w_{\boldsymbol{\pi}}^{\xi^{\prime}} \\
= & \left(\left(R \boldsymbol{\omega}_{\vartheta} X_{\beta ; k}^{-}\right)_{\ell} w_{\omega}^{\xi}\right) \otimes w_{\boldsymbol{\pi}}^{\xi^{\prime}}-w_{\omega}^{\xi} \otimes\left(S \boldsymbol{\pi}_{\vartheta} X_{\beta ; k}^{-}\right)_{\ell} w_{\pi}^{\xi^{\prime}}+w_{\omega}^{\xi} \otimes x_{\beta ; \ell+k}^{-} w_{\pi}^{\xi^{\prime}} .
\end{aligned}
$$

It follows from $(5.5 .6),(5.5 .7)$ and (5.5.4) that $\left(\left(R \omega_{\vartheta} X_{\xi^{\prime}}{ }_{\beta ; k}\right)_{\ell} w_{\omega}^{\xi}\right) \otimes w_{\pi}^{\xi^{\prime}}$ and $w_{\omega}^{\xi} \otimes$ $\left(S \boldsymbol{\pi}_{\vartheta} X_{\beta ; k}^{-}\right)_{\ell} w_{\pi}^{\xi^{\prime}}$ are in $M$. Since $\left(R \boldsymbol{\omega}_{\vartheta} X_{\beta ; k}^{-}\right)_{\ell}\left(w_{\omega}^{\xi} \otimes w_{\boldsymbol{\pi}}^{\xi^{\prime}}\right)$ is in $M$ by definition, it follows that $w_{\omega}^{\xi} \otimes x_{\beta ; \ell+k}^{-} w_{\pi}^{\xi^{\prime}}$ is in $M$ for all $k \in \mathbb{Z}$, which proves the first statement in (5.5.5) with $r=1$. The second statement is proved similarly by looking at $\left(S \boldsymbol{\pi}_{\vartheta} X_{\beta ; k}^{-}\right)_{\ell}\left(w_{\omega}^{\xi} \otimes w_{\pi}^{\xi^{\prime}}\right)$.

Let $r>1, \xi, \xi^{\prime} \in \Xi$ be such that $r+d(\xi)+d\left(\xi^{\prime}\right)=n+1$ and set $\ell=r \ell^{\prime}$ with $\ell^{\prime}$ such that $\ell>m+\delta$. Then

$$
\begin{aligned}
& \left(R \boldsymbol{\omega}_{\vartheta} X_{\beta ; k}^{-(r)}\right)_{\ell}\left(w_{\omega}^{\xi} \otimes w_{\pi}^{\xi^{\prime}}\right) \\
& =\left(\left(R \boldsymbol{\omega}_{\vartheta} X_{\beta ; k}^{-(r)}\right)_{\ell} w_{\omega}^{\xi}\right) \otimes w_{\pi}^{\xi^{\prime}}+w_{\boldsymbol{\pi}}^{\xi^{\prime}} \otimes\left(R \boldsymbol{\omega}_{\vartheta} X_{\beta ; k}^{-{ }^{(r)}}\right)_{\ell} w_{\boldsymbol{\pi}}^{\xi^{\prime}}+v \\
& =\left(\left(R \boldsymbol{\omega}_{\vartheta} X_{\beta ; k}^{-{ }^{(r)}}\right)_{\ell} w_{\omega}^{\xi}\right) \otimes w_{\pi}^{\xi^{\prime}}+w_{\omega}^{\xi} \otimes\left(\left(1-S \boldsymbol{\pi}_{\vartheta}\right) X_{\beta ; k}^{-{ }^{(r)}}\right)_{\ell} w_{\boldsymbol{\pi}}^{\xi^{\prime}}+v \\
& =\left(\left(R \boldsymbol{\omega}_{\vartheta} X_{\beta ; k}^{-(r)}\right)_{\ell} w_{\omega}^{\xi}\right) \otimes w_{\pi}^{\xi^{\prime}}-w_{\omega}^{\xi} \otimes\left(S \pi_{\vartheta} X_{\beta ; k}^{-{ }^{(r)}}\right)_{\ell} w_{\boldsymbol{\pi}}^{\xi^{\prime}} \\
& +w_{\omega}^{\xi} \otimes\left(X_{\beta ; k}^{-(r)}\right)_{\ell} w_{\pi}^{\xi^{\prime}}+v \\
& =\left(\left(R \boldsymbol{\omega}_{\vartheta} X_{\beta ; k}^{-}{ }^{(r)}\right)_{\ell} w_{\omega}^{\xi}\right) \otimes w_{\pi}^{\xi^{\prime}}-w_{\omega}^{\xi} \otimes\left(S \pi_{\vartheta} X_{\beta ; k}^{-}{ }^{(r)}\right)_{\ell} w_{\boldsymbol{\pi}}^{\xi^{\prime}} \\
& +w_{\omega}^{\xi} \otimes\left(x_{\beta, \ell^{\prime}+k}^{-}\right)^{(r)} w_{\pi}^{\xi^{\prime}}+w_{\omega}^{\xi} \otimes X w_{\pi}^{\xi^{\prime}}+v,
\end{aligned}
$$

where $v$ is in the span of vectors of the form

$$
\left(\prod_{i}\left(x_{\beta, s_{i}}^{-}\right)^{\left(a_{i}\right)} w_{\omega}^{\xi}\right) \otimes\left(\prod_{j}\left(x_{\beta, s_{j}}^{-}\right)^{\left(b_{j}\right)} w_{\pi}^{\xi^{\prime}}\right) \text { with } 1 \leq a_{i}, b_{j}<r, \sum_{i} a_{i}+\sum_{j} b_{j}=r,
$$

and $X$ is in the span of elements of the form

$$
\left(x_{\beta, s_{1}}^{-}\right)^{\left(r_{1}\right)}\left(x_{\beta, s_{2}}^{-}\right)^{\left(r_{2}\right)} \cdots\left(x_{\beta, s_{n}}^{-}\right)^{\left(r_{n}\right)} \text { with } r_{1}+\cdots+r_{n}=r, \quad 0<r_{j}<r .
$$


Again, $\left(R \omega_{\vartheta} X_{\beta ; k}^{-(r)}\right)_{\ell}\left(w_{\omega}^{\xi} \otimes w_{\pi}^{\xi^{\prime}}\right)$ is in $M$ by definition, while (5.5.6), (5.5.7), and (5.5.4), imply that

$$
\left(\left(R \boldsymbol{\omega}_{\vartheta} X_{\beta ; k}^{-(r)}\right)_{\ell} w_{\omega}^{\xi}\right) \otimes w_{\pi}^{\xi^{\prime}} \in M \quad \text { and } \quad w_{\omega}^{\xi} \otimes\left(S \boldsymbol{\pi}_{\vartheta} X_{\beta ; k}^{-}\right)_{\ell} w_{\pi}^{\xi^{\prime}} \in M .
$$

By induction hypothesis on $r$, it follows that $v$ and $w_{\omega}^{\xi} \otimes X w_{\pi}^{\xi^{\prime}}$ are in $M$, which then implies that $w_{\omega}^{\xi} \otimes\left(x_{\beta, \ell^{\prime}+k}^{-}\right)^{(r)} w_{\pi}^{\xi^{\prime}}$ is in $M$ for all $k \in \mathbb{Z}$, completing the proof of the first statement of (5.5.5). The second statement is proved similarly by looking at $\left(S \boldsymbol{\pi}_{\vartheta} X_{\beta ; k}^{-{ }^{(r)}}\right)_{\ell}\left(w_{\boldsymbol{\omega}}^{\xi} \otimes w_{\pi}^{\xi^{\prime}}\right)$.

5.6. The tensor product factorization of local Weyl modules. Theorem 1.5.2(d) clearly follows if we prove

$$
W_{\mathbb{F}}\left(\varpi_{1}\right) \otimes W_{\mathbb{F}}\left(\varpi_{2}\right) \cong W_{\mathbb{F}}\left(\varpi_{1} \varpi_{2}\right)
$$

whenever $\varpi_{1}, \varpi_{2} \in \mathscr{P}_{\mathbb{F}}^{+}$are relatively prime.

In order to show (5.6.1), let $w_{\varpi_{1}}$ and $w_{\varpi_{2}}$ be highest- $\ell$-weight vectors for $W_{\mathbb{F}}\left(\varpi_{1}\right)$ and $W_{\mathbb{F}}\left(\varpi_{2}\right)$, respectively. It is well known that $w_{\varpi_{1}} \otimes v_{\varpi_{2}}$ satisfies the defining relations of $W_{\mathbb{F}}\left(\varpi_{1} \varpi_{2}\right)$, so there exists a $U_{\mathbb{F}}(\tilde{\mathfrak{g}})$-module map $\phi: W_{\mathbb{F}}\left(\varpi_{1} \varpi_{2}\right) \rightarrow$ $W_{\mathbb{F}}\left(\varpi_{1}\right) \otimes W_{\mathbb{F}}\left(\varpi_{2}\right)$ that sends $w_{\varpi_{1} \varpi_{2}}$ to $w_{\varpi_{1}} \otimes w_{\varpi_{2}}$. Theorem 5.5.1 implies that $\phi$ is surjective. Hence, it suffices to show that

$$
\operatorname{dim}\left(W_{\mathbb{F}}\left(\varpi_{1} \varpi_{2}\right)\right)=\operatorname{dim}\left(W_{\mathbb{F}}\left(\varpi_{1}\right) \otimes W_{\mathbb{F}}\left(\varpi_{2}\right)\right) .
$$

In fact, recall from Remark 1.5.5 that there exist $\omega_{1}, \omega_{2} \in \mathscr{P}_{\mathbb{A}}^{\times}$such that $\varpi_{1}$ and $\varpi_{2}$ are the images of $\omega_{1}$ and $\omega_{2}$ in $\mathscr{P}_{\mathbb{F}}^{+}$, respectively. It then follows from (1.5.4) that

$$
\begin{aligned}
\operatorname{dim}\left(W_{\mathbb{F}}\left(\varpi_{1} \varpi_{2}\right)\right) & =\operatorname{dim}\left(W_{\mathbb{K}}\left(\boldsymbol{\omega}_{1} \omega_{2}\right)\right) \quad \text { and } \\
\operatorname{dim}\left(W_{\mathbb{}}\left(\boldsymbol{\omega}_{i}\right)\right) & =\operatorname{dim}\left(W_{\mathbb{F}}\left(\varpi_{i}\right)\right), \quad i=1,2 .
\end{aligned}
$$

On the other hand, it follows from Theorem 1.5.2(d) in characteristic zero that

$$
\operatorname{dim}\left(W_{\mathbb{K}}\left(\boldsymbol{\omega}_{1} \boldsymbol{\omega}_{2}\right)\right)=\operatorname{dim}\left(W_{\mathbb{K}}\left(\boldsymbol{\omega}_{1}\right)\right) \operatorname{dim}\left(W_{\mathbb{K}}\left(\boldsymbol{\omega}_{2}\right)\right) .
$$

Since (5.6.3) and (5.6.4) clearly imply (5.6.2), we are done.

5.7. Fusion products. We finish the paper with an application of Theorems 1.5 .2 and 5.5.1 related to the concept of fusion products originally introduced in the characteristic-zero setting. Namely, we deduce the positive characteristic counterpart of [Naoi 2012, Corollary B] (compare [Fourier and Littelmann 2007, Corollary A] for simply-laced $\mathfrak{g})$.

Let $V$ and $W$ be as in Theorem 5.5.1, set $\lambda=\operatorname{wt}(\boldsymbol{\omega})+\operatorname{wt}(\pi)$, and fix $v \in$ $(V \otimes W)_{\lambda} \backslash\{0\}$. Then Theorem 5.5.1 implies that $V \otimes W=U_{\mathbb{F}}(\tilde{\mathfrak{g}}) v$. In fact, as 
mentioned in Section 3.4, we actually have

$$
V \otimes W=U_{\mathbb{F}}\left(\mathfrak{n}^{-}[t]\right) v .
$$

Define the fusion product of $V$ and $W$, denoted $V * W$, as the $U_{\mathbb{F}}(\mathfrak{g}[t])$-module $\operatorname{gr}(V \otimes W)$ with the module structure determined by $v$ as described in the paragraph after Proposition 3.4.1. Evidently, if we have a collection $\omega_{1}, \ldots, \omega_{m}$ of relatively prime elements of $\mathscr{P}_{\mathbb{F}}^{+}$and, for each $j \in\{1, \ldots, m\}, V_{j}$ is a quotient of $W_{\mathbb{F}}\left(\boldsymbol{\omega}_{j}\right)$, we can define the fusion product $V_{1} * \cdots * V_{m}$ in a similar way.

Proposition 5.7.1. Let $\lambda \in P^{+}, m \in \mathbb{Z}_{>0}$ and $\omega_{j} \in \mathscr{P}_{\mathbb{F}}^{+}, j=1, \ldots, m$, be relatively prime and such that $\lambda=\sum_{j=1}^{m} \operatorname{wt}\left(\omega_{j}\right)$. Then

$$
W_{\mathbb{F}}^{c}(\lambda) \cong W_{\mathbb{F}}\left(\omega_{1}\right) * \cdots * W_{\mathbb{F}}\left(\boldsymbol{\omega}_{m}\right) .
$$

Proof. One easily checks that a vector in $\left(W_{\mathbb{F}}\left(\omega_{1}\right) * \cdots * W_{\mathbb{F}}\left(\boldsymbol{\omega}_{m}\right)\right)_{\lambda}$ satisfies the defining relations of $W_{\mathbb{F}}^{c}(\lambda)$ (compare the proof of (1.5.4) in Section 3.4), showing that $W_{\mathbb{F}}\left(\omega_{1}\right) * \cdots * W_{\mathbb{F}}\left(\omega_{m}\right)$ is a quotient of $W_{\mathbb{F}}^{c}(\lambda)$. On the other hand, setting $\omega=\prod_{j=1}^{m} \omega_{j}$, we have

$$
\begin{aligned}
& \operatorname{dim}\left(W_{\mathbb{F}}\left(\boldsymbol{\omega}_{1}\right) * \cdots * W_{\mathbb{F}}\left(\boldsymbol{\omega}_{m}\right)\right) \\
& \quad=\operatorname{dim}\left(W_{\mathbb{F}}\left(\boldsymbol{\omega}_{1}\right) \otimes \cdots \otimes W_{\mathbb{F}}\left(\boldsymbol{\omega}_{m}\right)\right)=\operatorname{dim}\left(W_{\mathbb{F}}(\boldsymbol{\omega})\right)=\operatorname{dim}\left(W_{\mathbb{F}}^{c}(\lambda)\right) .
\end{aligned}
$$

The following corollary, which is the characteristic-free version of [Naoi 2012, Corollary B], is now easily deduced.

Corollary 5.7.2. Let $m \in \mathbb{Z}_{>0}, \lambda_{j} \in P^{+}$and $a_{j} \in \mathbb{F}^{\times}, j=1, \ldots, m$, be such that $a_{i} \neq a_{j}$ for $i \neq j$. Then, for $\lambda=\sum_{j=1}^{m} \lambda_{j}, W_{\mathbb{F}}^{c}(\lambda) \cong W_{\mathbb{F}}\left(\boldsymbol{\omega}_{\lambda_{1}, a_{1}}\right) * \cdots * W_{\mathbb{F}}\left(\boldsymbol{\omega}_{\lambda_{m}, a_{m}}\right)$.

\section{References}

[Bianchi and Moura 2014] A. Bianchi and A. Moura, "Finite-dimensional representations of twisted hyper-loop algebras", Comm. Algebra 42:7 (2014), 3147-3182. MR 3178065 Zbl 06298126

[Carter 1972] R. W. Carter, Simple groups of Lie type, Pure and Applied Mathematics 28, Wiley, London, 1972. MR 53 \#10946 Zbl 0248.20015

[Chamberlin 2013] S. Chamberlin, "Integral bases for the universal enveloping algebras of map algebras", J. Algebra 377 (2013), 232-249. MR 3008904 Zbl 1293.17013

[Chari and Loktev 2006] V. Chari and S. Loktev, "Weyl, Demazure and fusion modules for the current algebra of $\mathfrak{s l}_{r+1}$ ", Adv. Math. 207:2 (2006), 928-960. MR 2008a:17029 Zbl 1161.17318

[Chari and Pressley 1986] V. Chari and A. Pressley, "New unitary representations of loop groups", Math. Ann. 275:1 (1986), 87-104. MR 88f:17029 Zbl 0603.17012

[Chari and Pressley 2001] V. Chari and A. Pressley, "Weyl modules for classical and quantum affine algebras”, Represent. Theory 5 (2001), 191-223. MR 2002g:17027 Zbl 0989.17019

[Chari and Venkatesh 2014] V. Chari and R. Venkatesh, "Demazure modules, Fusion products and $Q$-systems", Comm. Math. Phys. (online publication October 2014). arXiv 1305.2523 
[Chari et al. 2010] V. Chari, G. Fourier, and T. Khandai, "A categorical approach to Weyl modules", Transform. Groups 15:3 (2010), 517-549. MR 2011m:17014 Zbl 1245.17004

[Feigin and Loktev 2004] B. Feigin and S. Loktev, "Multi-dimensional Weyl modules and symmetric functions", Comm. Math. Phys. 251:3 (2004), 427-445. MR 2005m:17005 Zbl 1100.17005

[Fourier and Kus 2013] G. Fourier and D. Kus, "Demazure modules and Weyl modules: the twisted current case", Trans. Amer. Math. Soc. 365:11 (2013), 6037-6064. MR 3091275 Zbl 1287.17016 arXiv 1108.5960

[Fourier and Littelmann 2007] G. Fourier and P. Littelmann, "Weyl modules, Demazure modules, KR-modules, crystals, fusion products and limit constructions", Adv. Math. 211:2 (2007), 566-593. MR 2008k:17005 Zbl 1114.22010

[Fourier et al. 2012] G. Fourier, T. Khandai, D. Kus, and A. Savage, "Local Weyl modules for equivariant map algebras with free abelian group actions", J. Algebra 350 (2012), 386-404. MR 2859894 Zbl 1268.17022

[Fourier et al. 2014] G. Fourier, N. Manning, and A. Savage, "Global Weyl modules for equivariant map algebras", Int. Math. Res. Notes (online publication January 2014). arXiv 1303.4437

[Garland 1978] H. Garland, "The arithmetic theory of loop algebras", J. Algebra 53:2 (1978), 480551. MR 80a:17012 Zbl 0383.17012

[Humphreys 1978] J. E. Humphreys, Introduction to Lie algebras and representation theory, Graduate Texts in Mathematics 9, Springer, New York-Berlin, 1978. MR 81b:17007 Zbl 0447.17001

[Humphreys 1990] J. E. Humphreys, Reflection groups and Coxeter groups, Cambridge Studies in Advanced Mathematics 29, Cambridge University Press, 1990. MR 92h:20002 Zbl 0725.20028

[Jakelić and Moura 2007] D. Jakelić and A. Moura, "Finite-dimensional representations of hyper loop algebras", Pacific J. Math. 233:2 (2007), 371-402. MR 2009k:17014 Zbl 1211.17008

[Jakelić and Moura 2010] D. Jakelić and A. Moura, "Finite-dimensional representations of hyper loop algebras over non-algebraically closed fields", Algebr. Represent. Theory 13:3 (2010), 271-301. MR 2011i:17013 Zbl 1211.17007

[Jakelić and Moura 2014] D. Jakelić and A. Moura, "On Weyl modules for quantum and hyper loop algebras", pp. 99-134 in Recent advances in representation theory, quantum groups, algebraic geometry, and related topics, edited by P. N. Achar et al., Contemporary Mathematics 623, American Mathematical Society, Providence, RI, 2014.

[Jantzen 2003] J. C. Jantzen, Representations of algebraic groups, 2nd ed., Mathematical Surveys and Monographs 107, American Mathematical Society, Providence, RI, 2003. MR 2004h:20061 Zbl 1034.20041

[Joseph 2003] A. Joseph, "A decomposition theorem for Demazure crystals", J. Algebra 265:2 (2003), 562-578. MR 2004f:17035 Zbl 1100.17009

[Joseph 2006] A. Joseph, "Modules with a Demazure flag", pp. 131-169 in Studies in Lie theory, edited by J. Bernstein et al., Progress in Mathematics 243, Birkhäuser, Boston, 2006. MR 2007b: 17023 Zbl 1195.17010

[Littelmann 1998] P. Littelmann, "Contracting modules and standard monomial theory for symmetrizable Kac-Moody algebras”, J. Amer. Math. Soc. 11:3 (1998), 551-567. MR 99d:17027 Zbl 0915.20022

[Macedo 2013] T. Macedo, Characters and cohomology of modules for affine Kac-Moody algebras and generalizations, Ph.D. thesis, Universidade Estadual, Campinas, 2013, Available at http:// www.bibliotecadigital.unicamp.br/document/?code=000907347.

[Mathieu 1988] O. Mathieu, Formules de caractères pour les algèbres de Kac-Moody générales, Astérisque 159-160, Société Mathématique de France, Paris, 1988. MR 90d:17024 Zbl 0683.17010 
[Mathieu 1989] O. Mathieu, "Construction d'un groupe de Kac-Moody et applications", Compositio Math. 69:1 (1989), 37-60. MR 90f:17012 Zbl 0678.17012

[Mitzman 1985] D. Mitzman, Integral bases for affine Lie algebras and their universal enveloping algebras, Contemporary Mathematics 40, American Mathematical Society, Providence, RI, 1985. MR 86h:17022 Zbl 0572.17009

[Naoi 2012] K. Naoi, "Weyl modules, Demazure modules and finite crystals for non-simply laced type", Adv. Math. 229:2 (2012), 875-934. MR 2855081 Zbl 05994259

Received July 23, 2013.

ANGELO BIANCHI

Instituto DE CiênCIA E TECNOLOGia

Universidade Federal de São PaUlo

12231-280 SÃO JOSÉ DOS CAMPOS - SP

BRAZIL

acbianchi@unifesp.br

Tiago Macedo

Instituto de CiênCIA E TECNOLOGia

Universidade Federal de São PAUlo

12231-280 SÃO JOSÉ DOS CAMPOS - SP

BRAZIL

tmacedo@unifesp.br

ADRIANO MOURA

Instituto de MATEMÁticA, Estatística E ComputaÇÃo CientíficA

UNIVERSIDADE ESTADUAL DE CAMPINAS

CAIXA Postal 6065

13083-859 CAMPINAS - SP

BRAZIL

aamoura@ime.unicamp.br 


\title{
PACIFIC JOURNAL OF MATHEMATICS
}

\author{
msp.org/pjm
}

Founded in 1951 by E. F. Beckenbach (1906-1982) and F. Wolf (1904-1989)

\section{EDITORS}

Don Blasius (Managing Editor)

Department of Mathematics

University of California

Los Angeles, CA 90095-1555

blasius@math.ucla.edu

\author{
Paul Balmer \\ Department of Mathematics \\ University of California \\ Los Angeles, CA 90095-1555 \\ balmer@math.ucla.edu \\ Robert Finn \\ Department of Mathematics \\ Stanford University \\ Stanford, CA 94305-2125 \\ finn@math.stanford.edu \\ Sorin Popa \\ Department of Mathematics \\ University of California \\ Los Angeles, CA 90095-1555 \\ popa@math.ucla.edu
}

\author{
Vyjayanthi Chari \\ Department of Mathematics \\ University of California \\ Riverside, CA 92521-0135 \\ chari@math.ucr.edu \\ Kefeng Liu \\ Department of Mathematics \\ University of California \\ Los Angeles, CA 90095-1555 \\ liu@math.ucla.edu \\ Jie Qing \\ Department of Mathematics \\ University of California \\ Santa Cruz, CA 95064 \\ qing@ cats.ucsc.edu
}

\section{PRODUCTION}

Silvio Levy, Scientific Editor, production@msp.org

\section{SUPPORTING INSTITUTIONS}

ACADEMIA SINICA, TAIPEI

CALIFORNIA INST. OF TECHNOLOGY

INST. DE MATEMÁTICA PURA E APLICADA

KEIO UNIVERSITY

MATH. SCIENCES RESEARCH INSTITUTE

NEW MEXICO STATE UNIV.

OREGON STATE UNIV.

\author{
STANFORD UNIVERSITY \\ UNIV. OF BRITISH COLUMBIA \\ UNIV. OF CALIFORNIA, BERKELEY \\ UNIV. OF CALIFORNIA, DAVIS \\ UNIV. OF CALIFORNIA, LOS ANGELES \\ UNIV. OF CALIFORNIA, RIVERSIDE \\ UNIV. OF CALIFORNIA, SAN DIEGO \\ UNIV. OF CALIF., SANTA BARBARA
}

\author{
Daryl Cooper \\ Department of Mathematics \\ University of California \\ Santa Barbara, CA 93106-3080 \\ cooper@math.ucsb.edu \\ Jiang-Hua Lu \\ Department of Mathematics \\ The University of Hong Kong \\ Pokfulam Rd., Hong Kong \\ jhlu@maths.hku.hk \\ Paul Yang \\ Department of Mathematics \\ Princeton University \\ Princeton NJ 08544-1000 \\ yang@math.princeton.edu
}

These supporting institutions contribute to the cost of publication of this Journal, but they are not owners or publishers and have no responsibility for its contents or policies.

See inside back cover or msp.org/pjm for submission instructions.

The subscription price for 2015 is US \$420/year for the electronic version, and \$570/year for print and electronic.

Subscriptions, requests for back issues and changes of subscribers address should be sent to Pacific Journal of Mathematics, P.O. Box 4163, Berkeley, CA 94704-0163, U.S.A. The Pacific Journal of Mathematics is indexed by Mathematical Reviews, Zentralblatt MATH, PASCAL CNRS Index, Referativnyi Zhurnal, Current Mathematical Publications and Web of Knowledge (Science Citation Index).

The Pacific Journal of Mathematics (ISSN 0030-8730) at the University of California, c/o Department of Mathematics, 798 Evans Hall \#3840, Berkeley, CA 94720-3840, is published twelve times a year. Periodical rate postage paid at Berkeley, CA 94704, and additional mailing offices. POSTMASTER: send address changes to Pacific Journal of Mathematics, P.O. Box 4163, Berkeley, CA 94704-0163.

PJM peer review and production are managed by EditFLOW ${ }^{\circledR}$ from Mathematical Sciences Publishers.

\section{PUBLISHED BY}

\section{mathematical sciences publishers \\ nonprofit scientific publishing}

http://msp.org/

(C) 2015 Mathematical Sciences Publishers 


\section{PACIFIC JOURNAL OF MATHEMATICS}

Volume $274 \quad$ No. $2 \quad$ April 2015

On Demazure and local Weyl modules for affine hyperalgebras

Angelo Bianchi, Tiago Macedo and Adriano Moura

On curves and polygons with the equiangular chord property

305

TARik Aougab, Xidian Sun, Serge TabachniKov and

YUWEN WANG

The well-posedness of nonlinear Schrödinger equations in Triebel-type 325 spaces

SHAOLEI RU and JiECHENG CHEN

Hypersurfaces with constant curvature quotients in warped product manifolds

JIE WU and CHAO XIA

The first terms in the expansion of the Bergman kernel in higher degrees

MARTin PUCHOL and JiAlin ZHU

Determinant rank of $C^{*}$-algebras

Guinua Gong, HuAXIN Lin and Yifeng XUE

Motion by mixed volume preserving curvature functions near spheres

437

DAVID HARTLEY

Homomorphisms on infinite direct products of groups, rings and monoids

GEORGE M. BERGMAN

The virtual first Betti number of soluble groups

Martin R. BRIDSON and Dessislava H. KochloukOVA 